\title{
Using Magnetoencephalography to Monitor the Progression from Mild Cognitive Impairment to Alzheimer Disease: An Approach Based on Resting State Functional Connectivity.
}

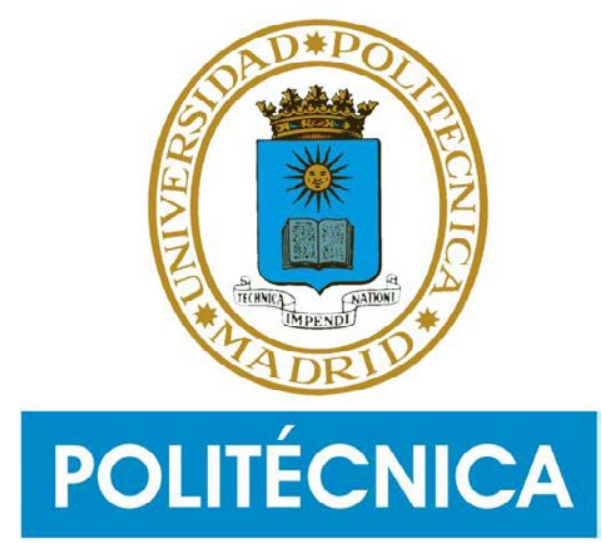

Sandra Angélica Pusil

Laboratorio de Neurociencia Cognitiva y Computacional

Centro de Tecnología Biomédica

Universidad Politécnica de Madrid

Supervised by:

Ernesto Pereda

Francisco Barceló

A thesis submitted for the degree of

Philosophiæ Doctor (PhD) in Biomedical Engineering 
That memory, the warder of the brain, Shall be a fume, and the receipt of reason...

Shakespeare. 


\section{Abstract}

Alzheimer's disease (AD) is a neurodegenerative disease, currently considered the most common type of dementia corresponding to $60-70 \%$ of the cases in the world population. This disease is clinically defined by a progressive loss of memory and other cognitive and functional abilities. Histologically, AD is characterized by the presence of amyloid plaques, neurofibrillary tangles and brain atrophy.

Recent studies have focused on early stages of the disease to generate diagnostic treatments that can prevent the progression of this disease. One of the most studied phases is Mild Cognitive Impairment (MCI). In this stage, the individual presents a slight but appreciable and measurable decrease in cognitive abilities, including memory, without completely affecting the development of the person's daily activities. These patients have a high risk of developing AD, especially those who have memory impairment. These first signs of decline can be measured through biomarkers such as the accumulation of beta-amyloid and p-tau in the cerebrospinal fluid (CSF), neuropsychological evaluation and the study of cerebral atrophy, especially of the hippocampus, by means of magnetic resonance imaging.

In this context, Magnetoencephalography (MEG), a promising neuroimaging technique, measures the post-synaptic potentials generated by billions of neurons, which can be used to study functional networks alterations in neuropathologies such as AD. Thus, functional connectivity (FC) offers an integrative vision, where brain functions are carried out through the communication and interaction of various brain regions. Additionally, effective connectivity (EC) defines the direction of the flow of information between brain regions, considering a simultaneous interaction of several neural elements to explicitly quantify the effect one element has on another. Finally, graph theory allows the characterization of the functional networks derived from a connectivity analysis. 
Therefore, the aim of this thesis is to study the functional connectivity alterations in patients with MCI that subsequently evolve to AD. Likewise, we also seek to associate these patterns to diverse biomarkers such as the accumulation of beta-amyloid and tau proteins as well as memory impairment and hippocampal atrophy. Finally, in order to study the disease, we propose a model that allows the prediction of which subjects with mild cognitive impairment will develop AD.

Chapter 3 presents healthy elderly subjects under scopolamine administration. Scopolamine is an anti-muscarinic that produces an anticholinergic effect and induces cognitive alterations (such as temporary memory loss) similar to those observed in AD. In this study, MEG brain patterns were analyzed through FC. In addition, graph theory was applied to characterize these networks. Thus, FC alterations were obtained, such as the decrease in synchronization in high frequency bands and a network organization similar to that found in $\mathrm{AD}$ patients.

Chapter 4 shows MCI patients from whom CSF was extracted to study the association between pathological accumulation of beta-amyloid and p-tau with changes in MEG FC patterns. Also, alterations in structural connectivity were studied and related to alterations in FC. Thus, it was found that patients with abnormal accumulation of beta-amyloid and p-tau in the CSF, showed a significant reduction and increase in FC affecting different brain regions including the anterior and posterior cingulate, the orbito-frontal cortex and the medial temporal areas in different frequency bands. In addition, a reduction in the connectivity of the posterior cingulate mediated by the p-tau protein was found; this was associated with the degeneration of the axonal integrity of the hippocampal cingulum. Finally, the biomarkers of the CSF and the scores obtained from the neuropsychological assessment predicted most of the alterations in FC.

Lastly, in Chapter 5, a longitudinal study of MCI patients was conducted. These patients were follow-up for three years and then split into two groups according to the final clinical outcome, as "stable" MCI (sMCI) and patients with "progressive" $\mathrm{MCI}$ (pMCI), the latter being those patients who during the three years converted to AD. Two MEG measurements were recorded at the beginning of the study and then 3 years later for each patient. FC and EC were studied between 
both groups as well as the association of neuropsychological assessment and hippocampal volumes with FC. In addition, with these variables, a model was proposed to classify the subjects in "sMCI" or "pMCI".

On the other hand, a network analysis was performed with the FC, studying the participation coefficient; this index highlights the nodes that are highly involved with other sub-networks in the brain. Also, the in- and out-degree was computed from the EC with the phase transfer entropy. Finally, to complement the network analysis, we studied the multiple participation coefficient, which is computed for a multiplex network consisting of four layers corresponding to the frequency bands analyzed. This analysis provides additional information about the regions that actively participate in the four layers of this multiplex network.

Patients who developed AD showed a disruption of FC in the theta and beta bands compared to those who remained stable. The alteration of this connectivity in the theta correlated with two neuropsychological tests usually related to memory: the immediate and delayed recall, also, with hippocampus volumes in theta and beta.

Changes in FC in theta and beta band along with the neuropsychological tests of inverse digits and the trail making test, both related to attentional and executive functions, predicted the conversion to AD with high accuracy.

Likewise, the FC in theta and beta band of the first MEG measurement together with the immediate recall and the direct digit test predicted the conversion to $\mathrm{AD}$, although not with a precision as high as the previous model.

The directed networks obtained from EC showed that pMCI presented a more disorganized and disconnected network in comparison to sMCI. The findings found here corroborate the theory of FC and EC disrupted networks in patients with $\mathrm{AD}$. In this chapter, MEG revealed itself as a promising tool to measure synaptic disruption in $\mathrm{AD}$ patients. Likewise, it is important to highlight the importance of combining different perspectives to support diagnosis and prediction using neuropsychological and anatomical variables such as hippocampal volumes, whose alterations represent the cognitive deficits and cerebral atrophy observed in AD. 
This thesis aims to provide a contribution to the study of AD and support MEG along with FC as tools with immense potential in the study of functional brain networks and their alterations in $\mathrm{MCI}$ and its progression to AD. 


\section{Resumen}

El estudio del cerebro es uno de los campos más interesantes y complejos de la ciencia actual. Tanto la investigación del cerebro sano como el estudio de neuropatologías de alta prevalencia mundial llenan las páginas de todas las revistas científicas del mundo. Dentro de las neuropatologías, el Alzheimer es una enfermedad neurodegenerativa, actualmente considerada como el tipo más común de demencia correspondiente al 60-70\% de los casos en la población mundial. Esta enfermedad esta clínicamente definida por una pérdida progresiva de memoria y otras habilidades cognitivas y funcionales. Histológicamente, el Alzheimer se caracteriza por la presencia de placas de amiloide, ovillos neurofibrilares y atrofia cerebral.

Muchos de los estudios científicos actuales se centran en etapas tempranas de la enfermedad para crear tratamientos diagnósticos que puedan prevenir el avance de esta enfermedad. Una de las fases más investigadas es el deterioro cognitivo leve. En esta etapa, el individuo presenta una disminución leve pero apreciable y medible de las capacidades cognitivas, entre ellas la memoria, sin afectar por completo el desarrollo de las actividades diarias de la persona. Estos pacientes tienen un alto riesgo de desarrollar Alzheimer, especialmente aquellos que presentan deterioro de la memoria. Estos primeros signos de deterioro pueden ser medidos a través de biomarcadores como la acumulación de beta-amiloide y tau en el líquido cefalorraquídeo, evaluación neuropsicológica y estudio de atrofia cerebral, especialmente de los hipocampos, por medio de resonancia magnética.

Dentro de este contexto, la Magnetoencefalografía aparece como una técnica de neuroimagen que permite estudiar los campos magnéticos cerebrales, específicamente la medición de potenciales post-sinápticos, la cual puede ser utilizada para estudiar alteraciones de redes funcionales en neuropatologías como el Alzheimer. De esta manera, la conectividad funcional ofrece una visión integradora, donde las funciones del cerebro se llevan a cabo mediante la comunicación e interacción de diversas regiones cerebrales. Adicionalmente, la 
conectividad efectiva permite conocer la dirección del flujo de información entre regiones cerebrales. Junto con las herramientas mencionadas anteriormente, la teoría de grafos permite la caracterización de las redes funcionales encontradas a partir de la conectividad.

Por lo tanto, el propósito de esta tesis es estudiar los patrones de las alteraciones de la conectividad funcional en pacientes con deterioro cognitivo leve que evolucionan posteriormente a Alzheimer. Así mismo, asociar estos patrones a diversos biomarcadores como la acumulación de las proteínas beta-amiloide y tau así como también, al deterioro de memoria y atrofia de hipocampo. Finalmente, de cara al estudio de la enfermedad y al diagnóstico de la misma proponer un modelo que permita predecir que sujetos con deterioro cognitivo leve evolucionaran a Alzheimer.

En el capítulo 3, se presenta un estudio en sujetos ancianos sanos a los cuales se les ha administrado escopolamina, un anti-muscarínico que produce un efecto anti-colinérgico e induce alteraciones cognitivas (como la perdida temporal de memoria) similares a las que se observan en Alzheimer. En este estudio, se analizaron los patrones cerebrales a través de la conectividad funcional. Además, para caracterizar estas redes encontradas se aplicó teoría de grafos. Así, se obtuvieron alteraciones en la conectividad funcional como el decremento de la sincronización en bandas de alta frecuencias y una organización de la red parecida a la que se ve en los pacientes con Alzheimer.

El capítulo 4, muestra una población de pacientes con deterioro cognitivo leve a los cuales se les extrajo líquido cefalorraquídeo para estudiar la asociación de la acumulación patológica de beta-amiloide y tau con los cambios en los patrones de conectividad funcional. También, se estudiaron alteraciones en la conectividad estructural y se relacionaron con las alteraciones en la conectividad funcional. Así, se encontró que los pacientes con valores anormales de proteínas beta-amiloide y tau en el líquido cefalorraquídeo mostraban una reducción e incremento de la conectividad funcional afectando a diferentes regiones cerebrales entre ellas el cingulado anterior y posterior, el córtex orbito-frontal y las áreas temporales mediales en diferentes bandas de frecuencias. Además, se encontró una reducción en la conectividad del cingulado posterior mediada por la proteína 
tau y esto fue asociado al deterioro de la integridad axonal del cingulum hipocampal. Finalmente, los biomarcadores del líquido cefalorraquídeo y los valores obtenidos de la evaluación neuropsicológica predijeron la mayoría de alteraciones de la conectividad funcional.

Por último, en el capítulo 5, se realizó un estudio longitudinal de pacientes con deterioro cognitivo leve. Estos pacientes fueron evaluados durante tres años y luego divididos en dos grupos de acuerdo al diagnóstico clínico final. Así se crearon dos grupos: pacientes con deterioro cognitivo leve "estables" y pacientes con deterioro cognitivo "progresivo", estos últimos son aquellos pacientes que durante esos tres años convirtieron a Alzheimer. Se registraron dos medidas de Magnetoencefalografía al comienzo del estudio y luego 3 años después para cada paciente. De estos dos grupos se estudió la conectividad funcional y efectiva. Así como la asociación de la valoración neuropsicológica y los volúmenes de hipocampo con la conectividad funcional. Además, con estas variables se propuso un modelo que permite realizar la clasificación de los sujetos en "estable" o "progresivo".

Por otro lado, se realizó un análisis de redes con el PLV, estudiando el coeficiente de participación, este índice permite conocer los nodos que están altamente involucrados con otras sub-redes en el cerebro. También, se calculó el in- y el out-degree a partir de la conectividad efectiva con la transferencia de entropía de fase. Finalmente, para complementar los análisis de red, se decidió estudiar el coeficiente de participación múltiple de una red multiplexada de cuatro capas correspondientes a las bandas de frecuencias analizadas. Este análisis puede dar información adicional de las regiones que participan activamente en las cuatro capas de esta red multiplexada.

Los pacientes que evolucionaron a Alzheimer mostraron una disrupción de la conectividad funcional en la banda theta y beta en comparación con los que permanecieron estables. La alteración de esta conectividad en theta correlacionó con dos test neuropsicológicos usualmente relacionados con la memoria: el recuerdo inmediato y demorado, también, con los volúmenes de hipocampo en theta y beta. 
Los cambios en la conectividad funcional en las bandas theta y beta junto con los test neuropsicológicos de dígitos inversos y el test del trazo ambos relacionados con funciones ejecutivas y de la atención, funciones deterioradas en el transcurso de la enfermedad de Alzheimer predijeron la conversión a Alzheimer.

Igualmente, la conectividad en theta y beta de la primera medida de Magnetoencefalografía junto con el recuerdo inmediato y el test de dígitos directos predijeron la conversión a Alzheimer, aunque no con una precisión tan alta como el modelo anterior.

Las redes con direccionalidad obtenidas de la conectividad efectiva mostraron que los conversores presentaban una red más desorganizada y desconectada en comparación a los estables.

Los hallazgos encontrados aquí corroboran la teoría de la disrupción de las redes funcional y efectiva en los pacientes con Alzheimer. En este capítulo, la Magnetoencefalografía representa una herramienta novedosa y prometedora de la disrupción sináptica en el diagnóstico y predicción de Alzheimer. Así mismo, es importante resaltar la importancia de combinar diferentes perspectivas para apoyar el diagnóstico y predicción utilizando variables neuropsicológicas y anatómicas como los volúmenes de hipocampo cuyas alteraciones representan los déficits cognitivos y atrofia cerebral presentes en Alzheimer.

Esta tesis pretende aportar un grano de arena en el estudio del cerebro y la enfermedad de Alzheimer y reforzar la Magnetoencefalografía junto con la conectividad funcional como herramientas novedosas con inmenso potencial en el estudio de las redes funcionales cerebrales y sus alteraciones, así como en la evolución del deterioro cognitivo leve a la enfermedad de Alzheimer. 


\section{Contents}

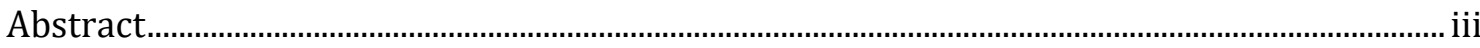

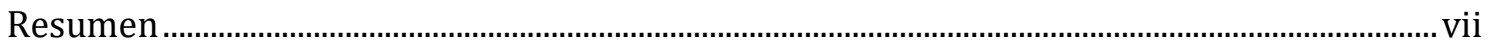

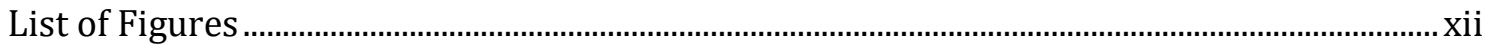

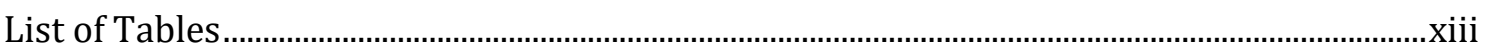

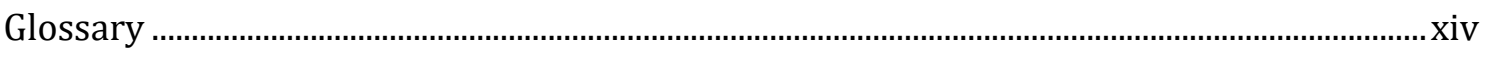

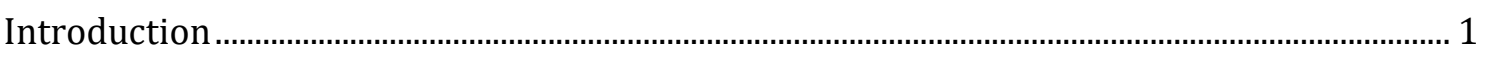

MEG and Functional Connectivity ........................................................................................

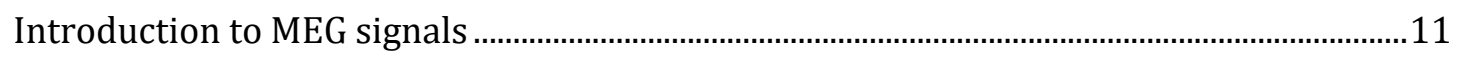

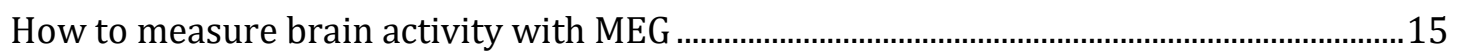

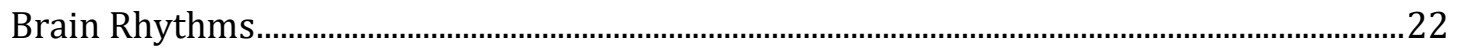

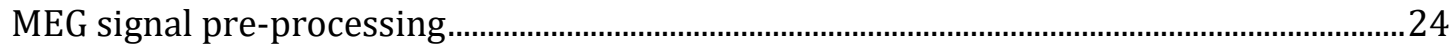

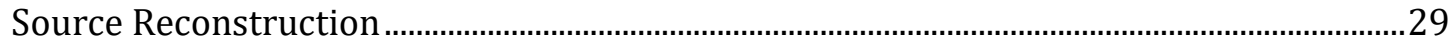

Functional Connectivity (FC)...............................................................................................38

Scopolamine effects on functional brain connectivity: a pharmacological model of

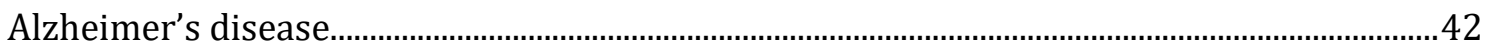

Materials and Methods ..............................................................................................................43

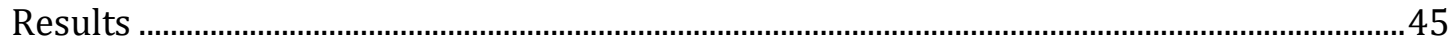

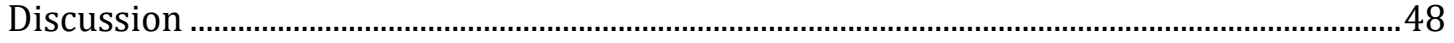

Network Disruption and Cerebrospinal Fluid Amyloid-Beta and Phospho-Tau Levels in

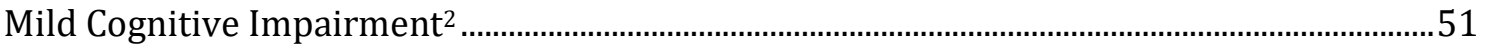

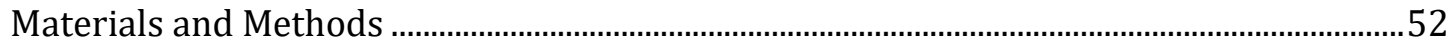

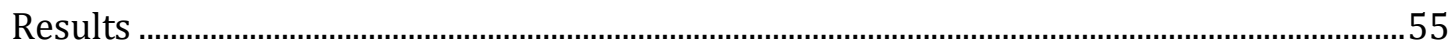

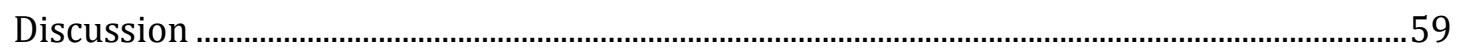

On the role of resting state MEG FC in longitudinal studies of MCI patients ............................63

Materials and Methods ................................................................................................................65

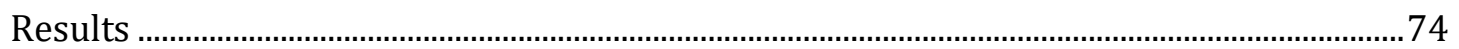

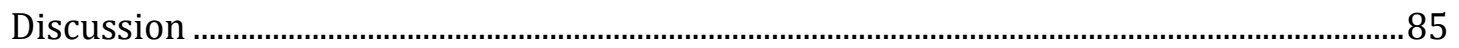

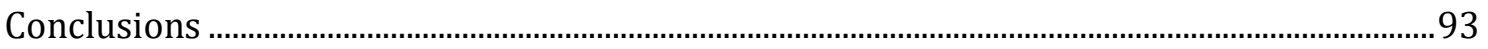

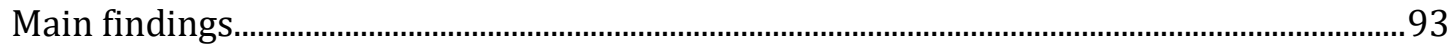

Limitations and methodological considerations.......................................................................96

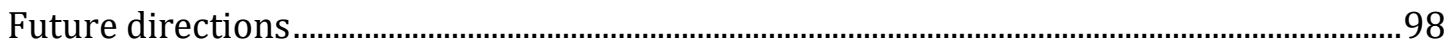

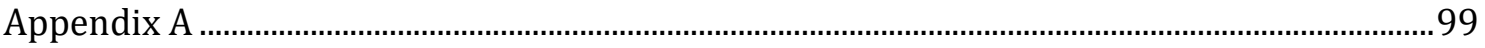

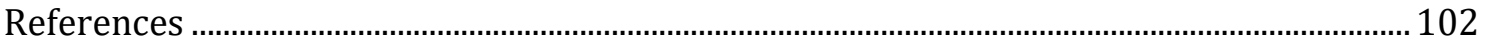




\section{List of Figures}

Figure 1. Clinical Disease Stages related with specific biomarkers ...................................... 3

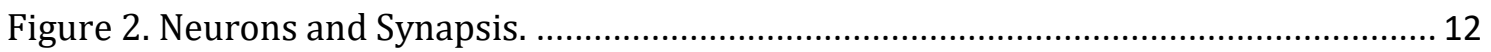

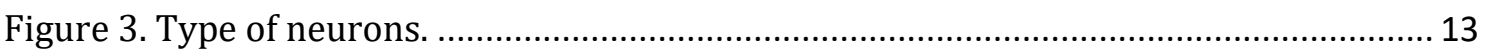

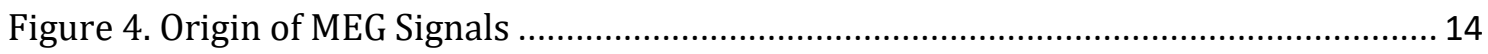

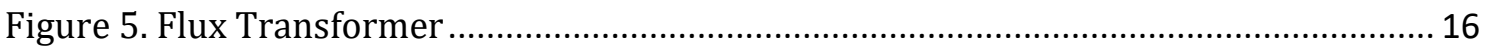

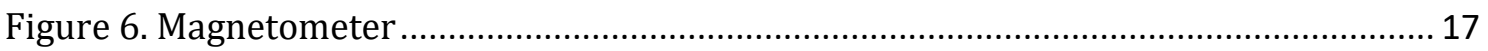

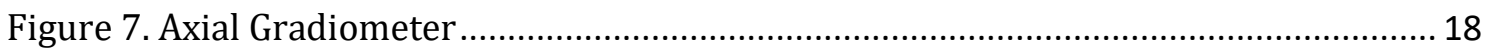

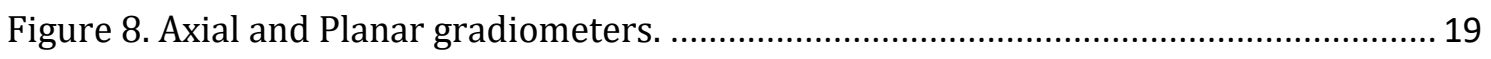

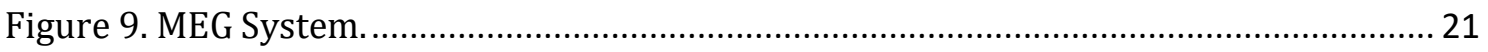

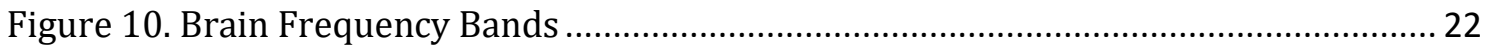

Figure 11. Maxfilter Source: Elekta-Neuromag Manual …................................................. 25

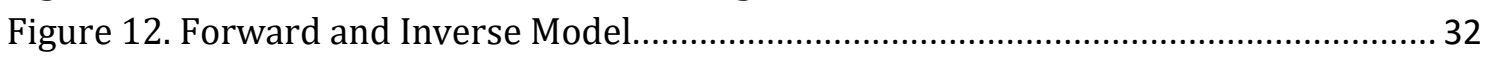

Figure 13. Radial component of the magnetic field in MEG .............................................. 33

Figure 14. Significant differences in PLV in Scopolamine vs Placebo................................... 46

Figure 15. Network parameters ( $C$ : clustering; $L$ : average shortest average path length).. 47

Figure 16. Pairs of regions showing significant functional connectivity abnormalities

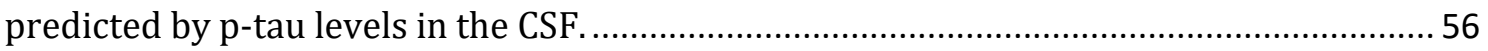

Figure 17. Pairs of regions showing significant functional connectivity abnormalities

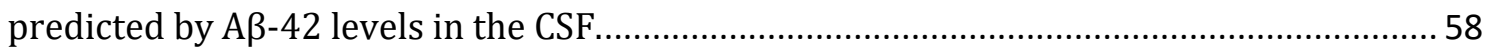

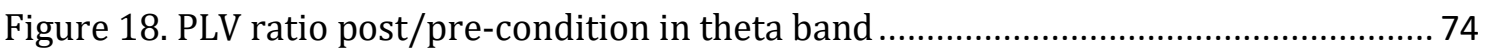

Figure 19. PLV ratio post/pre-condition in beta band ...................................................... 74

Figure 20. A. Correlations between the average PLV ratio of the significant clusters and the

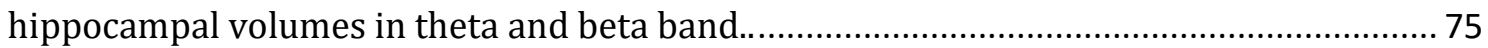

Figure 21. Correlations between PLV ratio and neuropsychological scores related with

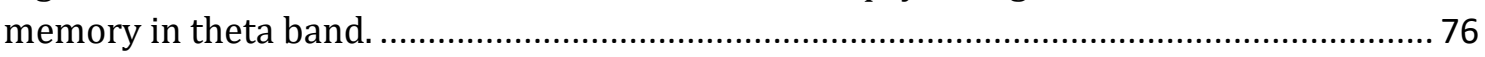

Figure 22. Evolution of each patient within each group in both conditions in theta band.. 77

Figure 23. Evolution of each patient within each group in both conditions in beta band... 77

Figure 24. In- and out-degree of PhTE connectivity in the pre-condition for pMCI............. 82

Figure 25. In- and out-degree of PhTE connectivity in the pre-condition for SMCI .............. 83

Figure 26. Multiple Participation Coefficient (MPC) for pre- and post-conditions ............... 84

Figure 27. PhTE for a wide range of analysis lags for different frequency bands .............. 101 


\section{List of Tables}

Table 1. Patients characteristics

Table 2. Phase synchronization and correlation values of cortical regions showing significantly different functional connectivity across biomarker groups 57

Table 3. Mean \pm SD values of the demographic and clinical characteristics of the sMCI and

pMCI patients 66

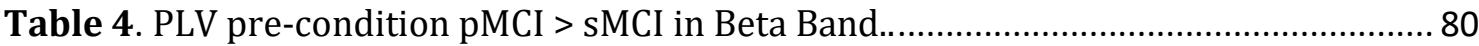

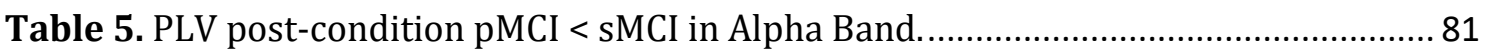

Table 6. Abbreviations for the 90 regions of the AAL atlas used in this study..................... 99 


\section{Glossary}

\begin{tabular}{ll} 
AAL & Automated Anatomic Labeling \\
AD & Alzheimer's disease \\
APOE & Apolipoprotein E \\
APP & Amyloid Precursor Protein \\
A $\beta$ & Amyloid Beta \\
BEM & Boundary Element Model \\
C & Clustering Coefficient \\
CBPT & Cluster Based Permutation Test \\
CSF & Cerebrospinal fluid \\
DMN & Default Mode Network \\
DTI & Diffusion Tensor Images \\
EC & Effective Connectivity \\
EEG & Electroencephalography \\
FAD & Familial Alzheimer's disease \\
FC & Functional Connectivity \\
ICA & Independent Component Analysis \\
L & Shortest Path Length \\
LCMV & Linearly Constrained Minimum Variance \\
MCI & Mild Cognitive Impairment \\
MEG & Magnetoencephalography \\
MMSE & Mini Mental State Examination \\
MNE & Minimum Norm Estimation \\
MPC & Multiple Participation Coefficient \\
MRI & Magnetic resonance imaging \\
MSR & Magnetically Shielded Room \\
PET & Positron emission tomography \\
PhTE & Phase Transfer Entropy \\
PLI & Phase Lag Index \\
PLV & Phase Locking Value \\
PS & Phase Synchronization \\
ROI & Region of Interest \\
SMD & Subjective Memory Decline \\
SPECT & Single Photon Emission Computed Tomography \\
SQUIDS & Superconducting Quantum Interference Devices \\
SSP & Signal Space Projection \\
SSS & Signal Space Separation \\
tSSS & Temporal Signal Space Separation \\
& \\
\hline &
\end{tabular}




\section{Chapter 1}

\section{Introduction}

Dementia is one of the most important problems of public health worldwide. This is partly due to the increase in life expectancy in developed countries and the exponential growth of the disorder with advancing age. Alzheimer's disease (AD) is the most common cause of dementia and may contribute to $60-70 \%$ of cases ${ }^{1}$. Worldwide, 46.8 million people are believed to be living with AD or other dementias $^{2}$. By 2030, if no treatment advances are made, we will see an increase to approximately 74.7 million. By 2050, rates could exceed 131.5 million. Every 3.2 seconds, a new case of dementia occurs somewhere in the world ${ }^{3}$. Particularly, in Spain only, there would be around 1.5 millions of people affected by this disease, with a prevalence of $7 \%$ for elderly people with more than 65 years 4,5 .

$\mathrm{AD}$ is a neurodegenerative disease, meaning it causes the degeneration, or loss, of neurons in the brain, particularly in the cortex. AD was first described in 1906 by Alois Alzheimer when he first studied a weird case of a patient named Auguste Deter $^{6}$, with atypical symptoms including severe behavioral changes, memory loss, cognitive deficits, and psychiatric symptoms, associated with macroscopic and microscopic brain lesions, in particular neuritic amyloid plaques, neurofibrillary tangles and brain atrophy. But about 70 years passed before the disease was recognized as a common cause of dementia and a major cause of death ${ }^{7}$.

AD causes disruptions in memory, cognition, personality, and other functions that eventually lead to complete brain failure and death. $\mathrm{AD}$ is characterized histologically by the presence of neurofibrillary tangles and neuritic amyloid plaques. These microscopic changes are accompanied by progressive brain atrophy ${ }^{8}$. Amyloid plaques are insoluble deposits of a peptide called amyloid beta $(A \beta)$. They are formed when the Amyloid Precursor Protein (APP) is sequentially cleaved by two enzymes: $\beta$-Secretase and $\gamma$-Secretase. A $\beta$ tends to unfold and become sticky, eventually clumping together to form soluble oligomers. Some of these aggregate into large insoluble fibrils that deposit in the brain as extracellular plaques. The oligomers come in several forms, or species. It is not known exactly which species are toxic, but research shows that they weaken the communication 
and plasticity at synapses. This could likely represent a key element to stop the brain from forming or retrieving memories ${ }^{9}$.

Another key feature of AD is neuro-degeneration. Neuronal death and damage is triggered by $A \beta$, but some of $A \beta$ 's effects seem to be mediated by another protein seen in the brain of AD patients: Tau protein, a component of tangles. In a healthy neuron, molecules are carried along the axon on a series of tracks made of microtubules and stabilized by Tau. But in AD, Tau is modified causing a dissociation from the microtubules, adopt an abnormal shape and move from the axon to the cell body. Like $A \beta$, Tau comes in a variety of forms and it is still is unknown which ones contribute to $\mathrm{AD}$. Additionally, like $\mathrm{A} \beta$, these forms either remain soluble, or stick together and deposit as the tangles present in the disease ${ }^{9}$.

Another problem seeing in animal's models is that misfolded Tau proteins can spread, across synapses, into healthy neurons. There, they make healthy Tau proteins start to unfold as well, spreading pathology across the brain ${ }^{10}$. This spreading starts in the hippocampus, the part of the brain where memories are first formed. Over many years, the plaques and tangles slowly destroyed the hippocampus, making it difficult for the hippocampus to be involved in new memory formation. Eventually, more plaques and tangles spread into different areas of the brain killing cells and compromising function in the affected brain areas. The pattern of spreading through different brain regions matches the changes in symptoms from early to late stages of $\mathrm{AD}^{10}$.

Due to the complexity of AD pathophysiology, this disease has brought much attention among neuroscientists and has become a significant area of research. Although research has unraveled many aspects of $\mathrm{AD}^{11}$, there are many intriguing issues yet to be discovered about the neurophysiological mechanisms underlying the disease. In this way, the earliest stages of the disease, before symptoms and clinical signs have reached the point at which a diagnosis of clinically probable AD can be made, offer a new approach to study and to identify first neurophysiological signs of AD. Thus, an early diagnosis can help to slow the progression of AD. This is the reason why many studies have focused in the early stages of $A D^{12-15}$. Such investigations may support the development or identification of strategies as well as more specific disease management approaches. 
It is known that the histopathological features or established biological markers of $\mathrm{AD}$ appear prior to the development of clinical symptoms of the disease. Currently, researchers use the term "pre-clinical AD" to refer to the full spectrum from completely asymptomatic individuals with biomarker evidence of $\mathrm{AD}$ to individuals manifesting subtle cognitive decline but who do not yet meet accepted clinical criteria for mild cognitive impairment (MCI). The pre-clinical AD is a newly defined stage of the disease reflecting current evidence that measurable biomarker changes in the brain may occur years before symptoms can be detected 16,17. A biomarker is an abnormality that can be measured to accurately and reliably indicate the presence of disease. The strongest biomarkers candidates for the pre-clinical stage, MCI and AD include brain imaging studies using magnetic resonance imaging (MRI) or positron emission tomography (PET), and the measurements of proteins levels (specifically $A \beta$ and Tau) in cerebrospinal fluid (CSF) ${ }^{18,19}$. Figure 1 sketches the evolution from early to late stages of $\mathrm{AD}$ and the possible sensitivity of the biomarkers in each stage related to the impairment.

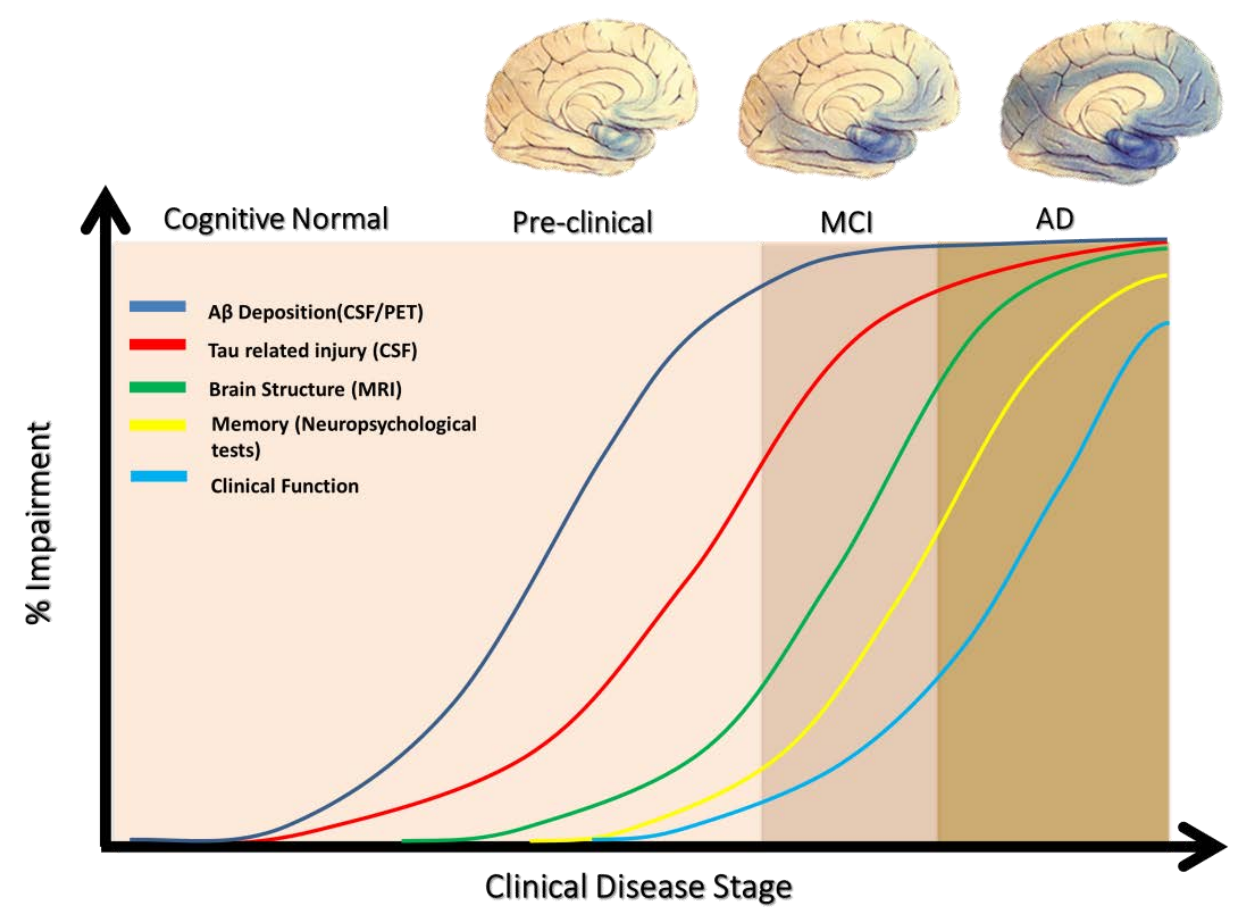

Figure 1. Clinical Disease Stages related with specific biomarkers 
While a quarter of AD patients have a strong family history of the disease, only $1 \%$ directly inherit a gene mutation that causes early-onset $\mathrm{AD}$, also known as familial $\mathrm{AD}(\mathrm{FAD})^{20}$. Early-onset $\mathrm{FAD}$ is caused by a number of different singlegene mutations on chromosomes 21, 14, and 1. Each of these mutations plays a role in the breakdown of APP and this breakdown is part of a process that generates harmful forms of amyloid plaques ${ }^{20}$.

Another gene called APOE influences the risk for the more common lateonset type of AD. There are three types of the APOE gene, called alleles: APOE2, E3 and E4. The E2 allele is the most uncommon form of APOE. There is evidence that carrying even one allele appears to reduce the risk of developing AD by up to $40 \%$. APOE3 is the most common allele and does not seem to influence the risk of the disease. The APOE4 allele, present in approximately $10-15 \%$ of people, increases the risk for $\mathrm{AD}$ and lowers the age of onset ${ }^{21,22}$.

Another interesting phase of the pre-clinical AD stage that has drawn attention over the last few years is Subjective Memory Decline (SMD) ${ }^{23}$. Older people reporting complaints of SMD but without evidence of objective cognitive decline on standard neuropsychological tests are increasingly considered at increased risk for AD. The first signs of cognitive decline among older adults are often memory-related. Forgetting a name, misplacing objects, or not remembering previous conversations becomes more common over the age of $50^{24}$, sometimes leading to concerns that this forgetfulness is more than just normal aging and may be related to early warning signs of possible AD.

Among all possible stages of $\mathrm{AD}, \mathrm{MCI}$ is probably one of the most studied because patients already exhibit clinical symptoms and the presence of all the neurophysiological and neuropsychological alterations can be measured through the aforementioned available biomarkers. Thus, MCI causes a slight but noticeable and measurable decline in cognitive abilities, including memory and cognitive skills, without complete disrupting the development of the person's daily activities. A subject with MCI is at a high risk of developing AD, especially those with memory impairment, although other domains can be affected in these patients as well as executive functions, language and attention; hereby MCI is sometimes considered as an early stage of AD. MCI is often measured with neuropsychological tests and 
currently the use of biomarkers has been incorporated to evaluate brain changes using MRI or PET and in CSF to measure the accumulation of $A \beta$ and Tau proteins. MCI patients who present pathologic accumulation of $\mathrm{A} \beta$ (around $60-70 \%$ of cases for subjects over 70 years old) ${ }^{25}$ are considered to be at an antecedent stage of $\mathrm{AD}^{26,27}$. Actually, MCI represents a 3-19\% of the population over 65 years old. It has been considered the focus of study of many clinical investigations and pharmacological interventions ${ }^{28}$ because of the annual conversion rate to AD that in MCI population is $10-15 \%$, reaching $80 \%$ over 6 years ${ }^{29}$.

Therefore, the study of MCI could give us some insight into the early stages of $\mathrm{AD}$, thus expanding the knowledge about how the disease develops. Since MCI patients present both an intermediate cognitive status between normal aging and the disease and high conversion rates to $\mathrm{AD}, \mathrm{MCI}$ may constitute an appropriate population to study the neurophysiological changes through the progression to AD. Since the early detection and prediction of the conversion to AD is an important issue nowadays, it is necessary to assess the anatomopathological alterations of AD through other perspectives including a neurophysiological viewpoint using techniques such as magnetoencephalography (MEG).

Additionally, in recent years, several studies have reported early synapsis dysfunction in early AD stages ${ }^{30-34}$. In this way, MEG is a neurophysiological tool to study synaptic dysfunction and disruption of connectivity because this technique provides a direct measure of neuronal field potentials, specifically the measure of post-synaptic potential that can be used to assess the organization of brain functional architecture.

MEG is a non-invasive neuro-imaging technique for investigating human brain activity. It allows measuring the neuronal electric activity on a millisecondby-millisecond basis and, in combination with structural MRI, locating the brain activity in the cortex. MEG is a direct measure of brain function, unlike techniques such as functional MRI, PET and single photon emission computed tomography (SPECT) that are secondary measures of brain function reflecting brain metabolism. MEG is complementary to other modalities and the information provided by each modality adds to the full picture. 
During the last decade, MEG has been widely used to study brain diseases like AD. The study of MCI and AD with MEG has been focused on the resting-state brain ${ }^{35-37}$. Basically, MEG brain patterns activations studied in AD suggest a slowing in the $\mathrm{AD}$ brain starting from posterior to anterior regions ${ }^{38-41}$, which are reflected in the slowing of the frequency power spectrum. This is characterized by an increase in power in slow frequency bands like delta $(2-4 \mathrm{~Hz})$ and theta $(4-8 \mathrm{~Hz})$, and a decrease in the fast frequency bands such as alpha $(8-12 \mathrm{~Hz})$, beta $(12-30 \mathrm{~Hz})$ and gamma $(30-45 \mathrm{~Hz})$. One of the reasons attributed to this phenomenon is the cholinergic theory, which will be explained later in this section.

In recent years, methods used to study the concepts of Functional Connectivity (FC) and Effective Connectivity (EC) have been widely applied by neuroscientists as tools in combination with MEG to characterize brain connectivity patterns in the healthy brain and in neurological diseases such as AD. FC is defined as the statistical dependence between two time series, while EC describes the causal influences that a specific time series exert over another. If two time series show synchronized patterns of activity they are likely to be functionally connected, but this should not necessarily implied a causal relationship.

One of the main finding in $\mathrm{AD}$ studies with $\mathrm{FC}$ is the decrease in connectivity in fast frequency bands, specifically in alpha ${ }^{42,43}$, although increased synchronization has also been observed in $\mathrm{AD}$ involving posterior brain regions $^{44,45}$. Also in MCI patients, reductions in FC between regions of the default mode network (DMN) have been reported. Additionally, some FC resting-state studies comparing different MCI groups have also detected a hypersynchronization pattern in fast frequency bands (alpha and beta) in those MCI subjects who progressed to $\mathrm{AD}^{46}$.

In this context, this thesis was born motivated by a desire to improve our understanding of $\mathrm{AD}$ brain connectivity patterns focusing in the progression of the disease starting from MCI to AD conversion using MEG and their relationship with cognition.

Although AD has many functional and structural alterations in the cerebral cortex, the most prominent abnormality lies in hippocampal damage, which leads to memory impairment. Thus, our first approach in this thesis was to study 
functional connectivity in healthy individuals with temporally memory loss induced by an anticholinergic medication to investigate the idea of a pharmacological model associated with memory impairment. In this way, the cholinergic theory of AD was studied.

Over 20 years has passed since the proposal of the cholinergic hypothesis, which is one of the oldest theories related to the cause of AD. This theory stablishes that $\mathrm{AD}$ begins as a deficit with the acetylcholine levels in the brain. It has been found that there is a 40\%-70\% loss of cholinergic neurons in the brain of an $\mathrm{AD}$ patient. Studies suggest that this happens because there is a shortage of two necessary enzymes. One enzyme is involved in the synthesis of acetylcholine, whereas the other is involved in the decomposition of this neurotransmitter after its message has been sent. Having too little of these enzymes will cause the neuron to stop working normally ${ }^{47}$.

Pathways of cholinergic neurons project widely throughout the brain. These pathways are damaged when these neurons undergo degenerative changes during the development of the disease. Consequently, this cholinergic cell loss could interfere with the ability of these cells to provide sufficient transmitter release for normal function. As a result, disruption to cholinergic function will produce specific brain regions alterations, such as hippocampal degeneration ${ }^{47}$, causing memory deficits typical of AD.

In our first study (Chapter 3), we used scopolamine, a muscarinic cholinergic receptor antagonist that produces a blockage of the activity of the muscarinic acetylcholine receptor. The administration of scopolamine in healthy individuals temporally impairs learning and memory, and influences the appearance of electrophysiological changes, including FC abnormalities, which may resemble those observed in $\mathrm{AD}^{48}$.

Since the first reports of a central cholinergic deficit associated to $\mathrm{AD}$, the connection has been made between the cognitive and memory deficits associated with this disease and the reversible amnestic effects induced by centrally acting muscarinic cholinergic antagonists like the scopolamine. Indeed, blockade of central muscarinic receptors could induce a pattern of cognitive impairment even in young subjects similar of that observed in the old subjects, or in individuals with 
AD. For many years, the amnestic action produced in animals by the administration of centrally acting muscarinic cholinergic antagonists, particularly scopolamine, has been a widely used model for the characterization of potential cognition-enhancing drugs ${ }^{49}$.

Next in Chapter 4, knowing that the definitive diagnosis of $\mathrm{AD}$ is anatomopathological, based on the presence of the deposits of abnormal $A \beta$ in the brain and the neurodegeneration caused by Tau protein, we explore the neurophysiological alterations related with the neuropathology of AD. Thus, we studied FC changes with MEG in MCI patients at risk of developing AD measuring the CSF levels of $A \beta$ and Tau and their cognitive status ${ }^{50}$. In this study, we use MEG to determine bio-magnetic patterns of resting-state FC associated with abnormal CSF levels of p-tau and A 342 in patients with MCI. We also aimed to determine an association between these patterns and structural connectivity abnormalities studied with MRI and the convergent impact of neuropsychological scores and CSF variables on network disorganization.

The likelihood that MCI is due to AD is strongly influenced by biomarkers. Accordingly, patients with MCI who progress to AD will likely show positive biomarkers of both $A \beta$ deposition and tau-related neuronal injury ${ }^{51}$. Low levels of CSF $A \beta$ peptides indicate increased $A \beta$ deposition in the brain and high levels of CSF tau protein derived from damaged neuronal microtubules are considered reliable biological markers of $\mathrm{AD}$ and predictors of $\mathrm{MCI}$ converters ${ }^{52}$. In particular, the phosphorylated form of Tau or "phospho-tau" (p-tau) appears to provide a high specificity in the diagnosis of $\mathrm{AD}$ compared with total Tau ${ }^{53}$. Increasing evidence indicates that synaptic dysfunction is a core deficit in $\mathrm{AD}$, preceding hallmark pathological abnormalities ${ }^{54,55}$. Soluble $A \beta$ oligomers and Tau fibrillar lesions affect synaptic plasticity and cause synaptic loss, leading to impairment of neural networks involved in memory and cognition. For this reason, MCI and AD are best characterized as a disruption of functional and structural integration of neural systems rather than as regionally localized abnormalities ${ }^{46,56-58}$.

Then, after studying the abnormal accumulation of the $A \beta$ and Tau proteins and how they affect brain connectivity patterns in MCI patients, in Chapter 5 we present the results from a longitudinal study of MCI patients and their progression 
to $\mathrm{AD}$ through the alteration of the cortical networks based on FC and EC. In this study, we aimed to analyze the progression to $\mathrm{AD}$ and determine the relationship of the FC, cognitive status and structural properties of MEG neural substrates of MCI patients who have converted to AD.

The early identification of MCI patients who will probably convert to AD is essential to initiate an early treatment to slow down the devastating effects of this neurodegenerative disorder. In this sense, MEG, FC and EC may provide relevant information in the progression of AD. In a recent MEG study performed by our group, pMCI subjects showed an increase in connectivity in alpha band between the right anterior cingulate and temporo-occipital regions than sMCI subjects. Moreover, this hypersynchronization was related with a worse cognitive status and a higher atrophy of temporal lobe structures ${ }^{46}$.

To the best of our knowledge, there is a remarkable amount of prospective studies focus on the progression from MCI to $\mathrm{AD}$, but there is a scarcity of longitudinal investigations that have used repeated measurements of the subjects over time, and none made with MEG.

Finally, this thesis will help to reinforce the use of MEG in combination with $\mathrm{MRI}$ and FC as an important tool to evaluate $\mathrm{AD}$ progression. 


\section{Chapter 2}

\section{MEG and Functional Connectivity}

Magnetoencephalography (MEG) is a non-invasive neuroimaging technique that measures weak magnetic fields generated by electric currents in the brain. It was pioneered by Cohen, who measured these current with a single channel instrument for the first time in $1968^{59}$. MEG is used to study brain dynamics and temporal changes in regional brain activation patterns and provides an excellent resolution of the timing of neuronal activity. Compared to other techniques such as Electroencephalography (EEG), MEG provides higher spatial resolution and is, therefore, more often used in brain mapping where the highest achievable resolution is needed (10-15 $\mathrm{mm})$. In addition, MEG is usually combined with MRI to get a good structural perspective.

Back in the 70's and 80's, researchers demonstrated the feasibility of using MEG to properly measuring brain activity. Starting with recordings of evoked responses to visual ${ }^{60}$, tactile $^{61}$ and auditory ${ }^{62,63}$ stimuli proving that the localization of these evoked fields came from the sensory-specific cortices. MEG was widely used to identify the cortical sources of various evoked and eventrelated potentials although EEG, due to its widespread availability, was more frequently used by researchers and physicians. Even so, MEG started to demonstrate its advantages. MEG studies at this time successfully differentiated between responses generated in the primary and secondary somatosensory cortices based on response timing, locations and directions of source current ${ }^{64}$. But it was not until the late 90's that MEG consolidated its position as a genuine brainmapping tool, with focus on activation sequences, supported by increasingly accurate visualization on spatially aligned anatomical MRI. The measurement times were markedly shortened and the quality of mapping was drastically improved as data were acquired simultaneously from the whole cortex. As a major step forward, whole-scalp MEG systems finally opened the path for studies on high-level cognition, such as language processing 65 or characterization of rhythmic 
activity throughout the cortex ${ }^{66}$. In addition, studies of brain rhythms were developed, focusing on frequencies from 5 to $40 \mathrm{~Hz}^{66}$, the Rolandic 20-Hz rhythm ${ }^{67}$, and gamma activity 68 , among others ${ }^{69,70}$. MEG research has expanded since then, including cognition, source reconstruction, functional and effective connectivity, training and development studies.

In this section, I will explain from MEG basis to MEG signal processing focusing on source reconstruction methods and functional connectivity (FC) analysis.

\section{Introduction to MEG signals}

The brain is the most complex organ in our body. It is what it is because of the structural and functional properties of interconnected neurons. Every minute of every day, the neurons in our brains send and receive signals that influence everything from the memories we form to the emotions we feel. Our brain has around 100 billion of neurons and each neuron can be connected to up to 10,000 other neurons. Because of their beauty and complexity, Ramon y Cajal called them butterflies of the soul ${ }^{71}$.

The neuron is the basic working unit of the brain, a specialized cell designed to transmit information to other nerve cells, muscle, or gland cells. The neurons are composed of three different parts: the soma, dendrites and a single axon (Figure 2). The soma is the part that contains the cell nucleus and cytoplasm. The dendrites are long, feathery and treelike filaments attached to the cell body. The dendrite is the primary site for receiving and integrating information from other neurons. Synapses are the contact points where one neuron communicates with another. The dendrites are covered with synapses formed by the ends of axons from other neurons. Finally, the axon extends from the cell body and often gives rise to many smaller branches before ending at nerve terminals which may be thousands of times the length of the soma. 


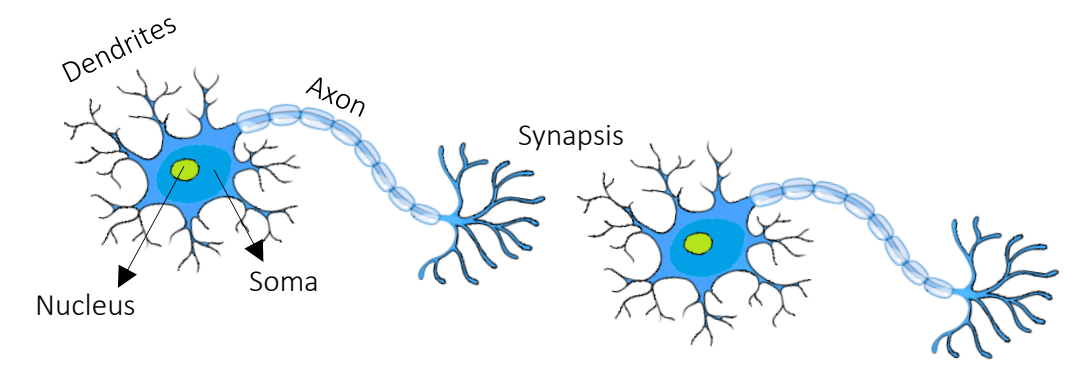

Figure 2. Neurons and Synapsis.

Source: https://askabiologist.asu.edu/anatomia-de-la-neurona

The neurons have the same genetic information, the same structural elements and perform the same basic functions as any other cell of the body. Although many cells of our body generate electric signals, only the neurons have the capacity to use this to communicate with them, because they transform these signals in their membranes so that they can be transmitted to other neurons and to another types of cells ${ }^{72}$.

Every neuron maintains a voltage gradient across its membrane, due to metabolically-driven differences in ions of sodium, potassium, chloride, and calcium within the cell, each of which has a different charge. If the voltage changes significantly, an electrochemical pulse called an action potential is generated ${ }^{73}$.

This pulse travels rapidly along the axon and is transferred across a specialized connection known as a synapse to a neighboring neuron, which receives it through its feathery dendrites. A synapse is a complex membrane gap used to transmit signals between cells, and this transfer is therefore known as a synaptic connection. Although axon-dendrite synaptic connections are the norm, other variations like dendrite-dendrite, axon-axon and dendrite-axon are also possible. A typical neuron fires 5 to 50 times per second ${ }^{73}$.

The neurons have a wide variety of sizes and shapes. According to their shape, scientists classified them as multipolar, unipolar, bipolar and pseudo-

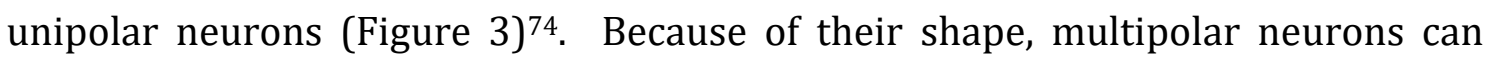
integrate a huge amount of information and connect to a great variety of neurons in the brain. In this sense, these neurons stand out for being the most abundant 
within the nervous central system. Among the multipolar neurons there exist several types including the motor, Purkinje, and the pyramidal neurons.

Pyramidal neurons (Figure 3d) are named after the shape of their cell body, which is similar to a pyramid. They have one axon, a large apical dendrite, multiple basal dendrites and dendritic spines. Pyramidal neurons, also called pyramidal cells, are found in different brain areas, such as the cerebral cortex, hippocampus, and amygdala. They were discovered and studied by Ramón y Cajal and since then, the different studies on the pyramidal neurons have focused in many different aspects ranging from neuronal plasticity to cognition ${ }^{75}$. Importantly, the pyramidal neurons, because of their shape and alignment (perpendicular to the cortex) effectively generate intracellular currents perpendicular to the cortical surface.

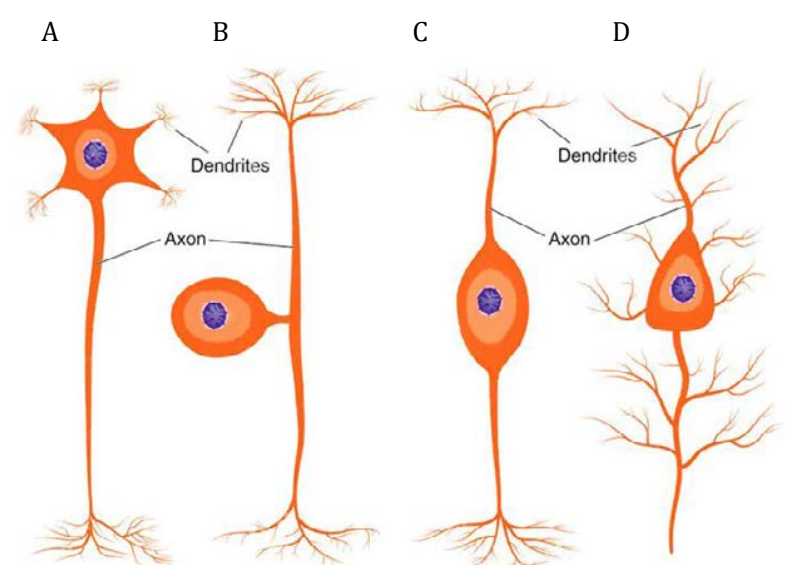

Figure 3. Type of neurons: A. Multipolar Neuron B. Unipolar Neuron C. Bipolar Neuron D. Pyramidal Neuron.

Source: http://understandingcontext.com/2012/06/varieties-nerve-cells/

As mentioned above, signal neurotransmission occurs by chemical processes that result in electrical currents spreading from the presynaptic to the postsynaptic neuron. Taking a closer look at this electrical phenomena, one can see that the arrival of the presynaptic action potential results in a release of neurotransmitters into the synaptic cleft ${ }^{76}$. These neurotransmitters, such as the glutamate, open the receptors of the postsynaptic neurons letting in charged ions that depolarize the postsynaptic membrane, thus setting in motion the postsynaptic potential ${ }^{72}$. This is what we can record outside of the brain rather than the presynaptic potential that caused it because the current of a single neuron is too small to be picked up outside of the brain; thus, MEG relies on the currents of 
many neurons summing up. Around $50 \times 10^{3}$ synchronously firing neurons may be necessary to produce a signal to be detected with $\mathrm{MEG}^{72}$. However, the presynaptic action potential is very short-lived, of the order of a few milliseconds. Furthermore, it is biphasic, which means it has both a positive and a negative deflection, and the signals that are not perfectly synchronized will somehow cancel each other out. Postsynaptic potentials, however, are much longer-lived as well as monophasic, this allows that their deflections add up easily in order to have a bigger chance of being measurable with MEG.

Our interest is then on the postsynaptic currents in the cortical pyramidal neurons that are the main primary currents giving rise to measurable MEG signals. These signals are highly sensitive to currents tangential to the skull, originating in the cortical sulci (Figure ${ }^{47}$ ). This is an advantage, as about two-thirds of the cerebral cortex is located within fissures (including all primary sensory cortices) that are difficult places to reach even with intracranial recordings. MEG fields reflect the primary neuronal currents directly, with minimum distortion from different layers (brain tissue, skull, and scalp) with different electric conductivities.

These currents in the pyramidal neurons of the cortex will generate coherent magnetic fields when activated with a certain degree of synchrony. We may say that these neurons behave as current dipoles, the activity of which can be detected by MEG sensors placed at a small distance from the skull78.

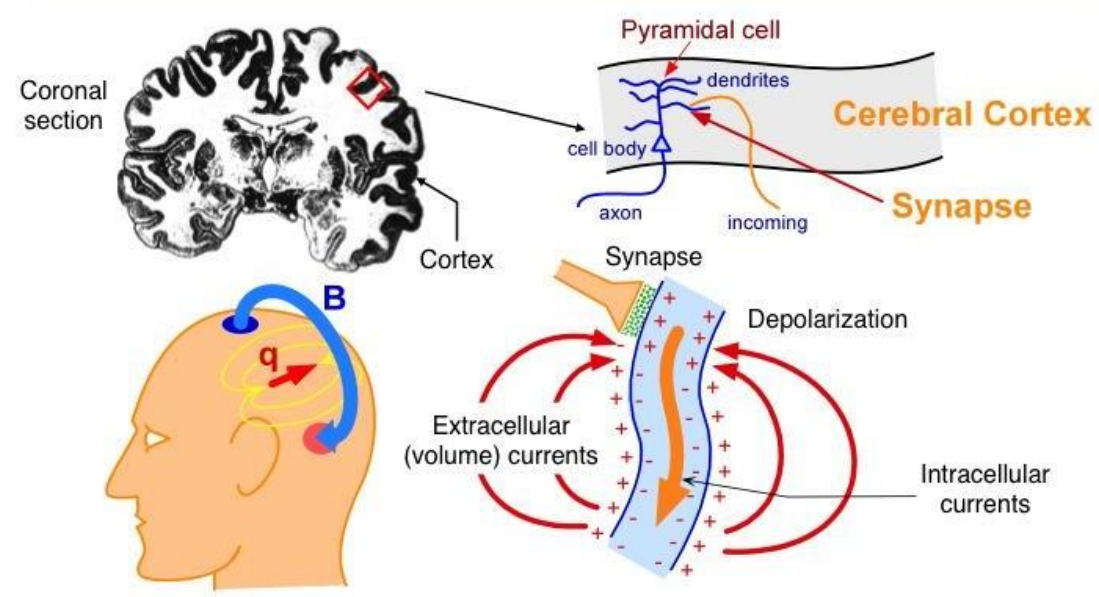

Figure 4. Origin of MEG Signals.

Source: http://ilabs.washington.edu/what-magnetoencephalography-meg 


\section{How to measure brain activity with MEG}

Once we have a cortical patch of enough neurons firing at about the same time and generating currents that have a similar orientation, measurable electrical currents will sum up, producing a field large enough to be detected outside the head with MEG. We can measure the magnetic field generated from these currents if we take into account Maxwell's right-hand rule that a magnetic fields curves around the axis of the electrical currents ${ }^{79}$. However, the magnetic fields so generated by the brain are extremely tiny, typically about 50-500 fT, which is about 100 million times weaker than the earth's magnetic field. Thus, its detection requires high-end instrumentation that can provide high sensitivity and the ability to suppress interference from the outside several orders of magnitude stronger than the signals of interest. Therefore, it is necessary to amplify these small magnetic fields using flux transformers.

A flux transformer consists of a large coil of about $1 \mathrm{~cm}^{2}$ that is wired to a considerably smaller coil. These coils are both made of superconductive metal that it is cooled down to almost absolute zero (about 4 Kelvin), temperatures at which these materials become superconducting ${ }^{80}$. The bigger coil is positioned as close as possible to the head where it can best pick up the weak magnetic fields of the brain. Because of the superconductivity, there is absolutely no resistance in the system in the two coils and consequently, no total magnetic fields can be produced within the system. Therefore, any magnetic field that enters the big coil will result in the same magnetic field with opposite direction in the small coil and because of the smaller size of the second coil, the flux will be stronger and amplified (Figure 5) ${ }^{81}$. It is this amplified field that can be picked up using Superconducting Quantum Interference Devices (SQUIDS). 


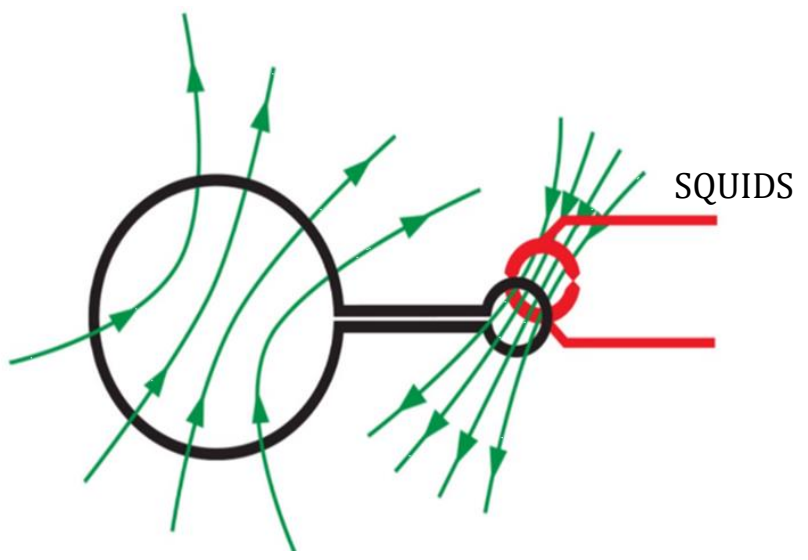

Figure 5. Flux Transformer

Thus, SQUIDS, the ultrasensitive detectors of magnetic fields, are connected to the superconducting coils with smart sensor designs and they are able to pick up the magnetic signals generated by the cortical currents. The current MEG systems contain more than 300 SQUIDs in helmet-shaped arrays, so that signals can be recorded simultaneously over the whole cortex, often sampled at up to 30 $\mathrm{kHz}$ simultaneously.

SQUIDs implement the principles of quantum physics for the detection of small electrical currents, like those induced by weak magnetic signals, with high sensitivity and large dynamic ranges. To better understand how SQUIDS work, it is important to highlight some characteristics of the superconductive materials. When these materials are cooled down to a sufficiently low temperature, they lose electrical resistance completely; becoming superconductors ${ }^{78,80}$. An electric current will flow in these materials without friction and when arranged to circulate in a loop, the current does so infinitely, if the low temperature is maintained. This never-ending current, like any other electric current, generates a magnetic field. The opposite is also true with superconductors materials: an applied static magnetic field will give rise to a continuous shielding current on the surface of a superconductor, which in turn will generate a magnetic field exactly canceling the external field, preventing the external field from entering the superconducting material 78 . 
The critical temperature $T \mathrm{c}$, at which the material changes from its normal to the superconducting state, depends on the material. Normally, the material used as superconductor in the SQUIDs, in MEG as well as MRI, is the niobium ${ }^{82}$. This element has a critical temperature of $8 \mathrm{~K}$ and it allows greater penetration of the magnetic field. Also, the cooling element most commonly used is liquid helium, whose boiling point is $4.2 \mathrm{~K}$ or $-269^{\circ} \mathrm{C}$. The transition between the normal and superconducting states is not only regulated by the temperature. Superconductors also have a characteristic critical field $B c$ (typically ranging between $1 \mathrm{mT}$ and 100 T) and a critical current Ic; if these limits are exceeded, the material will return to its normal state, even if the temperature is preserved below the critical value. Thus, superconductors will not support arbitrarily large magnetic fields passing through them ${ }^{78-80,83}$.

To optimize the sensitivity, SQUIDs are made rather small, typically less than $1 \mathrm{~mm}$ in outer diameter. Due to the small surface area, SQUIDs present a poor coupling to the magnetic field. In MEG applications, SQUIDs cannot be used as they are; as mentioned earlier, the coupling must be enhanced with flux transformers that squeeze more magnetic flux into the SQUID loop by collecting it from a much larger area (Figure 5).

Thus, the simplest sensor configuration of a flux transformer is called magnetometer, which senses the magnetic field with a single coil loop, thus measuring one component of the magnetic field in one location. A magnetometer is also sensitive to homogeneous fields, which are often caused by distant noise sources 80,81 .
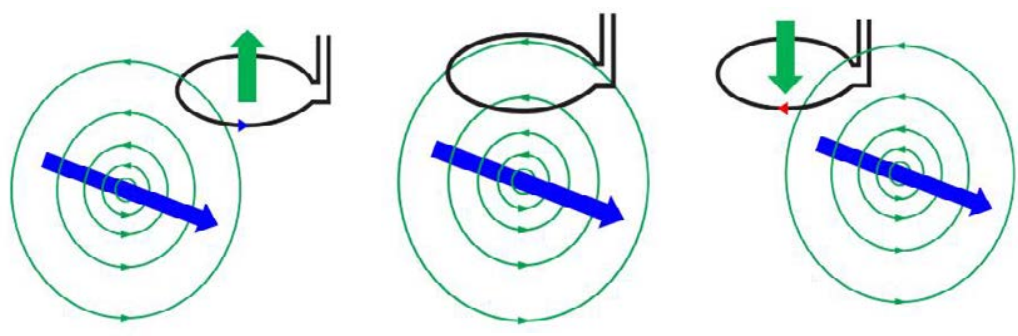

Figure 6. Magnetometer 
In figure 6 we see that if we have an active group of neurons generating electrical currents (green circles) which in turn create a magnetic field (blue arrow), should the magnetometer (the big coil of the flux transformer) be placed rather off center to the source, the magnetic field will pass through it in an upward direction (Figure 6a). These upward magnetic fields can be picked up by the SQUID that is coupled to the flux transformer. However, if the magnetometer moves straight above the source, the coil will be oriented in the same direction as the magnetic fields and no magnetic flux can be picked up (Figure 6b). Lastly, if the magnetometer moves further to the left, it will pick up the magnetic field that is oriented downwards. Therefore, we can see that the sensitivity of the magnetometer depends on its location relative to the source.

Another configuration is the axial gradiometer, which consists of two coils placed on top of each other along the axial direction with respect to the source, the distance between them being typically about $5 \mathrm{~cm}$. Importantly, they are wired in opposite directions, so that the magnetic fields in the two coils are subtracted from each other, making the gradiometer sensitive to the difference in magnetic field strength $^{78}$. In Figure $7 \mathrm{a}$, the bottom coil will be the most sensitive to the brain since it is the one closest to it. Additionally, as can be seen in Figure 7b, both coils will also pick up ambient noise (purple lines). In the magnetometer configuration, this noise would just have been added onto the measured brain signals but with gradiometers, since both coils pick up this noise equally, it is removed in the measurement of the difference between the coils. This made the gradiometer configuration a very efficient ambient noise suppressor.
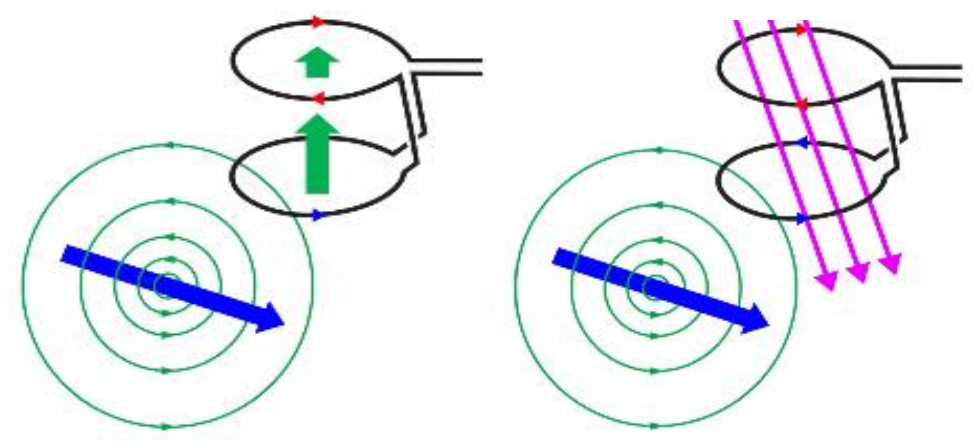

Figure 7. Axial Gradiometer 
Another configuration with similar ambient noise reduction properties is the planar gradiometer. The coils are also wired in opposition to each other and oriented in the same plane. They cancel the effect of the noise in a similar way as the axial gradiometer does, but they have very different sensitivity profile when it comes to the distance to the source ${ }^{79,80}$. Figure 8 a shows that when the planar gradiometer is positioned to the side of the source, it will be able to pick up some of the magnetic field while suppressing activity further away from the source. However, they are most sensitive when they are positioned right above the source. In Figure 8b, the right coil will pick up the outwards oriented magnetic field while the downward oriented field will be picked by the left coil. Thus, the difference between these coils would be additive and the total sensitivity much larger than when it was located on the side of the source.
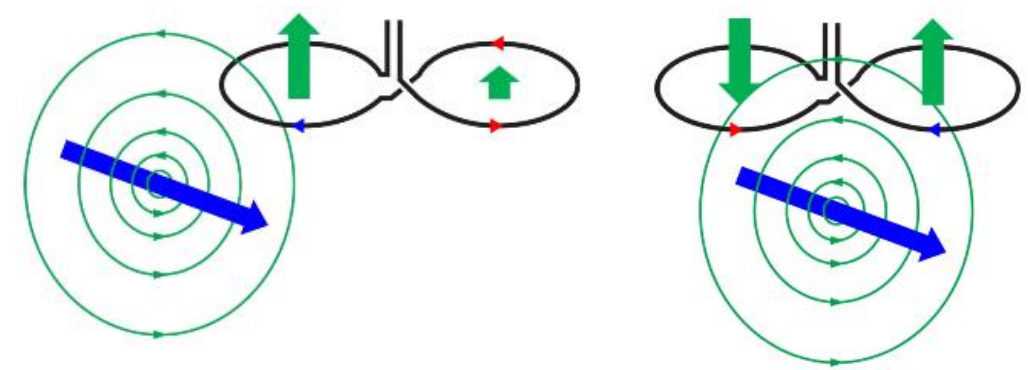

Figure 8. Axial and Planar gradiometers.

By looking at the sensitivity profiles of the different sensors configurations with respect to the distance to the source, the magnetometer will be most sensitive to the source when it is positioned right off its center after which the sensitivity quickly declines with distance, however, it remains sensitive to distant magnetic fields. They could originate in principle from brain sources but probably also contain a lot of noise. On the other hand, the axial gradiometer suppresses effectively the distant noise because its sensitivity to magnetic fields declines more rapidly with distance. Finally, the planar gradiometer is another option for suppressing distant noise and it is most sensitive when it is located right above the source. Because of the ambient noise suppression, most MEG systems employ 
these gradiometers rather than the magnetometers. However, some of the most common MEG systems nowadays combine sensors to obtain a better measurement of neural activity and ambient noise suppression.

To maintain the low temperature of the instrumentation, a special container called Dewar is needed. It comprises two concentric vessels with a vacuum jacket and radiation shields in between. The vacuum prevents heat conduction from the outside to the inside vessel, and the shields block thermal radiation. The sensors reside in the inner vessel 2 immersed in the liquid helium $(\mathrm{T}=4.2 \mathrm{~K})$. A MEG Dewar must be strictly nonmagnetic to avoid distorting the fields being measured. The Dewar is usually built of glass-fiber composites which is magnetically transparent and has a capacity of 70-100 L of liquid helium ${ }^{78}$.

The mechanical system supporting the Dewar is called the gantry. It often allows adjusting the elevation and angle of the Dewar to accommodate subjects of different heights and in different measurement positions, such as seated or supine. Alternatively, the height adjustment can be addressed by moving the seat up/down. The gantry should be very rigid, since any movements of the sensors in the remnant field inside the shielded room gives rise to artifacts ${ }^{78}$.

Due to all the ambient noise that can affect the MEG system and the measurement of the small magnetic fields produced in the brain, it is necessary to isolate the MEG in a magnetically shielded room (MSR).

The MSR improves the strength of the useful signal by excluding the background noise and other spurious magnetic fields. An MSR is an enclosure with a shell comprising layers of high permeability metals that are also good electrical conductors. This attenuates the spurious magnetic and electrical fields emanating from numerous external sources in buildings such as hospitals.

Among all the MEG systems, Elekta Neuromag is one of the most used MEG. The last two studies of this thesis are performed with this system.

Elekta Neuromag MEG (Figure 9) has 306-channel contained in a magnetically shielded room. The system optimally combines the focal sensitivity of 204 planar gradiometers and the widespread sensitivity of 102 magnetometers in a helmet-shaped array covering the entire scalp. A 3D-digitizer (FASTRACK; 
Polhemus) and four HPI coils enable the continuous monitoring of the head position inside the MEG. The system is integrated with simultaneous high-density EEG (up to 128 channels), and four differential channels, so that EEG, EOG, EMG, and ECG signals can be recorded. The participant's behavior inside the MEG as well as their online responses can be video-recorded by using two independent opticalfiber-based digit-response pads.
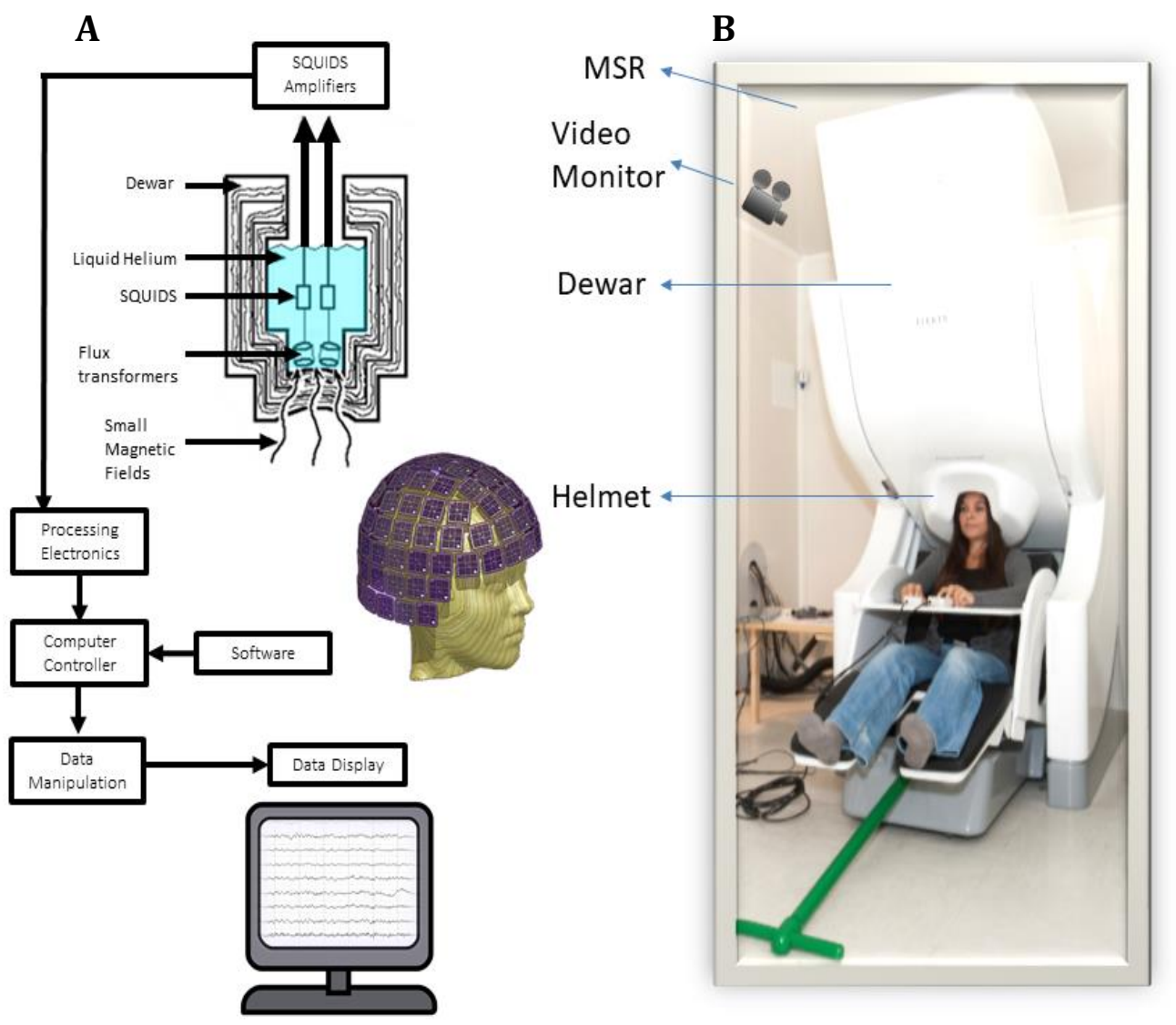

Figure 9. MEG System. A. The largest bulk of the systems consists of the Dewar containing the liquid helium for cooling down the flux transformers in the squids. From the squids on, the measured signal is amplified and then digitized and passed on to computers for display, storage, and analysis. B. MSR. The MEG system is placed in a magnetically shielded room that protect the MEG from all devices that might generate the magnetic fields placed outside it, to reduce interference with the measurements. 


\section{Brain Rhythms}

The fluctuations of the neuronal activity generated when the neurons are firing in a synchronized way, give rise to the brain rhythms registered with MEG and EEG.

Hans Berger in 1929, observed brain waves over the occipital cortex in his young son. He was the first to observe alpha rhythm, regular sine waves that oscillate between 8 and $12 \mathrm{~Hz}$, when his son closed his eyes. When he opened his eyes, the rhythm disappears, showing only complex and fast activity ${ }^{84}$.

The brain produces oscillatory activity in two ways, by intrinsic mechanisms within individual neurons or interactions between neurons. First, inherent mechanisms of individual neurons include oscillations in membrane potentials or action potentials which later generate oscillations of the postsynaptic neurons. The interaction between neurons can develop macroscopic oscillations, the ones we can see with MEG.

In general, neural oscillations can be characterized by their frequency, amplitude, and phase. These signal properties can be extracted from neural recordings using time-frequency analysis and these characteristics will be explored later in this thesis, especially the phase.

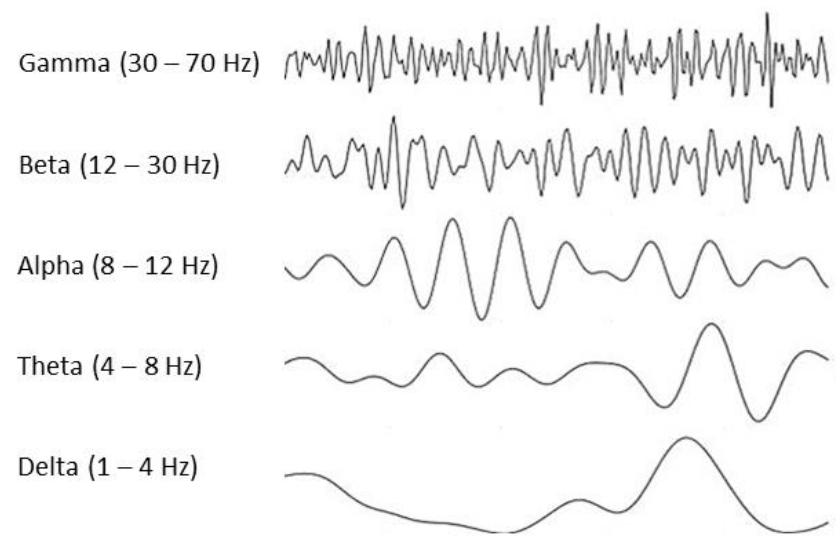

Figure 10. Brain rhythms and classical definition of frequency bands

MEG signals have a wide spectral content, but also reveal oscillatory activity in specific frequency bands: 
- Delta $(1-4 \mathrm{~Hz})$ : This is the slowest and highest amplitude classically described brain rhythm, usually associated with the deep stages of sleep. In addition, delta waves are involved in regulating unconscious bodily processes such as heartbeat, kidney, and digestive functioning. Infants tend to have more delta activity and as people get older, delta rhythm becomes sparser even during sleep. Apart from these basic functions, delta rhythm has been linked to cognitive processes: for instance, several studies have found increased delta power in mental calculation, semantic and attentional tasks ${ }^{85-87}$.

- Theta $(4-8 \mathrm{~Hz})$ : two types of theta rhythms have been described. The hippocampal theta rhythm is a strong oscillation that can be found in the hippocampus, and the cortical theta rhythm can be found as slow frequency activity recorded in the scalp. Theta activity has been associated with cognitive processing during memory tasks ${ }^{88-91}$.

- Alpha (8 - 12 Hz): The alpha rhythm, whose name is because it was the first observed, is the dominant one during resting state and in some meditative states. Alpha is involved in mental coordination, learning and in the perception of visual stimuli. Thus, alpha power is strongly modulated by eye-opening, which triggers a decrease in alpha rhythm. Moreover, attention to visual stimuli causes changes in alpha activity ${ }^{92}$.

- Beta $(12-30 \mathrm{~Hz})$ : The beta band comprises the high frequency and low amplitude brain rhythms that are commonly observed while we are awake. They are involved in conscious thought and logical thinking. Most people exhibit the beta rhythm throughout the day to complete conscious tasks such as critical thinking, writing, reading, and socialization ${ }^{93,94}$.

- Gamma (30 - $100 \mathrm{~Hz}$ ): This brain rhythm is involved in higher processing tasks as well as cognitive functioning. Gamma is important for learning, memory and information processing ${ }^{95}$. 


\section{MEG signal pre-processing}

Once the MEG data have been recorded, the next step consists in cleaning the signal. Sometimes, during a recording, the signal obtained may be contaminated with artifacts due to the movements of the subjects, blinks, heartbeats, powerline and many others possible disturbances.

External disturbances in the proximity of the MEG sensors can cause strong and spatially complex artifacts that are difficult to remove with the existing interference-suppression methods. The first method in our preprocessing pipeline is the signal space separation provided in MaxFilter by the Elekta Software ${ }^{96}$.

\section{Signal Space Separation (SSS)}

Maxfilter ${ }^{\circledR}$ is an Elekta Neuromag proprietary filter designed to suppress magnetic interference and artifacts coming from inside and outside of the MEG sensors, to transform data between different head positions, and to compensate disturbances due to magnetized material on the head and due to head movements ${ }^{96,97}$.

First, MEG sensor data is visually inspected to find broken and noisy channels to be subsequently excluded from the filtering. Nevertheless, the components obtained by the signal space separation process (SSS) ${ }^{91}$ are projected to all MEG sensors, and all 306 sensors can be used in the subsequent analysis.

Briefly, SSS is based on the fundamental properties of electromagnetic fields and harmonic function expansions ${ }^{98}$ to separate the MEG data into three elements (Figure 11): brain magnetic fields originating inside the sensor array (bin), external artifacts produced outside it (bout) and the noise generated by the sensors and sources of interference located very close to the sensors (n). Disturbances are removed by omitting the harmonic function components corresponding to excessively high spatial frequencies, by rejecting the $\boldsymbol{S}_{\text {out }}$-space component $\mathbf{b}_{\text {out }}$ and by reducing the $\boldsymbol{S}_{\mathbf{T}}$ space component (Figure 11 ) ${ }^{96,98}$. 


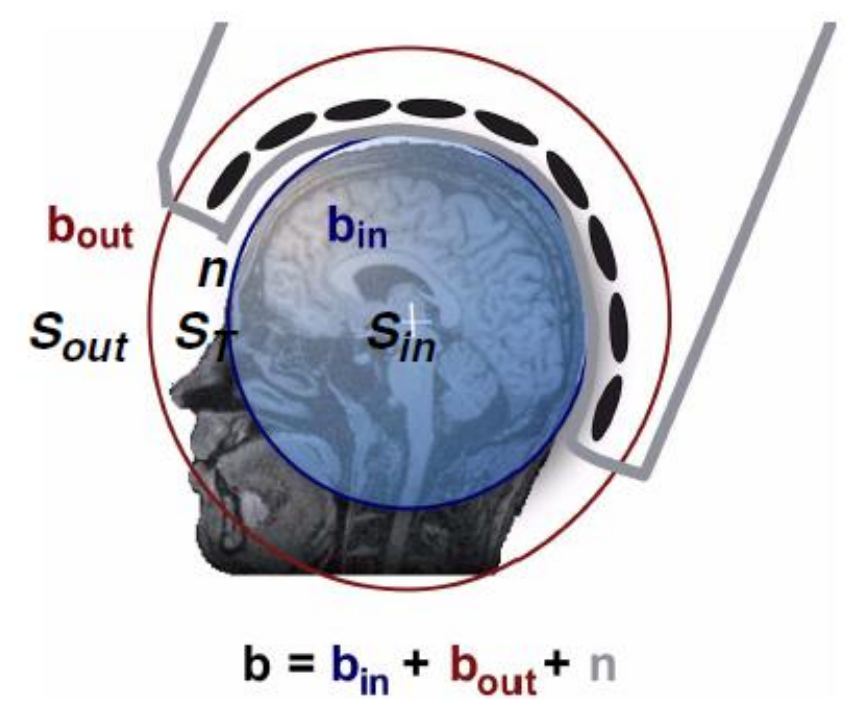

Figure 11. Maxfilter Source: Elekta-Neuromag Manual

Maxfilter can be considered as a spatial filtering because the separation of $\mathbf{b}_{\text {in }}$ and $\mathbf{b}_{\text {out }}$ is carried out based on the spatial patterns and is time-independent. Spatial separation can suppress only external interference emanating from space Sout, such as electromagnetic pollution due to power lines, radio communication, traffic, elevators etc. External disturbances can also originate in the patient, as heartbeats and muscular activity cause fields detectable by MEG sensors, and any pieces of magnetized material that can be in the body of the subject and may cause very large disturbances ${ }^{98,99}$.

To identify and suppress $\mathbf{S}_{\text {out }}$ components it is important to have additional knowledge about their temporal dynamics. The temporal extension of the SSS method (tSSS) removes also artifacts that generate in the $\boldsymbol{S}_{\boldsymbol{T}}$ space or very close to it. Such disturbances can be caused, for example, by magnetized pieces on the subject's head like dental work, pacemakers or stimulators attached to the patient ${ }^{100}$. Finally, tSSS allows for movement compensation, by continuously tracking the position of the head relative to the MEG sensor array and projecting the signals into the original sensor positions. tSSS is the method applied to last two studies presented in this thesis using the default Maxfilter parameters. 


\section{Artifact detection and cleaning}

The next step after removing the external disturbances with Maxfilter software is to eliminate artifacts coming inherently from the subjects like blinks, heartbeats and muscular activity. This can be done by visual inspection where a MEG expert explores the whole recording looking for any of these artifacts and with automatic artifact rejection methods.

Thus, if an event is repetitive and occurs always in the same place, such as eye blinking and heartbeats, it is possible to identify the topographies corresponding to these artifacts and remove them from the recordings. The spatial decomposition is the basic idea behind two broadly used methods: The Signal Space Projection (SSP) and the Independent Component Analysis (ICA), which were used in this thesis. We describe them below.

\section{Signal Space Projection}

SSP works with the idea that the spatial distributions of the magnetic field emerging from the brain sources are sufficiently different from the ones obtained from external noise sources. Moreover, it implicitly assumes that the linear space spanned by the significant external noise patterns has a low dimension ${ }^{101 .}$

The foundation of this method is now briefly described ${ }^{102}$ : let's suppose we can always decompose any n-channel measurement $b(t)$ into its signal and noise components as:

$$
b(t)=b_{s}(t)+b_{n}(t)
$$

Knowing that $b_{n}(t)$ is well characterized by a few field patterns $\mathrm{b}_{1}, \ldots, \mathrm{b}_{\mathrm{m}}$, the noise can be expressed as:

$$
b_{n}(t)=U C_{n}(t)+e(t)
$$


where the columns of $U$ constitute an orthonormal basis for $\mathrm{b}_{1}, \ldots, \mathrm{b}_{\mathrm{m}}, C_{n}(t)$ is an $\mathrm{m}$ component column vector, and the error term $e(t)$ is small and does not exhibit any consistent spatial distribution over time ${ }^{102}$.

Then, we call the column space of $U$ the noise subspace. The basic idea of SSP is that we can find a small basis set $b_{1}, \ldots, b_{m}$ such that the conditions described above are satisfied. We can now construct the orthogonal complement operator ${ }^{102}$ :

$$
P \perp=I-U U^{T}
$$

and apply it to $b(t)$ yielding:

$$
b(t) \approx P \perp b_{S}(t)
$$

since $P \perp b_{n}(t)=P \perp U C_{n}(t) \approx 0$. The projection operator $P \perp$ is called the SSP projection operator and generally provides considerable rejection of noise, suppressing external disturbances by a factor of 10 or more. The effectiveness of SSP depends on two factors ${ }^{102}$ :

- The basis set $b_{1}, \ldots, b_{m}$ should be able to characterize the artifacts field patterns completely.

- The angles between the noise subspace space spanned by $b_{1}, \ldots, b_{m}$ and the signal vectors $b_{s}(t)$ should be as close to $\pi / 2$ as possible.

If the first condition is not satisfied, some noise will leak through, because $P \perp$ $b_{n}(t) \neq 0$. If any of the brain signal vectors $b_{s}(t)$ is close to the noise subspace not only the noise but also the signal will be attenuated by the application of $P \perp$ and, consequently, there might by little gain in signal-to-noise ratio ${ }^{101,102}$. 


\section{Independent Component Analysis}

Another commonly used preprocessing technique consists in carrying out a linear decomposition of the MEG data using independent component analysis (ICA). In this section, ICA will be briefly described.

ICA relies on the same logic as the SSP method mentioned earlier: identifying spatial topographies that are specific from an artifact and then removing them from the MEG data. ICA tries to identify components independent in time.

Thus, ICA components are linear combinations of several channels acting as spatial filters on the MEG data. ICA puts different weights on each channel making each component independent from all other components.

In this case, these spatial filters are chosen to produce the maximally temporally independent signals available from the MEG sensor data. Certain artifacts, such as those caused by eye blinking, are often reflected by a few components, which are, with some experience, easy to identify. These visually identified components can then be removed from the data and the remaining components can be projected back to the sensor level, as described henceforth ${ }^{103,104}$.

Let sensor data be noted as $X_{k}(i)$ denote a MEG sensor $(i=1, . ., L$ is the number of sensors used, and $k$ denotes discrete time). Each $X_{k}(i)$ is expressed as the weighted sum of $M$ independent signals $S_{k}(j)$, following the vector expression:

$$
X_{k}=\sum_{j=1}^{M} a(j) S_{k}(j)=A S_{k}
$$

where $X_{k}=\left[X_{k}(1), \ldots, X_{k}(L)\right]^{T}$ is an L-dimensional data vector, from the $L$ mixtures at time $k$. The $S_{k}(1), \ldots, S_{k}(M)$ are the $M$ zero mean independent source signals, and $A=[a(1), \ldots, a(M)]$ is a mixing matrix independent of time whose elements $a_{i j}$ are the unknown coefficients of the mixtures. To perform ICA, it is necessary to have at least as many mixtures as there are independent sources $(L \sim$ 
M). When this relation is not fully guaranteed, and the dimensionality of the problem is high enough, we should expect the first independent components to present clearly the most strongly independent signals, while the last components still consist of mixtures of the remaining signals ${ }^{104,105}$.

The mixing matrix $\mathrm{A}$ is a function of the geometry of the sources and the electrical conductivities of the brain, cerebrospinal fluid, skull, and scalp. Although this matrix is unknown, here we assume it to be constant or slowly changing (to preserve some local constancy) ${ }^{104}$. Then, after estimating the matrix A, we can calculate its inverse $\mathrm{B}$, and get the independent component simply by:

$$
\hat{S}_{k}=B X_{k}
$$

The statistical model in (6) is what we call ICA model. The ICA model is a generative model, meaning it describes how the observed data are generated by a process of mixing the components in $S_{k}$.

\section{Source Reconstruction}

An advantage of MEG is that allows the localization of active brain regions with feasible spatial accuracy, together with the extraction of the source time series of those areas with exceptional temporal accuracy. Thus, source reconstruction is an important step in the analysis of MEG data. Certainly, the decomposition of the MEG sensor data into specific anatomic brain regions activation allows to clarify the origin in the brain of the stimulus and also the task effects. It is important to keep in mind that with MEG data is possible to estimate the center of an active brain area but we cannot know specific information about its shape. The resultant activity map is defined then by the specific source reconstruction method employed ${ }^{78}$.

\section{Forward Model}

The first step to reconstruct the MEG neural sources is to obtain a model that explain how the neuronal currents produce magnetic fields read by the MEG 
sensors, given the different head tissues including white and grey matter, CSF, skull bone and skin. This is called the forward model (Figure 12) which explains, how MEG data values can be obtained outside of the head from electrical current dipoles in the brain ${ }^{106}$. In this context, MEG and EEG physics can be explained through the quasi-static approximation of Maxwell's equations. The quasi-static current flow $J\left(r^{\prime}\right)$ at location $r^{\prime}$ is therefore divergence-free and can be related to the magnetic field $B(r)$ at location $r$ through the well-known Biot-Savart law ${ }^{107}$ :

$$
B(r)=\frac{\mu_{0}}{4 \pi} \int J\left(r^{\prime}\right) \times \frac{r-r^{\prime}}{\left\|r-r^{\prime}\right\|^{3}} d v^{\prime}
$$

where $\mu_{0}$ is the permittivity of free space. We can partition the total current density in the head volume into two current flows of distinct physiological significance: a primary current flow $J^{P}\left(r^{\prime}\right)$ related to the original neural activity and a volume current flow $J^{V}\left(r^{\prime}\right)$ that yields from the effect of the electric field in the volume on the action potential ${ }^{107}$ :

$$
J\left(r^{\prime}\right)=J^{P}\left(r^{\prime}\right)+J^{V}\left(r^{\prime}\right)=J^{P}\left(r^{\prime}\right)+\sigma\left(r^{\prime}\right) E\left(r^{\prime}\right)=J^{P}\left(r^{\prime}\right)-\sigma\left(r^{\prime}\right) \nabla V\left(r^{\prime}\right)
$$

where $\sigma\left(r^{\prime}\right)$ is the conductivity profile of the head tissues, which we will assume, for simplicity, to be isotropic, and the electric field $E\left(r^{\prime}\right)$ is the negative gradient of the electric potential, $V\left(r^{\prime}\right)$.

If we assume that the head consists of a set of contiguous regions each of them with a constant isotropic conductivity $\sigma_{i}=1, \ldots, 3$, representing the brain, skull and scalp, we can rewrite the Biot-Savart law above as a sum of contributions from the primary and volume currents ${ }^{79}$ :

$$
B(r)=B_{0}(r)+\frac{\mu_{0}}{4 \pi} \sum_{i j}\left(\sigma_{i}-\sigma_{j}\right) \int_{S_{i j}} V\left(r^{\prime}\right) \frac{r-r^{\prime}}{\left\|r-r^{\prime}\right\|^{3}} \times d S^{\prime}{ }_{i j}
$$


where $B_{0}(r)$ is the magnetic field due to the primary current. The second term is the volume current contribution to the magnetic field formed as a sum of surface integrals over the brain-skull, skull-scalp, and scalp-air boundaries.

This equation states that the magnetic field can be calculated if we know the primary current distribution and the potential $V\left(r^{\prime}\right)$ on all surfaces. We can create a similar equation for the potential only ${ }^{79}$, yielding:

$$
\left(\sigma_{i}-\sigma_{j}\right) V(r)=2 \sigma_{0} V_{0}(r)-\frac{1}{2 \pi} \sum_{i j}\left(\sigma_{i}-\sigma_{j}\right) \int_{S_{i j}} V\left(r^{\prime}\right) \frac{r-r^{\prime}}{\left\|r-r^{\prime}\right\|^{3}} \times d S_{i j}^{\prime}
$$

for the potential on surface $S_{i j}$ where $V_{0}(r)$ is the potential at $\mathrm{r}$ due to the primary current distribution.

Thus, Eq. (9) and (10) will represent the integral solutions to the forward problem. When a primary current distribution $J^{P}\left(r^{\prime}\right)$ is specified, it is possible to calculate a primary potential and a primary magnetic field ${ }^{107}$ :

$$
\begin{gathered}
V_{0}(r)=\frac{1}{4 \pi \sigma_{0}} \int J^{P}\left(r^{\prime}\right) \cdot \frac{r-r^{\prime}}{\left\|r-r^{\prime}\right\|^{3}} d r^{\prime} \\
B_{0}(r)=\frac{\mu_{0}}{4 \pi} \int J^{P}\left(r^{\prime}\right) \times \frac{r-r^{\prime}}{\left\|r-r^{\prime}\right\|^{3}} d r^{\prime}
\end{gathered}
$$

the primary potential $V_{0}(r)$ is then used to solve Eq. (10) for the potentials on all surfaces. These surface potentials $V(r)$ and the primary magnetic field $B_{0}(r)$ are then used to solve Eq. (9) for the external magnetic fields.

\section{Spherical Head Models}

Nowadays, there are several methods to obtain a forward model. Some of them employ 3D brain volumes while others constrain the sources to a 2D space of the cortical surface using MRI ${ }^{108}$. In addition, there are other approaches that involves head models which use Maxwell's equations ${ }^{79}$ to explain how the magnetic fields are produced by the sources at each MEG sensor as mentioned above. Some of these methods are based on analytical solutions for spherical 
surfaces, or on numerical solutions for a Boundary Element Model (BEM) approximation to the head ${ }^{106}$. There are further numerical methods such as Finite Element Model ${ }^{109}$, but they will not be used in this thesis and therefore we will not describe them here.
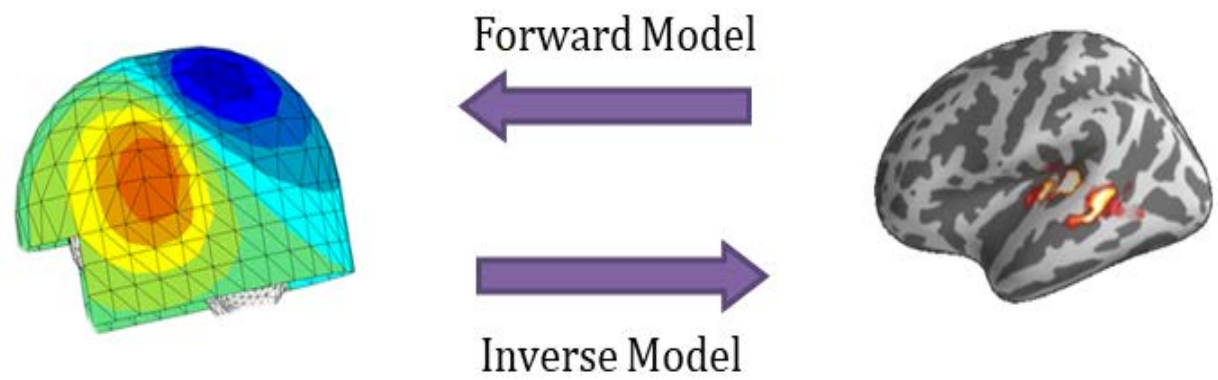

Figure 12. Forward and Inverse Model

Because of its simplicity in calculations, we have resorted to models using spheres to solve the forward problem in MEG, although the brain is not a sphere but is a good approximation. It can be noticed that most brains can fit reasonably inside a sphere using the fiducial points (nasion, and both bilateral pre-auricular points) obtained at the beginning of the MEG recording. The spherical head models work reasonably well, particularly for MEG recordings, which are less sensitive than EEG to the effects of volume currents. In MEG, the radial component of the magnetic field is not affected by the volume currents, and therefore it will only depend on the primary current source. These spherical methods have the advantage that the computation of the forward problem can be simplified ${ }^{110}$.

Let us consider the special case of a current dipole of moment $q$ located at $r_{q}$ in a multi-shell spherical head, and a MEG system in which we measure only the radial component of the magnetic field (Figure 13). It is relatively straightforward to show that the contributions of the volume currents (from Eq. (9)) vanish in this case, and we are left with only the primary term, $B_{0}(r)$. Taking the radial component of this field for the current dipole reduces to the simple analytical formula107:

$$
B_{r}(r) \equiv \frac{r}{r} \cdot B(r)=\frac{r}{r} \cdot B_{0}(r)=\frac{\mu_{0}}{4 \pi} \frac{r \times r_{q}}{r\left\|r-r_{q}\right\|^{3}} \cdot q
$$


From the equation above we can observed that the magnetic field measured is linear in the dipole moment $q$ but nonlinear with respect to its location $r_{q}{ }^{107}$.

From Eq. 13 we can also see that, due to the triple scalar product, $B_{r}(r)$ is zero everywhere outside the head if $q$ points towards the radial direction $r_{q}$. In other words, radially oriented dipoles do not produce any external magnetic field outside a spherically symmetric volume conductor, regardless of the sensor orientation ${ }^{111}$. Therefore, signals from currents at the crests of the gyri and depth of the sulci are attenuated in the MEG data ${ }^{78,106 .}$

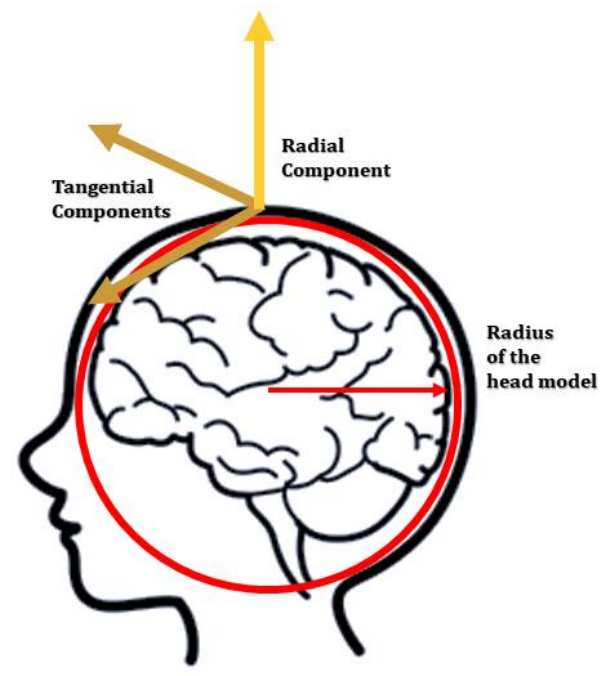

Figure 13. Radial component of the magnetic field in MEG

Additionally to the spherical head models, the overlapping sphere method provides another solution to the numerical approaches. The overlapping spheres method is based on the estimation of a different sphere for each MEG sensor. It is possible to imagine that the best fitting sphere for the occipital region of the brain will be different from the best fitting sphere for the frontal region. Thus, instead of using only one sphere for the whole head, this method estimates a sphere that fits locally the shape of the head in the surroundings of each sensor. The forward model for an arbitrary dipole point is then solved using the simpler sphere solution assigned to that sensor ${ }^{112}$. We used this method in the two first studies of this thesis. 
On the other hand, BEM is a method that improves the localization accuracy of the source reconstruction results by introducing a more realistic head volumeconductor model compared to the spherical model. The volume-conductor properties are approximated by realistically shaped compartments of isotropic and homogeneous conductivities. Therefore, the method is able to model at least the shape features of the volume conductor, while neglecting the anisotropy and fine structure of the real tissue, that surrounds the electric sources to be reconstructed 113 .

BEM is often used with EEG due to the effects of the tissues conductivities, which comprises the EEG recordings. Methods using spheres have shown great performance, low computational costs and efficient and reasonable results when solving the forward problem in MEG data.

\section{Inverse Model}

Once the head model is chosen and computed, the next step consists in explaining how to estimate the brain sources when we only have the information of the MEG sensors. This problem is commonly known as the inverse model (Figure 12).

The idea is to estimate the activity of thousands of dipoles described by the forward model. However, we only have the information of the few hundred MEG sensors as inputs. This problem is ill posed, because, there exist current distributions that do not produce any magnetic field. Therefore, there is no unique solution to the problem of reconstructing where exactly the sources of the MEG signals are localized within the brain. Non-uniqueness is handled by making assumptions about the nature of the sources (number of sources, anatomical and neurophysiological constraints, prior probability density functions, norms, smoothness, correlation, covariance models, sparsity, diversity measures, spatial extent constraints, etc.). Introducing these assumptions on the possible solutions can overcome the non-uniqueness of the inverse problem, excluding this way all solutions except the one that best describe and fits the data. Thus, the brain source reconstruction of MEG signals will depend to some degree on the models chosen 
and on the corresponding assumptions, and therefore will have some degree of uncertainty.

In this way, many solutions to the inverse problem have been proposed in the literature 106,107 , based on different assumptions on the way the brain works and depending on the amount of information we already have on the effects we are studying. Among the many methods available, we present in this thesis two general approaches to the inverse problem that represent the most widely used methods in MEG/EEG source imaging: minimum-norm and beamformers.

These approaches have the advantage of being implemented in an efficient linear form: the activity of the sources is a linear recombination of the MEG recordings, such that it is possible to solve the inverse problem by applying a linear kernel (in the form of a matrix that multiples the spatial data at each point in time).

In this way, to estimate the primary current distribution described by $j$ the following linear equation must be solved:

$$
M=L j
$$

where $M$ is the MEG sensors data vector, $L$ is the result from the forward model mentioned in the previous section (often called the leadfield matrix), and $j$ the source current density vector ${ }^{79,111}$ which is an approximation of a naturally continuous current distribution. In some models, $j$ typically contains by far more elements than there are sensors. Consequently, there are distributions $j_{0} \neq 0$ that do not produce any measurable signals, for which $L j_{0}=0$, which constitutes the "non-uniqueness" of the underdetermined inverse problem ${ }^{114,115}$. Therefore, the idea is to find an estimate $\hat{\jmath}$ that comes as close as possible to the real but unknown current distribution $j$, given the MEG data and possibly further a priori assumptions $^{116 .}$

Thus, linear estimation methods attempt to find such an estimate by multiplying the data by an inverse operator matrix $G^{116}$ :

$$
\hat{\jmath}=G M
$$




\section{Minimum Norm Estimation (MNE)}

The MNE is considered a distributed source model designed to solve the inverse problem. The least-squares MNE was first introduced to MEG in the source reconstruction analysis by Hämäläinen and Ilmoniemi117. In general terms, this approach is a distributed inverse solution that discretizes the source space into locations in each point of the source grid (either brain volume or surface) using a large number of equivalent current dipoles. It estimates the amplitude of all modeled source locations simultaneously and recovers a source distribution with minimum overall energy that produces data consistent with the MEG measurement.

This approach finds a cortical current source density image that approximately fits the data when mapped through the forward model $L$. The nonuniqueness here is dealt with by introducing a regularization parameter in the form of a source covariance that favors solutions that are of minimum energy, following the equation:

$$
G=L^{T}\left(L L^{T}+\lambda C\right)^{-1}
$$

where $\lambda$ is the regularization parameter and $\mathrm{C}$ is the noise covariance matrix.

With this method, the source reconstruction is biased towards superficial cortical sources, since deeper sources require stronger activation than superficial ones to generate the same field intensity in MEG sensors. To deal with this bias, depth-weighting factors or noise normalization has been added to the MNE solution 116,118 .

\section{Beamforming}

An alternative approach to perform source reconstruction of the MEG signals measured at the scalp is to use beamforming. Instead of trying to solve a system of equations with several parameters and constraints on the solution, the 
activation time-course at each source location (and in each direction) can be determined independently of all other locations, by means of spatial filters ${ }^{78}$.

A spatial filter comprises a set of weights $\left(w=\left[w_{1}, w_{2}, \ldots, w_{N},\right]^{T}\right)$ that define a linear combination of the brain signals at all MEG sensors; this is supposed to enhance the activity at that specific source while suppressing activity from sources at other locations. Thus, the activation time course $q(t)$ of a source at a given source (grid) location $i$ is estimated as ${ }^{78}$ :

$$
q_{i}(t)=w_{i}^{T} M(t)
$$

where $q(t)$ is the source activity and $M(t)$ are the MEG measurements.

To obtain a distributed representation of source activity using this method, the idea is to scan through the brain volume and build a set of spatial filter weights (beamformers) $w_{j}$ for each point and orientation of the grid. With these weights, Eq.17 is used to compute the source activity.

Linearly constrained minimum variance (LCMV) beamformer is one of the most common and used beamforming methods. This approach computes an estimate of source activity at each sensor location through spatial filtering. The spatial data are linearly combined with weights (the spatial filter) chosen separately for each location to ensure that the strength of a dipolar source at that location is correctly estimated. The remaining degrees of freedom in selecting the weights are used to minimize the total output power. This has the effect of suppressing contributions of sources from other locations to the estimated signal at the location of interest. It should be noted however, that correlation between sources can at times lead to partial or full signal cancellation and the method can be sensitive to accuracy of the head model ${ }^{119}$.

LCMV beamformers require specification of the data covariance matrix, which is assumed to include contributions from background noise and the brain signals of interest. In practice, the data covariance is estimated directly from the recordings. A linear kernel is formed from this data covariance matrix and the 
forward model. This kernel defines the spatial filters applied at each location. Multiplying by the data produces an output beamformer scanning image ${ }^{119}$.

\section{Functional Connectivity (FC)}

In the beginning of the current section, we discussed how MEG signals were generated and obtained and how it was necessary a big population of neurons firing synchronously to generate measurable (albeit small) magnetic fields. Recently, in the study of these neurophysiological signals, researchers have focused on the way neurons communicate with each other. Within this communication, the neural synchronization opens up a new and interesting field of research. In this sense, MEG offers a good perspective due to the high temporal resolution, this technique allows to track rapid changes in the activity of neural populations and to observe changes in the oscillatory activity ${ }^{78}$.

In the study of signal processing, MEG signals at the sensors level have been used to analyze and characterize oscillatory interactions between two signals. Specifically, to characterize brain functional connectivity patterns between brain regions. In this context, $\mathrm{FC}$ is defined as the statistical dependence between two time series providing information about the functional interaction between brain regions.

Unfortunately, there are some limitations when analyzing MEG signals at the sensor level. First, the information obtained from the sensors is just a bidimensional projection, which means we do not know exactly from which brain regions the information is coming or if we are capturing information from more than one brain region. The second problem is called volume conduction. For example, a source located at a point in the cortex will cause both a local intracellular current and a volume current that will spread through the surrounding tissues. And this will cause that brain oscillatory activity will spread throughout the brain; this local source will be observed simultaneously from several sensors. This effect is the greater the more powerful or deeper the source is, and can produce spurious connectivity between nearby sensors ${ }^{120}$. 
For that reason, the study of the FC from a source space perspective can solve or attenuate the limitations mentioned above. The first limitation will disappear due to the source reconstruction of the MEG time series and posterior analyses will be focused on anatomically well delimitated brain regions. Additionally, the effect of volume conduction will be reduced using the right forward and inverse model. Therefore, functional connectivity analysis in the source space can give us accurate information of how and which brain regions interact in a specific time.

The estimation of synchronization from neural data can be addressed from different perspectives and to date various methods exist to assess FC. Some methods are based on the values of the signal's in the time domain (such as the correlation coefficient or those based in the concept of generalized synchronization), others analyze the signal's phase (phase synchronization) or are based on information theory and therefore require the estimation of marginal and joint probabilities distributions (such as mutual information and transfer entropy), among others (see ${ }^{121-123}$ for reviews). In this thesis, we will focus particularly on phase synchronization, which provides a frequency-resolved description of the connectivity across different frequency bands.

\section{Phase synchronization}

During the last decade, phase dynamics has played an important role in understanding the interaction of neural processes ${ }^{124,125}$.

When studying the synchronization of two signals it may be possible that their amplitudes are not related at all; whereas their phases may be. To study these relationships, phase synchronization (PS) methods are used. These approaches require that the phase locking condition is fulfilled for any time $t^{121}$ :

$$
\varphi_{n, m}(t)=n \phi_{x}(t)-m \phi_{y}(t) \leq \text { constant }(18)
$$


where $\phi_{x}(t)$ and $\phi_{y}(t)$ are the phases of the signals. Unfortunately, MEG signals can be noisy and present random phase jumps, therefore, it is necessary to analyze instead the distribution of the cyclic relative phase, confining the phase difference to the interval $[0,2 \pi) 121$ :

$$
\varphi_{n, m}^{\prime}(t)=\varphi_{n, m}(t)(\bmod 2 \pi)
$$

The first step to study phase synchronization is to extract the phases of the signals. To this end, phases can be extracted using the Hilbert or wavelet transform. In this thesis, all phases were extracted using the Hilbert transform. With the Hilbert transformation, the real signal $x(t)$ will be transformed with the help of the analytical signal concept into a complex signal defined as:

$$
\begin{gathered}
x(t)=s(t)+j s_{H T}(t)=A(t) e^{j \phi(t)} \\
s_{H T}(t)=\frac{1}{\pi} P . V . \int_{-\infty}^{\infty} \frac{s(t)}{t-t^{\prime}} d t^{\prime}
\end{gathered}
$$

where $s_{H T}(t)$ is the Hilbert Transform of $s(t)$. P.V. indicates that the integral is computed in terms of the Cauchy principal value, which is used to consider the singularity $t=t^{\prime} . A(t)$ represents the instantaneous amplitude and $\phi(t)$ the instantaneous phase of the signal ${ }^{121}$. It is important to note that the instantaneous phase and amplitude are only meaningful when we are analyzing a narrow-band signal $s(t)$. For this reason, it is necessary, before computing the Hilbert transform, to band-pass filter the signal in the frequency band of interest. Next, to assess the phase locking condition mentioned previously, in this thesis the Phase Locking Value (PLV) was used.

PLV is a well-known PS metric. This method uses the relative phase difference between two signals $s_{1}, s_{2}$ and it is defined at time $\mathrm{t}$ as the average value $^{126}$ :

$$
P L V_{s_{1}, s_{2}}=\left|\left\langle e^{j\left(\varphi^{\prime}{ }_{s_{1}, s_{2}}(t)\right)}\right\rangle\right|
$$


where $<>$ represents time average. PLV ranges between 0 and 1 . If the two signals are synchronized in phase, then the relative phase will be distributed in a small portion of the unit circle, and the PLV value will be high. Conversely, if the two signals do not have phase synchronization, the relative phase will be spread throughout the whole circle and the PLV value will be near zero.

The next chapters will present the three studies developed in this thesis to study the progression from MCI to AD. Each chapter includes its own introduction, materials and methods (in which specific methodology will be explain for each study), results and discussion. 


\section{Chapter 3}

\section{Scopolamine effects on functional brain connectivity: a pharmacological model of Alzheimer's disease ${ }^{1}$}

Scopolamine administration may be considered as a psychopharmacological model of Alzheimer's disease (AD). Here, we studied a group of healthy elderly under scopolamine to test whether it elicits similar changes in brain connectivity as those observed in $\mathrm{AD}$, thereby verifying a possible model of $\mathrm{AD}$ impairment. We did it by testing healthy elderly subjects in two experimental conditions: glycopyrrolate (placebo) and scopolamine administration. We then analyzed MEG data corresponding to both conditions in resting-state with eyes closed. This analysis was performed in source space by combining a nonlinear frequency bandspecific measure of functional connectivity (phase locking value, PLV) with network analysis methods. Under scopolamine, FC between several brain areas was significantly reduced as compared to placebo, in most frequency bands analyzed. Besides, regarding the two complex network indices studied (clustering and shortest path length), clustering significantly decreased in the alpha band while shortest path length significantly increased also in alpha band both after scopolamine administration. Overall our findings indicate that both PLV and graph analysis are suitable tools to measure brain connectivity changes induced by scopolamine, which causes alterations in brain connectivity apparently similar to those reported in AD.

1 The results presented in this chapter have been published in the following paper: S. Pusil \& Bajo, R. et al. Scopolamine effects on functional brain connectivity: a pharmacological model of Alzheimer's disease. Sci Rep 5, 9748 (2015). 


\section{Materials and Methods}

The subjects of the present study are a subset of those from Osipova et al. ${ }^{127}$, who were carefully selected according to the quality of the recordings. They consisted of seven healthy right-handed elderly volunteers (59-80 years and 6 females), recruited from a community cultural center for elderly citizens. Scopolamine hydrobromide $(0.3 \mathrm{mg})$ or glycopyrrolate $(0.2 \mathrm{mg})$, which acted as placebo without penetrating the blood-brain barrier, was given intravenously to each subject in a double blind, cross-over design. The subjects were instructed to move as little as possible and remain awake, while keeping their eyes closed. Magnetic fields were then recorded during this resting state using a 122-channel whole-head MEG device (Neuromag Ltd, Finland) confined in a magnetically shielded room. Raw data were sampled at $400 \mathrm{~Hz}$ and band-pass filtered between 0.03-150 Hz. Time-segments containing artifacts (e.g. amplitudes over 3 pT/cm), were considered extra-cerebral noise. Analyses were performed for the commonly used frequency bands, in order to facilitate comparison with other studies: delta $(0.5-4 \mathrm{~Hz})$, theta $(4-8 \mathrm{~Hz})$, alpha $(8-12 \mathrm{~Hz})$, beta $(13-30 \mathrm{~Hz})$ and gamma $(30-45$ $\mathrm{Hz}$ ).

To reconstruct the neural MEG sources, we selected 88 brain regions derived from a BrainVisa atlas ${ }^{128}$, using Brainstorm software ${ }^{129}$ to define anatomical regions of interest. This number of regions was enough to describe the functional brain network, considering the available 122 MEG channels. Besides, an expert neurologist certified its suitability from the point of view of brain functionality. Because brain MRIs of the subjects were not available, we calculated source activity by using the default anatomy in this toolbox, which consisted of the segmented cortical surface (15000 vertices) of the MNI/Colin27 brain ${ }^{130}$. We chose overlapping sphere method (OS) with Brainstorm default parameter settings to solve the forward problem. This approach in combination with minimum-norm method (MNM) provides the best outcome when the MRIs are not available ${ }^{108}$. Next, a noise covariance matrix was calculated to estimate noise level in the MEG recordings. Then, weighted minimum-norm estimation $\mathrm{WMNE}^{131}$ was chosen to compute source activity. We define the grid of sources (dipoles) by the cortex 
surface template of the default anatomy; each vertex of this surface was considered as a dipole. Finally, we extracted the time series from these cortical ROIs as the average of all dipole's signals within each area of the atlas mentioned above.

The abovementioned PLV was used to measure functional connectivity between all 88 brain regions, in each frequency band. First, data were band-pass filtered using a zero-phase distortion finite impulse response filter with a bandwidth of $2 \mathrm{~Hz}$. Then, we obtain the complex analytic signal from each of the brain regions by using the Hilbert transform from Eq. (20) and (21) (see, e.g., ${ }^{121}$ for details). Subsequently, the PLV between the time series $x(k)$ and $y(k)$ from two brain regions is computed with Eq. (22). Finally, the PLV in each of the frequency bands (delta, theta...) was obtained by averaging the corresponding PLVs in the given frequency range.

Graph theory offers many metrics of complex network organization that can be applied to analysis of brain networks derived from MEG/EEG signals among others. Within the framework of graph theory, brain regions are considered to be the nodes and connection links are extracted from the connectivity matrix (in this case from PLV). This matrix (often called as adjacency matrix ${ }^{132}$ ) contains all information of the network: nodes (brain regions) and links (describing the strength of the connection between brain regions). Finally, with this adjacency matrix, network metrics are computed.

Thus, we described the architecture of the functional brain network of the subjects by means of two indices: the clustering coefficient (C) and the average shortest path length (L). The former one is a measure of graph segregation that indicates how the neighborhoods of a node are interconnected. The latter one is an estimation of network integration, which measures the average of all the shortest distances between pairs of nodes in the network ${ }^{133-135}$. We calculated the mentioned network parameters ( $\mathrm{C}$ and $\mathrm{L}$ ) from the weighted matrix (PLV values) using the "Brain Connectivity Toolbox"133 (http://www. brain-connectivitytoolbox.net). The size of the graph was 88 nodes (corresponding with the 88 source space regions described). Indices are un-normalized.

Statistical differences between experimental conditions (scopolamine vs. placebo), were investigated for both $\mathrm{C}$ and $\mathrm{L}$ by means of a paired $\mathrm{t}$-test at the 
$\mathrm{p}<0.05$ level. In the case of PLV, we first applied a Kruskal-Wallis test and further a non-parametric permutation test with 1000 permutations to each of the significantly different PLVs to correct for multiple comparisons across the full range of functional connectivity pairings. In this latter case, we set the level of significance at $\mathrm{p}<0.005$.

Ethics statement: Methods were carried out in "accordance" with the approved guidelines. The study was approved by the National Agency for Medicine of Finland and the Ethics Committee of the local University Hospital (Helsinki University Central Hospital, Helsinki). Besides, a written informed consent was obtained from all the subjects after a detailed explanation of the procedures.

\section{Results}

Scopolamine significantly increased connectivity in the delta band and significantly reduced it in the alpha, beta and gamma band as compared to glycopyrrolate (placebo). Figure 14 shows the distribution of these changes across different cortical regions. Particularly, in the delta band scopolamine increased connectivity between the left parahippocampal and the right inferior occipital cortex. Additionally, we found reduced connectivity in the alpha band between the left superior frontal and the left angular cortex, the left superior temporal pole and the right precentral cortex, and between the right angular and the left lingual cortex. In the beta band, we found significantly reduced connectivity, specifically between the left medial superior frontal and the left superior parietal cortex, the left precuneus and the right lingual cortex, and between the left superior parietal and right fusiform cortex. Finally, in the gamma band, there was significantly reduced connectivity between the right middle frontal cortex and the left rolandic operculum, and between the right middle orbito-frontal cortex and the right precuneus. We also found reduced gamma band connectivity of the left inferior frontal operculum with the right lingual and left paracentral lobule.

As for the brain network indices, $C$ shows a significant reduction $(p<0.04)$ and $L$ a significant increased $(\mathrm{p}<0.04)$ both of them under scopolamine as compared to placebo in the alpha band, as depicted in Figure 15. 


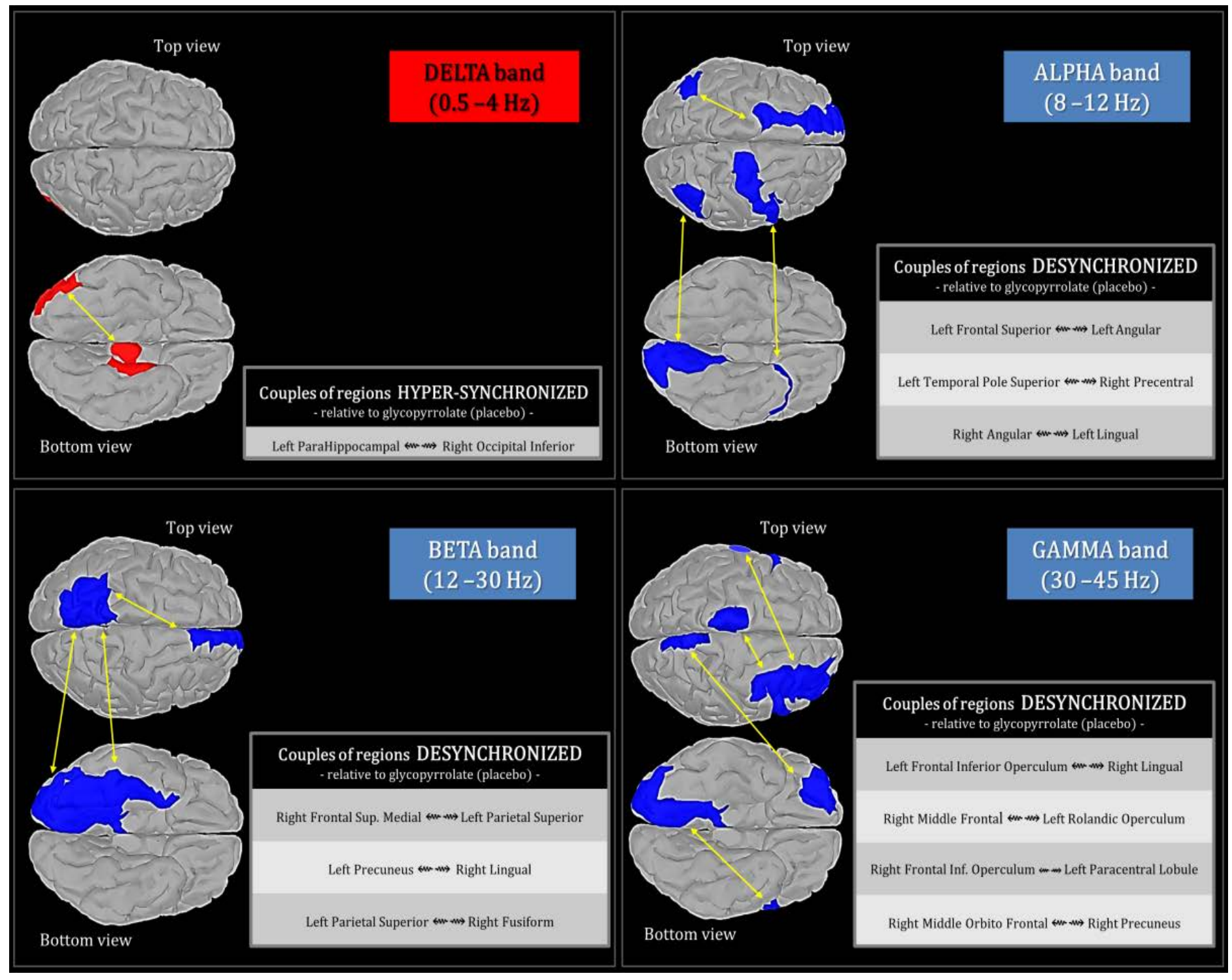

Figure 14. Significant differences in PLV between the Glycopyrrolate (placebo) and the scopolamine conditions for different frequency bands (at the p<0.005 level). In the delta band (top left) the synchronization between left parahippocampal and right occipital interior cortex increases under the scopolamine. On the contrary in the alpha (top right), beta (bottom left) and gamma bands (bottom right), the PLV decreases between different brain regions under scopolamine. 
NETWORK PARAMETERS (mean values: $C$ and $L$ )

\begin{tabular}{|c|cl|ll|}
\hline & \multicolumn{2}{|c|}{ Clustering (C) } & \multicolumn{2}{c|}{ Shortest path (L) } \\
\hline \multirow{2}{*}{ Delta } & $\mathrm{C}_{\text {placebo }}=0.197$ & $\mathrm{C}_{\text {scopolamine }}=0.194$ & $\mathrm{~L}_{\text {placebo }}=4.323$ & $\mathrm{~L}_{\text {scopolamine }}=4.253$ \\
\cline { 2 - 5 } Theta & $\mathrm{C}_{\text {placebo }}=0.209$ & $\mathrm{C}_{\text {scopolamine }}=0.181$ & $\mathrm{~L}_{\text {placebo }}=4.054$ & $\mathrm{~L}_{\text {scopolamine }}=4.607$ \\
\cline { 2 - 5 } Alpha & $\mathrm{C}_{\text {placebo }}=0.182$ & $\mathrm{C}_{\text {scopolamine }}=0.159$ & $\mathrm{~L}_{\text {placebo }}=4.307$ & $\mathrm{~L}_{\text {scopolamine }}=4.816$ \\
\cline { 2 - 5 } Beta & $\mathrm{C}_{\text {placebo }}=0.145$ & $\mathrm{C}_{\text {scopolamine }}=0.131$ & $\mathrm{~L}_{\text {placebo }}=4.812$ & $\mathrm{~L}_{\text {scopolamine }}=5.169$ \\
\hline Gamma & $\mathrm{C}_{\text {placebo }}=0.186$ & $\mathrm{C}_{\text {scopolamine }}=0.161$ & $\mathrm{~L}_{\text {placebo }}=4.426$ & $\mathrm{~L}_{\text {scopolamine }}=4.925$ \\
\hline
\end{tabular}

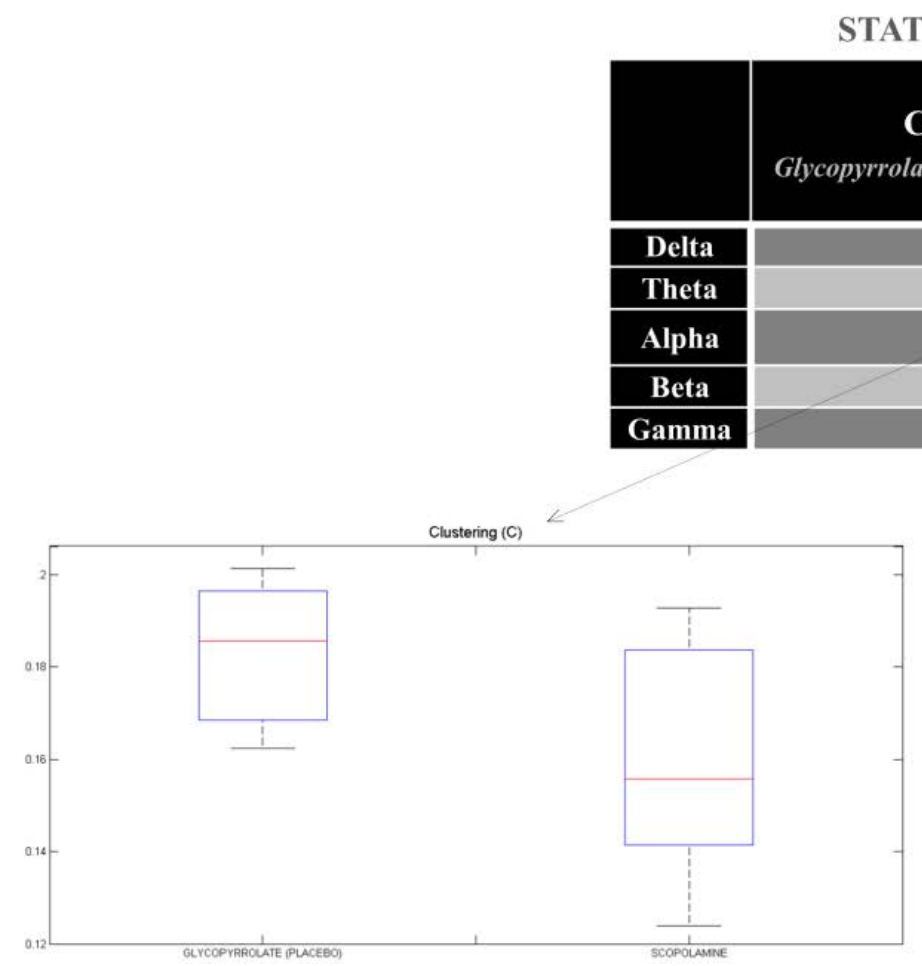

Clustering (C)

copyrrolate (placebo) vs. Scopolamine

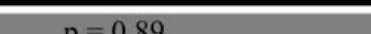

\begin{tabular}{|c|c}
\hline$p=0.89$ & $p=0.84$ \\
\hline$p=0.08$ & $p=0.13$ \\
\hline$p=0.04$ & $p=0.04$ \\
$p=0.11$ & $p=0.10$ \\
$p=0.08$ & $p=0.08$ \\
\hline
\end{tabular}

Shortest path (L)

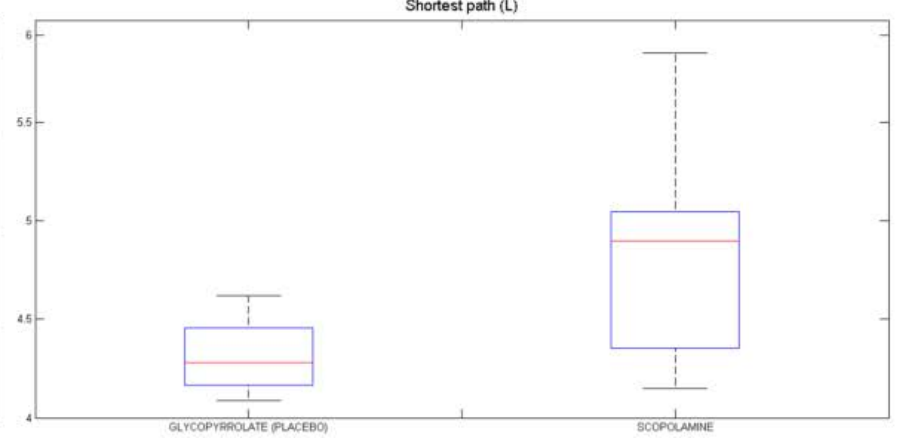

Figure 15. Both network parameters ( $C$ : clustering; $L$ : average shortest average path length) showed a significant reduction under scopolamine as compared to placebo in the alpha band. $(C: \mathrm{p}<0.042, L: \mathrm{p}<0.043)$. 


\section{Discussion}

We investigated here whether functional brain networks were impaired after the administration of a cholinergic receptor antagonist (scopolamine) in healthy elderly subjects. Additionally, we evaluated whether the effects of scopolamine on FC and functional MEG network structure mirrored the disconnection syndrome reported in AD patients ${ }^{136-138}$. Our findings showed that, compared to placebo, scopolamine significantly increases MEG phase synchronization in the delta band, specifically between the left medial temporal region (i.e. para-hippocampus) and the contralateral occipital cortex (taking into account the reservations, in terms of precision, which involves the calculation of such deep sources). This increment in slow band connectivity may reflect the excitotoxicity or pathological effect of scopolamine administration and was probably the result of the cholinergic cortical deafferentation from subcortical structures ${ }^{42,44,139}$. Scopolamine, however, decreased functional connectivity between several cortical regions in alpha, beta and gamma bands. In the alpha band, this reduction involved the left fronto-parietal and the inter-hemispheric fronto-temporal and parieto-occipital connectivity. Abnormalities in faster frequencies were characterized by reduced inter-hemispheric connections of the left parietal cortex (i.e. beta band) and the frontal lobes (i.e. gamma band). Using another index of phase synchronization, the so-called phase lag index (PLI), Stam et al136. reported that MEG resting-state $\mathrm{FC}$ was decreased in $\mathrm{AD}$ patients for frequencies above $8 \mathrm{~Hz}$. In fact, and consistent with our results, these authors found lower left fronto-parietal connectivity values in $\mathrm{AD}$ relative to healthy controls for the alpha band, as well as a disruption in fronto-temporal and parieto-occipital circuits. There was also a significant impairment of right parietal lobe connections. For faster frequencies (13-30 Hz), they also observed a predominant decrease in interhemispheric frontal connectivity and in frontoparietal circuits. Moreover, consistent with our outcomes, most studies hitherto have found decreased functional connectivity mainly in alpha and beta bands in AD patients compared to healthy controls ${ }^{42,140-143 .}$

Several cortical regions showing scopolamine-induced FC decrease in this study (e.g. precuneus, medial frontal and inferior parietal cortex) are part of the 
default mode network (DMN), which is known to be active during resting and attenuated during cognitive tasks. Specific regions of the DMN, including the precuneus in the medial parietal cortex, are selectively vulnerable to early pathological changes in $\mathrm{AD}^{144}$. Besides, precuneus was identified as a critical anatomical area ${ }^{145-147}$, thus suggesting that this structure could be a "network hub" which is affecting other functional regions. Beyond the DMN, other cortical areas showed loss of FC. For instance, we also found abnormal connectivity in areas of the anterior temporal network, including the hippocampus and the temporal pole ${ }^{148}$. Interestingly, this network is involved in memory function, which is typically impaired in $\mathrm{AD}^{144}$.

It is noteworthy that cortical areas that belong to other brain networks also exhibited disruption in FC after scopolamine administration. These networks include the fronto-parietal and the dorso-lateral prefrontal ones, which are involved in attention and executive functions, respectively. As mentioned above, Iturria-Medina et al.146 identified "the most indispensable and critical anatomical areas in the brain network", which among others also included: superior parietals and superior frontals. These regions, which were also disrupted by scopolamine administration are important brain network hubs. Besides, this finding is in line with that of a recent resting-state fMRI study ${ }^{148}$ reporting disrupted functional connectivity between multiple brain networks in AD. Taken together, these findings suggest that there is scopolamine-related disorganization of several cortical networks, where the DMN appears to be particularly compromised.

In addition to connectivity analysis in source space, we examined changes in the large-scale structure of resting-state functional brain networks using concepts from graph theory. In a recent review, Tijms et al. ${ }^{138}$ claim that the un-normalized path length (L) was most consistently reported (6 of 8 studies) to be increased in AD. This increase has been usually interpreted to result from the loss of connectivity. Moreover, regarding the clustering (C) index, these authors indicate that no definite differences have been found between both groups (AD and Controls), since the studies reviewed showed considerable variability. Hence, the present study is consistent with the findings reported the literature about the increase of path length in $\mathrm{AD}$, and supports the idea that the clustering index 
decreases in the lower alpha band in $\mathrm{AD}$ patients as compared to healthy subjects ${ }^{136}$.

Thus, what we have found here supports the idea that scopolamine may be considered as a psychopharmacological model of AD, since both the drug and the disease produce a similar disruption in FC and brain network architecture. Importantly, donepezil, a drug belonging to the family of cholinesterase inhibitors, has been reported to improve abnormalities in FC of the hippocampus and prefrontal areas ${ }^{149}$, which provides further support to this argument.

Overall, the present study shows that the pharmacological induction of amnesia in a group of healthy elderly subjects seems to produce a similar disruption of FC and functional MEG brain networks organization to that reported in AD patients. At any rate, some reservations must be pointed out before confirming that scopolamine administration produces similar alterations that those observed in $\mathrm{AD}$ patients. It is necessary to indicate that in the present study we did not perform any correlations between FC and the behavioral data while subjects were under scopolamine condition. In addition, we did not analyze an AD cohort but rather discuss our findings in the context of the existing literature of AD effects on functional MEG connectivity44,136,140,150. From these results, and considering the limitations discussed, we conclude that the assessment of functional MEG connectivity and the subsequent characterization of the brain network structure may be a useful approach to understand $\mathrm{AD}$, and provide new strong evidences of the usefulness of combining both methodologies to study brain function from multivariate neuroimaging data in health and disease. 


\section{Chapter 4}

\section{Network Disruption and Cerebrospinal Fluid Amyloid-Beta and Phospho-Tau Levels in Mild Cognitive Impairment ${ }^{2}$}

Synaptic dysfunction is a core deficit in AD, preceding hallmark pathological abnormalities. Resting-state MEG was used to assess whether functional connectivity patterns, as an index of synaptic dysfunction, are associated with CSF biomarkers [i.e., phospho-tau (p-tau) and amyloid beta (A $\beta-42)$ levels]. We studied 12 human subjects diagnosed with mild cognitive impairment due to Alzheimer's disease, comparing those with normal and abnormal CSF levels of the biomarkers. We also evaluated the association between aberrant functional connections and structural connectivity abnormalities, measured with diffusion tensor imaging, as well as the convergent impact of cognitive deficits and CSF variables on network disorganization. One-third of the patients converted to Alzheimer's disease during a follow-up period of 2.5 years. Patients with abnormal CSF p-tau and A $\beta-42$ levels exhibited both reduced and increased functional connectivity affecting limbic structures such as the anterior/posterior cingulate cortex, orbitofrontal cortex, and medial temporal areas in different frequency bands. A reduction in posterior cingulate functional connectivity mediated by p-tau was associated with impaired axonal integrity of the hippocampal cingulum. We noted that several connectivity abnormalities were predicted by CSF biomarkers and cognitive scores. These preliminary results indicate that CSF markers of amyloid deposition and neuronal injury in early Alzheimer's disease associate with a dual pattern of cortical network disruption, affecting key regions of the default mode network and the temporal cortex. MEG is useful to detect early synaptic dysfunction associated with AD brain pathology in terms of functional network organization.

2 The results presented in this chapter has been published in the following paper: S. Pusil, Canuet, L. et al. Network Disruption and Cerebrospinal Fluid Amyloid-Beta and Phospho-Tau Levels in Mild Cognitive Impairment. J. Neurosci. 35, 10325-10330 (2015). 


\section{Materials and Methods}

MEG recordings were obtained from 12 patients with MCI (mean age: 69.42 8.19; female: n 10), recruited from the Ramón y Cajal Hospital in Madrid, Spain. All of them were right-handed. MCI diagnosis was made according to the National Institute on Aging-Alzheimer Association (NIA-AA) clinical criteria151. In addition to clinical features, evidence of amyloid deposition and/or neuronal injury as indicated by CSF biomarkers was used to diagnose these patients with MCI due to Alzheimer's disease as intermediate or high likelihood and MCI converters. With regard to neuronal injury biomarkers, we focused on CSF p-tau protein because it is considered to be more specific to Alzheimer's-type dementia compared with CSF total tau ${ }^{53}$.

The levels of p-tau and $A \beta-42$ in the CSF were measured using ELISA following supplier recommendations (Innotest; Innogenetics). Cutoff points were obtained by our laboratory and sent to the Alzheimer's Association Quality Control Program Work Group blindly to clinical or neurophysiological data ${ }^{152}$. For each CSF biomarker, patients were divided into CSF-abnormal (biomarker positive) and CSF-normal (the biomarker-negative) group. Those with CSF levels of p-tau 60 $\mathrm{pg} / \mathrm{ml}$ and $\mathrm{A} \beta-42485 \mathrm{pg} / \mathrm{ml}$ made up the CSF-abnormal group for the corresponding biomarker. Otherwise, they were included in the CSF-normal group. Neuropsychological assessment included the Clinical Dementia Rate (CDR), the Mini Mental State Examination (MMSE), and the immediate and free recall tests of the 7 Minute Screening Neurocognitive Battery (7MS).

MEG recordings were acquired with a 306-channel Vectorview system (Elekta-Neuromag). Patients were in an awake, resting state with their eyes closed. For each subject, 5 min task-free data were recorded. The sampling frequency applied was $1000 \mathrm{~Hz}$. Recordings were filtered offline and corrected for head movements with a temporal signal space separation with movement compensation (tsss-mc) method (Maxfilter 2.2 software). Analyses were performed for alpha (8 $12 \mathrm{~Hz}$ )-, beta (12-30 Hz)-, and gamma (30 - $45 \mathrm{~Hz}$ )-frequency bands based on evidence for a role of alpha-band connectivity in the diagnosis of $\mathrm{MCI}^{46,56}$ and the significance of alpha, beta, and gamma-frequency bands to resting-state global network function ${ }^{153}$. 
The scalp and cortex surface were extracted from each subject's MRI volumes and imported into Brainstorm (http://neuroimage.usc.edu/ brainstorm), the open-source software used for source reconstruction and connectivity analysis $^{129}$. The cortex surface was divided into 88 regions of interest (ROIs) based on the Brainstorm default atlas. The source activity for each subject and frequency band was computed using the weighted minimum norm estimation with the default settings on Brainstorm. For the cortex, the source activity obtained was projected onto the default anatomy of Brainstorm. After source map projection onto the default anatomy, the time series of cortical ROIs were extracted.

As in the study described in the previous chapter, we used here the phaselocking value (PLV) to assess FC between all pairs of regions (88 x 88) for each frequency band ${ }^{126}$. Phase synchronization measures are based on the hypothesis that the difference of phases between two phase-locked systems must be nonuniform, so the degree of nonuniformity must be a good estimator of the coupling level. For analysis, first, data were band-pass filtered using a zero-phase distortion finite impulse response filter with a bandwidth of $2 \mathrm{~Hz}$. Then, the complex analytic signal from each of the brain regions was obtained by using the Hilbert transform (for details, see Pereda et $\mathrm{al}^{121}$ ). Subsequently, the PLV between the time series $x(k)$ and $y(k)$ from two brain regions is defined as mentioned before in equation (22).

Hippocampal volumes were measured as anatomical evidence of the degree of brain atrophy that characterizes MCI51. A high-resolution T1-weighted MRI was acquired for each subject with a Philips 1.5 T MRI scanner using a fast-field echo sequence. Freesurfer software (version 5.1.0) and its specialized tool for automated subcortical segmentation were used to segment the subject's T1weighted volume into different regions. Finally, hippocampal volume was normalized with the overall intracranial volume to account for differences in head volume over subjects.

Diffusion tensor images (DTIs) were obtained using a linear least-squares approach in FSL-FDT and several scalar images representing the shape of the diffusion tensor were obtained: fractional anisotropy, mean diffusivity, radial diffusivity, and axial diffusivity. Images with b $0 \mathrm{~s} / \mathrm{mm} 2$ (i.e., b0 images) were 
realigned to brain extracted T1-weighted images using an affine linear transformation in FSL-FLIRT, with mutual information as cost function and nearest neighbor interpolation. Linear affine transformations followed by nonlinear local deformations using FSL-FNIRT were applied to normalize brain-extracted T1weighted images into the MNI brain template of $1 \mathrm{~mm} 3$ isotropic. Transformations were concatenated and applied inversely to transform the JHU white matter tractography atlas ${ }^{154}$ into the diffusion subject-specific space. The tracts of interest included the anterior thalamic radiation, corticospinal tract, cingulum, hippocampal cingulum, forceps major and minor, inferior fronto-occipital fasciculus, superior longitudinal fasciculus, inferior longitudinal fasciculus, and uncinate fasciculus.

For statistical analyses, based on the non-Gaussian distribution of the MEG data, connectivity results were compared between the CSF-abnormal and CSF-normal group for each biomarker using the Mann Whitney U test. A total of 5000 permutations were used to correct for multiple comparisons. The neuropsychological scores were correlated with the values (PLVs) of the functional connections found significantly different between groups and with the CSF levels of each biomarker using Spearman correlation coefficient (uncorrected). For an integrative approach of the impact of neuropsychological scores and CSF variables on neural function, multivariate regression models were built, with these variables acting as predictors and the functional connections as the dependent variables. For DTI analysis, average values within the 20 atlas tracts for the scalar images were obtained and statistical group comparisons were performed using an unpaired $\mathrm{t}$ test. The correlation between PLV and the fractional anisotropy values of the significantly different tracts was measured using Spearman correlation coefficient (uncorrected). 


\section{Results}

Demographic and clinical characteristics of the patients are shown in Table 1. There were no differences in age, cognitive scores, or hippocampal volumes across groups. Abnormal CSF p-tau and $A \beta-42$ levels were found in seven and eight patients, respectively. Five of them were positive for both biomarkers at the time of the study. Four MCI patients converted to Alzheimer's disease during the followup period of 2.5 years. These patients showed low levels of $A \beta-42$ and high levels of $\mathrm{p}$-tau in the CSF.

Table 1. Patients' characteristics. Data are mean \pm standard deviation unless otherwise noted.

\begin{tabular}{|c|c|c|c|c|c|c|}
\hline & \multicolumn{3}{|l|}{ Phospho-Tau } & \multicolumn{3}{|c|}{ Amyloid Beta (1-42) } \\
\hline & $\begin{array}{l}\text { Pathological } \\
\quad(n=7)\end{array}$ & $\begin{array}{c}\text { Non- } \\
\text { pathological } \\
(n=5) \\
\end{array}$ & $\begin{array}{c}p- \\
\text { values }\end{array}$ & $\begin{array}{l}\text { Pathological } \\
\quad(n=8)\end{array}$ & $\begin{array}{c}\text { Non- } \\
\text { pathological } \\
(n=4) \\
\end{array}$ & $\begin{array}{c}p- \\
\text { values }\end{array}$ \\
\hline Age & $69.86 \pm 6.51$ & $\begin{array}{c}68.80 \pm \\
10.96\end{array}$ & 0.85 & $66.50 \pm 7.03$ & $75.25 \pm 7.93$ & 0.12 \\
\hline MMSE score & $26 \pm 1.09$ & $27.80 \pm 2.49$ & 0.19 & $27.57 \pm 1.99$ & $25.50 \pm 1.29$ & 0.07 \\
\hline Immediate & & & & & & \\
\hline Recall & $4.83 \pm 3.31$ & $5.0 \pm 1.87$ & 0.92 & $5.29 \pm 3.09$ & $4.25 \pm 1.71$ & 0.49 \\
\hline Free Recall & $12.17 \pm 5.67$ & $14.20 \pm 2.19$ & 0.45 & $12.71 \pm 5.28$ & $13.75 \pm 2.63$ & 0.67 \\
\hline $\begin{array}{c}\text { Hippocampal } \\
\text { volume Left }\end{array}$ & $\begin{array}{l}0.0021 \pm \\
0.00042\end{array}$ & $\begin{array}{l}0.0024 \pm \\
0.00032\end{array}$ & 0.11 & $\begin{array}{l}0.0024 \pm \\
0.00045\end{array}$ & $\begin{array}{l}0.0021 \pm \\
0.00033\end{array}$ & 0.32 \\
\hline $\begin{array}{l}\text { Hippocampal } \\
\text { volume Right }\end{array}$ & $\begin{array}{l}0.002 \pm \\
0.00041\end{array}$ & $\begin{array}{l}0.0024 \pm \\
0.00027\end{array}$ & 0.11 & $\begin{array}{l}0.0022 \pm \\
0.00041\end{array}$ & $\begin{array}{l}0.0019 \pm \\
0.00027\end{array}$ & 0.18 \\
\hline
\end{tabular}




\section{p-tau}

Patients with abnormal CSF p-tau levels showed an abnormal pattern characterized by decreased FC between the right posterior cingulate cortex and paracentral lobule in alpha band, the right orbitofrontal cortex and contralateral calcarine area in the beta band, and the right paracentral lobule and lingual cortex in the gamma band. Increased connectivity was observed between the right supplementary motor area and the contralateral cuneus in the alpha band. In addition, there was increased intrahemispheric connectivity between the right anterior cingulate cortex and medial temporal area in the beta band and between the right middle frontal cortex and anterior temporal area in the gamma band (Figure 16, Table 2).

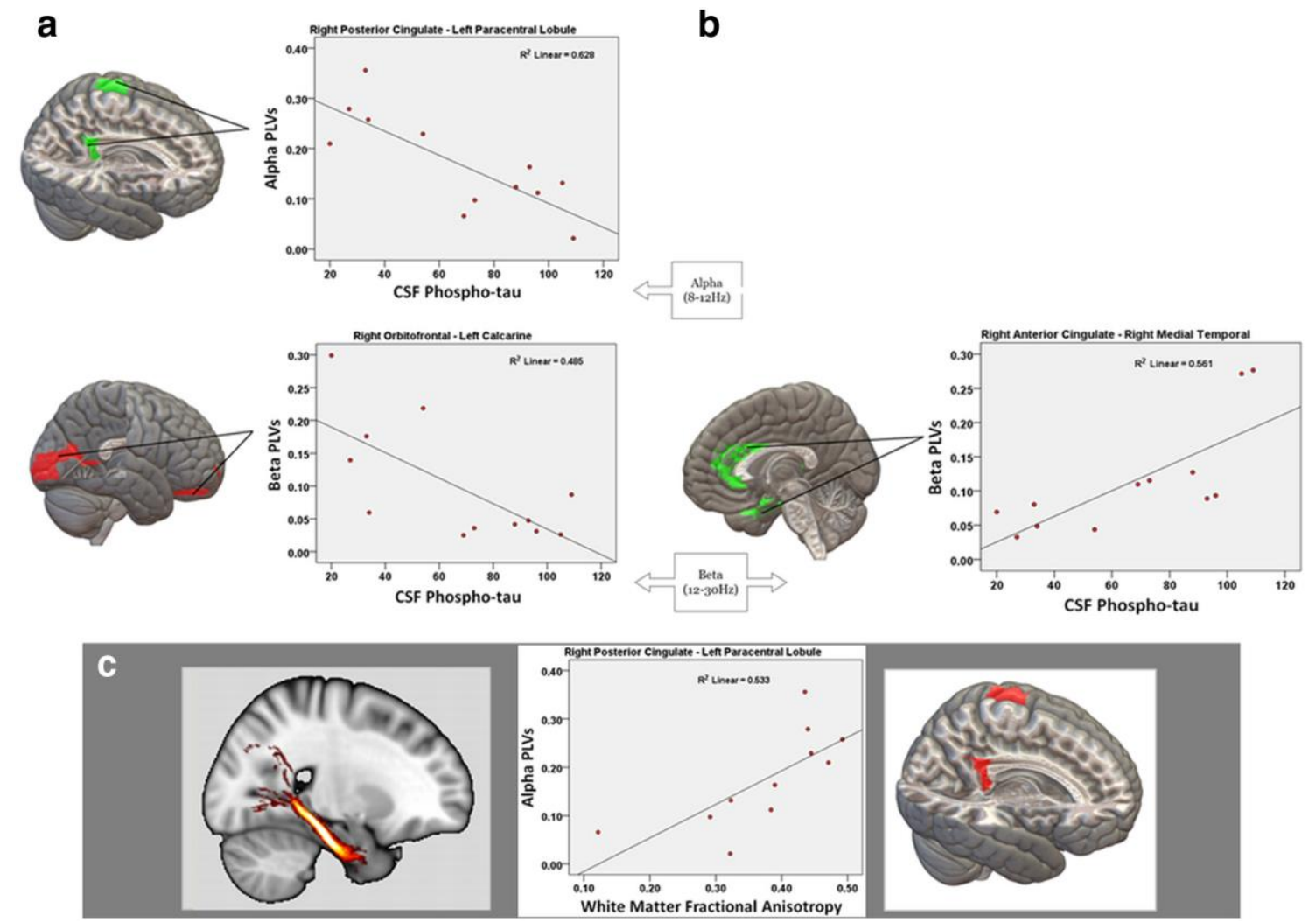

Figure 16. Pairs of regions showing significant functional connectivity abnormalities predicted by p-tau levels in the CSF. Cortical regions show p-tau-related desynchronization $(\boldsymbol{a})$ and p-tau-related hypersynchronization $(\boldsymbol{b})$. Scatterplots of the correlations between these functional connections and p-tau levels are provided. $\boldsymbol{c}$, Significant correlation between functional and structural connections. DTIs of the right hippocampal cingulum and the desynchronized regions show a significant correlation with fractional anisotropy. Scatterplots of the correlation between fractional anisotropy and connectivity values are provided. 
Table 2. Phase synchronization and correlation values of cortical regions showing significantly different functional connectivity across biomarker groups

\begin{tabular}{|c|c|c|c|c|c|c|c|c|c|}
\hline \multirow[b]{2}{*}{ Frequency bands } & \multirow[b]{2}{*}{ Pairs of regions } & \multicolumn{2}{|c|}{ MCl groups mean connectivity } & \multicolumn{2}{|c|}{$\begin{array}{l}\text { Connectivity } \\
\text { (statistics) }\end{array}$} & \multicolumn{4}{|c|}{ Multivariate regression } \\
\hline & & p-tau abnormal & p-tau normal & $x^{2}$ & $p$-value & Model's adj. $R^{2}$ & Predictor & $F$ & p-value \\
\hline \multirow[t]{2}{*}{ Alpha $(8-12 \mathrm{~Hz})$} & Posterior cingulate (R) and paracentral lobule (L) & 0.10 & 0.21 & 5.55 & 0.018 & 0.64 & CSF p-tau & 9.42 & 0.028 \\
\hline & Cuneus $(\mathrm{L})$ and supplementary motor cortex (R) & 0.12 & 0.06 & 8.06 & 0.004 & 0.28 & - & - & - \\
\hline \multirow[t]{3}{*}{ Beta $(12-30 \mathrm{~Hz})$} & Orbitofrontal cortex $(\mathrm{R})$ and calcarine $(\mathrm{L})$ & 0.04 & 0.18 & 7.18 & 0.007 & 0.79 & CSF p-tau & 25.97 & 0.004 \\
\hline & Anterior cingulate (R) and medial temporal cortex $(\mathrm{R})$ & 0.15 & 0.05 & 8.08 & 0.004 & 0.76 & Free recall & 9.96 & 0.025 \\
\hline & & & & & & & CSF p-tau & 9.21 & 0.029 \\
\hline \multirow[t]{3}{*}{ Gamma $(30-45 \mathrm{~Hz})$} & Paracentral lobule (R) and lingual (L) & 0.05 & 0.14 & 7.10 & 0.008 & 0.53 & - & - & - \\
\hline & Middle frontal cortex (R) and anterior temporal (R) & 0.14 & 0.04 & 8.08 & 0.004 & 0.25 & - & - & - \\
\hline & & $A \beta 42$ abnormal & $A \beta 42$ normal & & & & & & \\
\hline \multirow[t]{2}{*}{ Alpha $(8-12 \mathrm{~Hz})$} & Posterior cingulate (R) and middle temporal cortex (R) & 0.13 & 0.32 & 7.31 & 0.006 & 0.14 & - & - & - \\
\hline & Medial temporal cortex (R) and superior parietal cortex (L) & 0.18 & 0.30 & 6.49 & 0.010 & 0.64 & CSF $A \beta 42$ & 8.71 & 0.032 \\
\hline \multirow[t]{3}{*}{ Beta $(12-30 \mathrm{~Hz})$} & Inferior temporal cortex (R) and precentral (R) & 0.04 & 0.16 & 7.38 & 0.006 & 0.85 & MMSE & 7.24 & 0.040 \\
\hline & & & & & & & CSFA $\beta 42$ & 41.22 & 0.001 \\
\hline & Medial prefrontal cortex (R) and fusiform (R) & 0.13 & 0.06 & 6.40 & 0.011 & 0.68 & CSF $A \beta 42$ & 14.76 & 0.012 \\
\hline \multirow[t]{2}{*}{ Gamma $(30-45 \mathrm{~Hz})$} & Inferior temporal cortex (R) and precentral (R) & 0.08 & 0.18 & 7.30 & 0.006 & 0.84 & CSFA $\beta 42$ & 21.45 & 0.006 \\
\hline & Superior temporal (R) and anterior temporal (L) & 0.05 & 0.13 & 6.41 & 0.011 & 0.67 & CSFA $\beta 42$ & 22.30 & 0.005 \\
\hline
\end{tabular}

\section{A $\beta-42$}

Patients with abnormal CSF $A \beta-42$ levels exhibits decreased functional connectivity between right temporal areas and several cortical regions of the frontal, parietal, and temporal lobes compared with patients with normal CSF levels. We also found decreased alpha FC between the right posterior cingulate cortex and the ipsilateral middle temporal cortex, as well as between the right medial temporal and left superior parietal cortex. In the beta band, there was decreased FC between the right inferior temporal cortex and the ipsilateral precentral area. These areas also exhibited decreased connectivity in the gamma band. In addition, a decrease in gamma connectivity was found between the right superior temporal cortex and the contralateral anterior temporal area. Abnormal CSF A $\beta-42$ levels were also linked to a hyperconnectivity pattern. There was an increase in functional connectivity of the right medial-superior frontal cortex with the right fusiform area in the beta band (Figure 17, Table 2). 

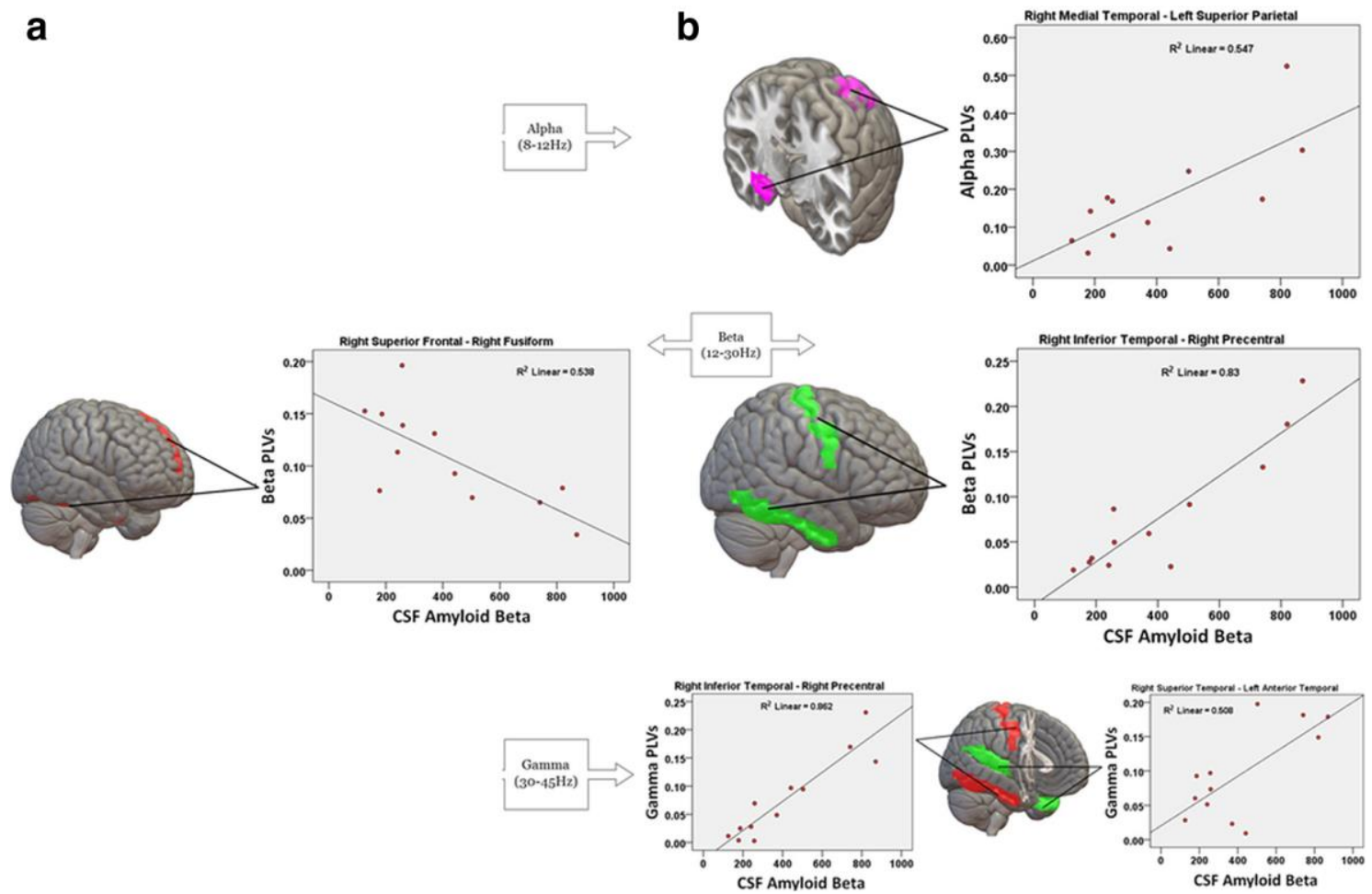

Figure 17. Pairs of regions showing significant functional connectivity abnormalities predicted by $A \beta-42$ levels in the CSF. Cortical regions show $A \beta-42$-related hypersynchronization (a) and $A \beta-42$ related desynchronization (b). Scatterplots of the correlations between these functional connections and $\mathrm{A} \beta-42$ levels are provided.

\section{Cognitive scores}

An increase in beta connectivity between the right anterior cingulate cortex and the ipsilateral medial temporal cortex showed a negative correlation with the MMSE score $(r=0.79 ; p=0.003)$ and the free recall score $(r=0.67 ; p=0.023)$ in patients with abnormal CSF p-tau levels relative to those with normal levels. In addition, a decrease in interhemispheric connectivity between the right posterior cingulate cortex and paracentral area correlated positively with the immediate recall score $(r=0.72 ; p=0.012)$. In the $C S F$-abnormal $A \beta-42$ group, a positive correlation was found between the MMSE score and decreased beta connectivity between the inferior temporal and precentral cortex $(r=0.77 ; p=0.005)$. A correlation analysis of cognitive scores and CSF biomarkers revealed that only the MMSE score correlated with the CSF levels of both $p$-tau $(r=0.68 ; p=0.021)$ and $A \beta$ 42 ( $\mathrm{r}=0.82 ; \mathrm{p}=0.002)$. To explore the convergent impact of cognitive scores and 
CSF values on brain functional connectivity, we performed multivariate regression analysis with the significant connections as the dependent variables (Table 2). We found that the CSF levels of the biomarkers predicted most of the aberrant functional connections seen in these MCI patients. In addition, the free recall score showed a predictive value for intrahemispheric anterior cingulate-medial temporal connectivity, whereas the MMSE score predicted functional connectivity between the inferior temporal and precentral cortex.

\section{Correlation between FC and structural connectivity}

The correlation analysis of the significant functional connections and structural connectivity measures revealed that the decrease in alpha synchronization between the right posterior cingulate and left paracentral lobule mediated by p-tau correlated positively with the fractional anisotropy value of the right hippocampal cingulum ( $\mathrm{r}=0.81 ; \mathrm{p}=0.003)$. This fractional anisotropy reduction correlated with CSF $p$-tau levels $(r=0.70 ; p=0.016)$. There was no significant correlation between fractional anisotropy and cognitive scores

\section{Discussion}

In this study, we assessed whether functional connectivity pat-terns were associated with CSF biomarkers (i.e., p-tau, and A $\beta-42$ concentrations) in patients with MCI due to AD.

In addition, we evaluated the relationship of the aberrant functional connections with structural connectivity abnormalities and cognitive deficits. Patients with p-tau pathology had reduced functional connectivity affecting limbic structures such as the posterior cingulate and the orbitofrontal cortex, as well as medial parietal areas, in particular the paracentral lobule (Figure 16). CSF p-tau levels predicted abnormalities specifically in the alpha- and beta-frequency range (Figure 16, Table 2). It is well established that tau hyperphosphorylation is associated with intracellular aggregation of neurofibrillary tangles and Alzheimer's-disease-related neurodegeneration ${ }^{52,155}$. Recent evidence suggests 
that, even in the preclinical stage of Alzheimer's disease, limbic structures are affected by tau pathology. There is also evidence that limbic dysfunction involves primarily the entorhinal cortex and then spreads to the parietal cortex ${ }^{156}$ and inferior frontal areas, including the orbitofrontal cortex ${ }^{157}$. This phenomenon is thought to be due to synaptic loss and trans-synaptic spread of pathological forms of tau through brain circuits involved in learning and memory formation ${ }^{158}$, which can explain the typical cognitive deficits seen in MCI due to AD.

Interestingly, the reduced functional connectivity of the posterior cingulate cortex mediated by p-tau correlated positively with impaired axonal integrity of the ipsilateral hippocampal cingulum, as indicated by a significant decrease in fractional anisotropy (Figure 16c). This supports the notion that the disruption of anatomical networks influences brain organization at the functional level, resulting in the manifestations of MCI syndrome ${ }^{58}$. Consistent with this finding, an investigation performed by Amlien et al. ${ }^{159}$, in which CSF bio-markers were also used, revealed regional white matter loss in the right hippocampal cingulum in association with tau pathology. Together, these results and ours suggest a strong relationship among synaptic dysfunction, axonal disintegration in limbic areas, and tau pathology as key pathophysiological mechanisms involved in MCI due to Alzheimer's disease.

In addition to decreased functional connectivity, patients with abnormal CSF p-tau levels also showed a hyperconnectivity pattern affecting mainly the anterior cingulate and medial temporal cortex (Figure 17b), which was predicted by the free recall score (Table 2). Therefore, this increase in connectivity affecting the anterior cingulate cortex may reflect a pathological activity that exerts a negative impact on cognition, particularly on memory function, and may increase the risk of conversion to Alzheimer's disease ${ }^{46}$.

A reduction in CSF A $\beta$-42 levels was associated with decreased functional connectivity of the posterior cingulate cortex and neo-cortical temporal regions. The lateral parietal and medial temporal regions were also involved. Disrupted connectivity of the inferior temporal cortex in the beta band was predicted by the MMSE score. The posterior cingulate and lateral parietal cortex are key regions of the default mode network, a brain circuit typically active during rest, the 
structures of which are vulnerable to $A \beta$ deposition ${ }^{160,161}$. Consistent with our findings, fMRI and PET studies demonstrated that $A \beta$ plaques disrupt resting-state connectivity within the default mode network ${ }^{160,162}$ and in the lateral temporal cortex, including inferior temporal areas ${ }^{163}$, in preclinical Alzheimer's disease and MCI.

We also noted a decrease in connectivity of the medial temporal cortex associated with the $A \beta-42$ biomarker. This cortical area is thought to have a low $A \beta$ burden, particularly in early stages of the disease ${ }^{157}$. However, the spatial distribution of $A \beta$ deposition and its effect on hippocampus function during the course of dementia is still a matter of debate. Therefore, although a direct toxic effect of $A \beta$ plaques on medial temporal structures cannot be ruled out completely, this decrease in connectivity could be mediated by connectional diaschisis ${ }^{164}$. This phenomenon explains that the intimate anatomo-functional relation between regions showing amyloid accumulation and the hippocampus might affect the ability of this area to establish close communication with other brain regions, resulting in connectivity reduction and consequently in a reduced neuronal input from those regions. Consistent with this assumption, a recent report using CSF biomarkers also suggested an association between decreased CSF A $\beta-42$ and decreases in fMRI functional connectivity in the posterior cingulate and medial temporal cortex in MCI and Alzheimer's disease ${ }^{165}$.

We found that $A \beta$-related hypersynchronization in this study was characterized by the involvement of prefrontal connections. Because this abnormality showed no association with cognitive deficits and the prefrontal cortex is thought not to be affected early in Alzheimer's-type dementia, this increased connectivity might be linked to a compensatory mechanism. This would be in agreement with evidence indicating that patients with $\mathrm{AD}$ use additional neural resources in prefrontal cortex, presumably those mediating executive functions, to compensate for losses attributable to the degenerative process of the disease ${ }^{166}$.

Overall, our results should be considered as preliminary based on the small sample size of MCI patients. Nevertheless, this study indicates that CSF abnormal levels of p-tau and $A \beta-42$ in early $A D$ are associated with cortical network disruption 
involving desynchronization and hypersynchronization of key regions of the default mode network; for example, the anterior and posterior cingulate cortex and specific temporal and frontal areas. This dual pattern could represent two sides of a disrupting network that loses equilibrium, leading to cognitive impairment. Upon confirmation of these findings, MEG may potentially be used as a tool to detect early synaptic dysfunction associated with Alzheimer's disease brain pathology in terms of functional network organization. 


\section{Chapter 5}

\section{On the role of resting state MEG FC in longitudinal studies of MCI patients}

The recent inclusion of biomarkers in the diagnostic criteria for AD by the National Institute on Aging-Alzheimer's Disease Association (NIA-AA), such as A $\beta$ deposition or neuronal injury, has been considered an important advance in characterizing "in vivo" the disease ${ }^{167}$. In addition, these biomarkers contribute for the consideration of the AD as a continuum of clinical and biological phenomena. For this reason, the NIA-AA has recently proposed three consecutive phases of the disease: preclinical, symptomatic pre-dementia MCI and AD dementia 51,167,168. However, the question of which people without dementia will finally develop the disorder remains unanswered. A reasonable explanation for this phenomenon is to consider that during the course of the disease, these pathophysiological and cognitive changes are not as linear as has been contemplated 169 .

One of the most studied phases in the prognosis of AD is MCI, since it entails a higher risk of developing Alzheimer- type dementia170,171. However, the progression of this transitional stage between normal aging and dementia has usually been quite variable due to its heterogeneous nature. The inclusion of more precise clinical criteria and biomarker's characteristics is being decisive in getting a diagnosis and prognosis increasingly accurate. Therefore, those MCI patients meeting the clinical criteria and pathological evidence for AD are now referred as "MCI due to AD", with different levels of certainty ${ }^{51 .}$

Most related studies have analyzed the role of cognitive tests in predicting the conversion from MCI to $\mathrm{AD}$, the performance in episodic memory being the best predictor ${ }^{172,173}$. A greater atrophy in hippocampal volume and entorhinal cortex has been also found in those MCI subjects who finally progressed to $\mathrm{AD}^{46,174,175}$. Fluorodeoxyglucose positron emission tomography (FGD-PET) studies have shown that progressive MCI subjects (pMCI) presented a lower regional metabolism over temporal, parietal, and/or precuneus cortices compared to those MCI patients that remained stable during the same period of time (stable MCI, sMCI) ${ }^{176}$. Pittsburgh compound B (PIB)-PET investigations have concluded that 
PIB- positive MCI subjects exhibited a higher risk of progression to AD than those PIB- negative ${ }^{177}$. In this line, studies of $A \beta$ deposition in CSF have described that pMCI subjects exhibited lower levels than sMCI subjects 178,179 , while the inverse pattern has been identified for the tau protein 178 .

Other possible indicators, as neurophysiological ones, have not yet been considered as markers of the disease. However, EEG and specially MEG may provide relevant information in the progression of AD. For instance, in a recent MEG study of our group, pMCI subjects showed higher FC in alpha band between the right anterior cingulate and temporo-occipital regions than sMCI subjects. Furthermore, this hypersynchronization was related with a worse cognitive status and a higher atrophy of temporal lobe structures ${ }^{46}$.

A remarkable amount of prospective studies have focused on the progression from MCI to $\mathrm{AD}$, but there is a scarcity of longitudinal investigations that have used repeated measurements of the subjects over time, and none of them made use of MEG. In the present work, we studied a sample of MCIs that were followed-up during a 3-years period, being their magnetic signals measured twice over this time, one at the beginning and one at the end. We used FC and EC as applied to MEG to study the evolution from MCI to AD and the functional network alterations due to the progression of the disease. In addition, we developed a model based on FC and neuropsychological scores to predict the conversion to AD. 


\section{Materials and Methods}

\section{Subjects}

For the present study, we recruited fifty-four MCI subjects from the Hospital Universitario San Carlos (Madrid, Spain). They were all right handed ${ }^{180}$ native Spanish speakers. In Table 3 we present their demographic and clinical data.

All participants were screened by means of standardized diagnostic instruments and received an exhaustive neuropsychological assessment as previously described in López et al ${ }^{181}$. The MCI diagnosis was established according to the National Institute on Aging- Alzheimer Association (NIA-AA) criteria $^{51}$, which includes: (i) self- or informant-reported cognitive complaints; (ii) objective evidence of impairment in one or more cognitive domains; (iii) preserved independence in functional abilities; and (iv) not demented ${ }^{167}$. Besides meeting the clinical criteria, MCI participants had signs of neuronal injury (hippocampal volume measured by magnetic resonance imaging (MRI). So, they might be

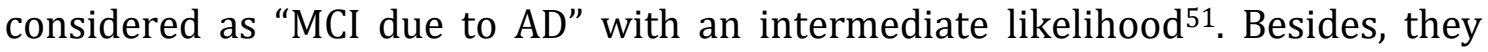
were cognitively and clinically followed-up during three years (every six months) and were then split into two groups according to their outcome: 1) the "progressive" MCI group (pMCI; $n=27$ ) was composed of those subjects that met the criteria for probable $\mathrm{AD}^{167}$ and 2) the "stable" MCI group (sMCI; $\mathrm{n}=27$ ) was comprised of those participants that still fulfilled the diagnosis criteria of MCI at the end of follow-up.

None of the participants had a history of psychiatric or neurological disorders (other than MCI or AD). General inclusion criteria were: age between 65 and 80, a modified Hachinski score $\leq 4$, a short-form Geriatric Depression Scale score $\leq 5$, and T1 magnetic resonance imaging (MRI) within 12 months and 2 weeks before the two MEG recordings without indication of infection, infarction, or focal lesions (rated by two independent experienced radiologists; Bai et al ${ }^{182}$ ). Patients were off those medications that could affect MEG activity, such as cholinesterase inhibitors, $48 \mathrm{~h}$ before recordings. 
The study was approved by the Hospital Universitario San Carlos Ethics Committee (Madrid), and all participants signed a written informed consent prior to participation.

\begin{tabular}{lcccc}
\hline & sMCI (n=27) & pMCI (n=27) & F-value & p-value \\
\hline Age (years) & $71,23 \pm 3,98$ & $74,81 \pm 3,98$ & 2,6137 & $0,009^{*}$ \\
Gender & 15 & 18 & Fisher test & 0,577 \\
Apoe & 12 & 13 & Fisher test & 0,782 \\
Education(years) & $8,88 \pm 4,49$ & $8,6 \pm 4,49$ & 0,0064 & 0,937 \\
MMSE score & $27,34 \pm 3,39$ & $25,95 \pm 3,39$ & 3,2289 & 0,079 \\
MMSE (post-condition) & $26,19 \pm 4,13$ & $23,65 \pm 4,13$ & 2,9490 & 0,092 \\
Direct digit spam & $6,84 \pm 2,28$ & $7 \pm \pm 2,28$ & 0,6555 & 0,422 \\
Inverse digit spam & $4,46 \pm 1,33$ & $4,23 \pm 1,33$ & 0,1765 & 0,676 \\
Immediate recall & $19,34 \pm 8,58$ & $11,76 \pm 8,58$ & 5,5128 & $0,023^{*}$ \\
Delayed recall & $7,58 \pm 4,98$ & $2,76 \pm 4,98$ & 3,8756 & 0,055 \\
Rule shift cards & $2,16 \pm 1,34$ & $1,84 \pm 1,34$ & 0,0143 & 0,905 \\
VOSP & $6,83 \pm 2,78$ & $6,46 \pm 2,78$ & 0,0742 & 0,787 \\
Phonemic fluency & $8,2 \pm 4,25$ & $8,88 \pm 4,25$ & 0,1792 & 0,674 \\
Semantic fluency & $11,94 \pm 3,99$ & $11,96 \pm 3,99$ & 0,4430 & 0,509 \\
TMTA (time) & $82,86 \pm 46,69$ & $94,36 \pm 46,69$ & 0,6593 & 0,421 \\
TMTB (time) & $227,35 \pm 127,84$ & $254,95 \pm 127,84$ & 0,0194 & 0,890 \\
Ideomotor praxis & $7,29 \pm 0,83$ & $7,30 \pm 0,83$ & 0,4103 & 0,525 \\
BNT & $45,8 \pm 9,01$ & $44,65 \pm 9,01$ & 0,0730 & 0,788 \\
BNT (phonemic) & $6,33 \pm 2,83$ & $6,11 \pm 2,83$ & 0,0425 & 0,838 \\
Hippocampal volume Left & $0,0024 \pm 0,0003$ & $0,002 \pm 0,0003$ & 9,7773 & $0,003^{*}$ \\
Hippocampal volume Right & $0,0025 \pm 0,0003$ & $0,0022 \pm 0,0003$ & 5,5714 & $0,023^{*}$ \\
Entorhinal volume Left & $0,0014 \pm 0,0005$ & $0,0012 \pm 0,0005$ & 1,1795 & 0,284 \\
Entorhinal volume Right & $0,0013 \pm 0,0003$ & $0,0013 \pm 0,0003$ & 1,7137 & 0,197 \\
Parahippocampal volume Left & $0,0013 \pm 0,0002$ & $0,0013 \pm 0,0002$ & 1,1296 & 0,294 \\
Parahippocampal volume Right & $0,0013 \pm 0,0001$ & $0,0012 \pm 0,0001$ & 4,6403 & $0,037^{*}$ \\
\hline
\end{tabular}

Table 3. Mean \pm SD values of the demographic and clinical characteristics of the sMCI and pMCI patients. MMSE, Mini Mental State Examination; BNT, Boston Naming Test; VOSP, Visual Object and Space Perception Battery; TMTA, Trail-Making Test Part A; TMTB, Trail-Making Test Part B.

$p$-values for between-groups differences were introduced, and ${ }^{*} \mathrm{p}<0.05$. Age differences were assessed with a Mann-Whitney Test. An ANCOA test, with age as a co-variable, was used for continuous variables and Fisher's exact test for gender and APOE differences.

\section{MRI and Hippocampal volumes}

3D T1 weighted anatomical brain magnetic resonance imaging (MRI) scans were collected with a General Electric 1.5T MRI scanner, using a high-resolution antenna and a homogenization PURE filter (Fast Spoiled Gradient Echo (FSPGR) 
sequence with parameters: TR/TE/TI $=11.2 / 4.2 / 450 \mathrm{~ms}$; flip angle $12^{\circ} ; 1 \mathrm{~mm}$ slice thickness, a $256 \times 256$ matrix and FOV $25 \mathrm{~cm}$ ).

We employed Freesurfer software (version 5.1.0.21) to obtain the hippocampal volumes, which were normalized with the overall intracranial volume to account for differences in head volume over subjects.

\section{MEG recordings}

MEG signals were acquired using a whole-head Elekta-Neuromag MEG system with 306 channels (Elekta AB, Stockholm, Sweden) at the Center for Biomedical Technology (Madrid, Spain). Data was collected at a sampling frequency of $1000 \mathrm{~Hz}$ and online band-pass filtered between 0.1 and $330 \mathrm{~Hz}$.

MEG recordings were obtained at the same time of the day in two different moments: 1) at baseline (pre-condition), and 2) three years later (post-condition). The MEG protocol consisted of 5 min of resting-state with eyes closed. Subjects were seated comfortably inside of a magnetically shielded room and were asked to relax and to reduce as much as possible body movements. Four coils were placed in the subject head to monitor the head position and to co-register with the MRI later. Also, landmarks like nasion and left and right pre-auricular were digitized using a Polemus Fastrak digitizer. Recordings were filtered offline and corrected for head movements with a temporal signal space separation with movement compensation method (Maxfilter 2.2 software).

Artifacts such as eye movements, blinks, muscular activity and jumps were detected by an automatic algorithm within the Fieldtrip toolbox ${ }^{183}$ and also visually inspected by a MEG expert. Artifacts generated by heartbeats were removed using an ICA algorithm. Clean data was segmented in 4s length epochs. For subsequent analysis, we used only the data from the magnetometers.

\section{Source Reconstruction and Connectivity Analysis}

The scalp and cortex surface were extracted from each subject's MRI volumes using Freesurfer. Also, a single shell model was computed as the forward 
modeling of MEG measures using the Fieldtrip toolbox ${ }^{183}$. Analyses were performed in the following frequency bands: theta $(4-8 \mathrm{~Hz})$, alpha $(8-12 \mathrm{~Hz})$, beta $(12-30 \mathrm{~Hz})$ and gamma $(30-45 \mathrm{~Hz})$. Then, source activity was computed in a grid of neural sources, uniformly distributed, of approx. $1 \mathrm{~cm}^{2}$ using Linearly Constrained Minimum Variance beamformer ${ }^{184}$ individually for each subject and frequency band. This neural MEG sources where anatomically parcellated by dividing the cortex into 90 regions according to the AAL atlas ${ }^{128}$. We selected the PCA as the representative time series for each brain area. Finally, to find brain FC patterns we computed two different phase synchrony (PS) indices.

The first one, which estimates undirected $\mathrm{FC}$, is the phase locking value $(\mathrm{PLV})^{126}$, already used in the two previous studies of this thesis, which is defined as mentioned earlier (Eq. (22)).

The second PS index is a recently derived, directed one termed phase transfer entropy (PhTE) ${ }^{185}$. PhTE measures directed coupling strength of neuronal oscillations between time-series using their phases, and is based on the principle of Transfer Entropy (TE) ${ }^{186}$. TE is a model-free implementation of Wiener's principle of observational causality which states that for two simultaneously observed processes $\mathrm{X}, \mathrm{Y}$, we call X "causal" to $\mathrm{Y}$ if knowledge about the past of $\mathrm{X}$ improves our prediction of $Y$ over and above what is predictable from the past of $Y$ alone $^{120}$. TE quantifies the amount of predictive information actually transferred between two processes $\mathrm{X}$ and $\mathrm{Y}$ :

$$
T E_{x y}=\sum p\left(Y_{t+\partial}, Y_{t}, X_{t}\right) \log \left(\frac{p\left(Y_{t+\partial} \mid Y_{t}, X_{t}\right)}{p\left(Y_{t+\partial} \mid Y_{t}\right)}\right)
$$

Lobier et al ${ }^{185}$ defined the PhTE based on the instantaneous phase $\Phi$ of time-series $\mathrm{X}$ and $\mathrm{Y}$ :

$$
P h T E_{x y}=\sum p\left(\Phi_{Y_{t+\delta}}, \Phi_{Y_{t}}, \Phi_{X_{t}}\right) \log \left(\frac{p\left(\Phi_{Y_{t+\delta}} \mid \Phi_{Y_{t}}, \Phi_{X_{t}}\right.}{p\left(\Phi_{Y_{t+\delta}} \mid \Phi_{Y_{t}}\right)}\right)
$$


the prediction delay $\delta$ was set as the period corresponding to the mean frequency within each band, as we found it is the value giving rise to a maximum of PhTE (Appendix A).

The PhTE can also be expressed as a combination of entropies ${ }^{185}$ :

$$
\operatorname{PhTE}(\delta, X, Y)=H(\delta, X)+H(X, Y)-H(X)-H(\delta, X, Y)
$$

where $\mathrm{H}$ is the Shannon entropy and $\delta$, as in (24), is the future of $\mathrm{X}$. This entropy can be calculated using histograms. In this study, for each pair of regions, we used the normalized version of the PhTE as defined by ${ }^{187}$ :

$$
P h T E_{x y}=\frac{P h T E_{x y}}{P h T E_{x y}+P h T E_{y x}}
$$

The value of $P h T E_{x y}$ ranges between zero and one. When information flows from $\mathrm{X}$ to $\mathrm{Y}: 0.5<P h T E_{x y} \leq 1$. When information flows towards $\mathrm{X}$ from Y: $0 \leq P h T E_{x y}$ $<0.5$. If there is no preferential direction of information flow, $P h T E_{x y}=0.5$.

\section{Network Analysis}

As already commented in earlier chapters, another interesting approach to study the interaction between brain regions based on the connectivity matrices, is the use of graph theory ${ }^{188-191}$. Thus, in this section, we studied the network topology and some others network characteristics based on the PLV and PhTE.

First, for the PLV, we studied the participation coefficient which measures how evenly a node is connected to different modules of a network, capturing the distribution of the links of a node ${ }^{192}$. This is a network measure of inter-module connectivity.

The participation coefficient measures the distribution of a node's edges among the communities of a network. If the links of a node are entirely restricted to its community, its participation coefficient is zero. If the links of a node are evenly distributed among all communities, the participation coefficient approaches one. Hubs with low participation coefficients are called "provincial" hubs because 
their links are not distributed widely among communities, while hubs with higher participation coefficients are called "connector" hubs ${ }^{193}$. The participation coefficient for a node $i$ belonging to a module $m$ in a network with $M$ total modules is defined as ${ }^{192}$ :

$$
p c_{i}=1-\sum_{m=1}^{M}\left(\frac{k_{i, m}}{k_{i}}\right)^{2}
$$

The term $k_{i, m}$ denotes the within module degree, i.e., the number of links between node $i$ and other nodes within module $m$, and $\frac{k_{i, m}}{k_{i}}$ indicates the ratio of links a node has within its own module.

On the other hand, as mentioned in chapter 3 (methods), any graph $\mathrm{G}=(\mathrm{N}$, L) can be seen as a set of $\mathrm{N}$ nodes connected through L links. If the links have direction, the graphs are known as directed, as opposed to undirected ones, in which the FC index (e.g. PLV) provides no information about the directionality. In case of directed graphs, the number of links going into (resp. coming out) a node is its in-degree (resp. out-degree).

We studied the results from the PhTE from the perspective of graph theory. Adjacency matrices were built based on PhTE values greater than 0.5 that were set to one (zero otherwise). With these matrices, using Brain Connectivity toolbox ${ }^{133}$ (http://www.brain-connectivity-toolbox.net) we studied the in- and out degree in the pre-condition for both groups. The idea was to describe the network organization from a qualitative point of view.

Finally, to characterize the heterogeneity of the connectivity patterns in a multilayer MEG network, we computed the multiple participation coefficient (MPC) ${ }^{194}$. The multilayer network is composed of four layers corresponding to each of the four frequency-specific connectivity PLV matrices. Thus, the MEG multilayer network, built for each subject, consisted of $\mathrm{N}(\mathrm{N}=90)$ nodes and $\mathrm{M}$ $(\mathrm{M}=4)$ weighted layers $\lambda$.

The MPC is the multilayer version of the local participation coefficient in Eq (27). In the case of the multilayer network, it quantifies the participation of each 
brain region in the four MEG frequency-specific layers. It is based on the weighted degree of each node. For node $i$, MPC is defined as ${ }^{195}$ :

$$
M P C_{i}=\frac{M}{M-1}\left\lceil 1-\sum_{\lambda}\left(N L P_{i}^{[\lambda]}\right)^{2}\right\rceil
$$

where $N L P_{i}^{[\lambda]}=\frac{s_{i}^{\lambda}}{o_{i}}$, stands for node-weighted degree layer proportion, which measures the percentage of the total number of links of node $i$ that are in layer $\lambda$ $(\lambda=1, \ldots, 4)$. Nodes with high MPC may be considered as hubs of the multilayer network, because they allow the exchanging of information among different layers $^{196}$ (in this case, they are brain areas in which different frequencies are all heavily synchronized). The MPC ranges between 0 (the nodes tend to concentrate their connectivity in one layer) and 1 (the nodes tend to have the same number of links in every layer).

One important topic we have purposely not addressed hitherto is that of topological network filtering or pruning, also known in the literature as thresholding195,197-200. It refers to the (yet) unsolved problem of which links to include and which to discard when estimating a network measure. In the previous chapters, we have adopted the simplest approach, which consists of taking all the PLV values no matter how small and estimate the measures using their weighted versions. But here, in order to understand the possible role of thresholding on the results we obtain, we decided to use the recently derived method of the orthogonal minimal spanning trees (OMST) ${ }^{200}$ as well as the whole weighted PLV matrix for posterior comparisons. OMST is a topological thresholding technique with a novel proposed data-driven scheme, which attempts to maximize the information flow over the network vs. the cost by selecting the connections. Briefy, OMST is based on the notion of sampling the full-weighted brain matrix over consecutive rounds of minimum spanning trees (MST) ${ }^{201}$ that are orthogonal to each other. With this iterative approach, orthogonal MSTs and topologically filtering brain networks are obtained by optimizing the global efficiency of the network constrained by the cost $^{200}$, an idea similar to that of Fallani et al ${ }^{195}$, but presenting the advantage of guaranteeing a fully connected network. 


\section{Statistical analyses}

The PLV (post-condition/pre-condition) ratio was calculated to assess the ratio of change between the two stages of the longitudinal study. The procedure for statistical comparisons relied on the cluster based permutation test (CBPT) introduced by Maris and Oostenveld ${ }^{202}$. It was carried out independently for each frequency band, and goes as follows:

1-. We assess the FC pair-wise differences for each pair of ROIs using Ancova-test with age as a confounding covariate. Only those links with p-values < 0.05 were kept and included in the following step of the analysis. This step generates a robust significant connected subnetwork, also called a motif in graph theory ${ }^{203}$, which consisted of several connected links, which systematically showed differences in FC between the two MCI groups. Only motifs involving 7 or more ROIs ( $10 \%$ of the ROIs) were considered.

2.- Then, to control for multiple comparisons, a proper null distribution of F-values was estimated by randomizing 5000 times the original data. First, the group's configuration (maintaining the same number of subjects in each group) was randomized. Next, the FC matrices were shuffled. In each randomized dataset, the whole procedure was repeated, ending up with new surrogate motifs. Motifsstatistics were assessed through the sum of all F-values corresponding with the elements that composed the motif (F-values obtained in the node-level comparisons). The maximum statistic at each repetition was kept for the permutation distribution. The CBPT p-value represents the proportion of the permutation distribution with F-values greater or equal than the F-value of the original data. Only motifs with $\mathrm{p}<0.05$ were kept as significant motifs. Finally, the average (across all links that belong to the motif) FC values of these motifs (i.e. their corresponding strength) were used in the correlation analysis with the neuropsychological scores and grey matter volumes using Pearson correlation coefficient analysis. Statistical analyses were carried out using Matlab R2016a (Mathworks Inc). All tests were two-tailed.

Finally, for statistically significant PLV connectivity results, a classification analysis was performed using the hippocampal volumes and neuropsychological scores available for both groups with a logistic regression analysis with the leave- 
one-out cross-validation procedure. Leave-one-out is a special case of crossvalidation, which has shown to give an almost unbiased estimator of the generalization properties of statistical models, and therefore provides a sensible criterion for model selection and comparison. In this case, the algorithm picks only one point as the test set. Then, it builds a model on all the remaining, complementary points, and evaluates its error on the single-point held out. A generalization error estimate is obtained by repeating this procedure for each of the training points available, averaging the results ${ }^{204,205}$.

Leave-one-out can be defined using the least-squares polynomial regression:

$$
C V_{(n)}=\frac{1}{n} \sum_{n=1}^{n}\left(\frac{y_{i}-\widehat{y}_{l}}{1-h_{i}}\right)^{2}
$$

where $y_{i}$ is the actual value of the training point $i, \widehat{y}_{l}$ is the value predicted by the cross-validation model trained on all points except $i$. The $h_{i}$ is the diagonal element of the hat matrix. The hat matrix is the operator matrix that produces the least squares fit. This is also known as the self-influence. It is a measure of how much observation $i$ contributes to its own fit.

Results are described in terms of accuracy, sensitivity, specificity, positive predictive value (PPV), and negative predictive value (NPV). Accuracy represents the fraction of subjects classified correctly. Sensitivity and specificity represent the fractions of pMCI and sMCI patients correctly classified, respectively. Finally, PPVs and NPVs represent the fraction of patients classified as pMCI that were really pMCI and the fraction of patients classified as sMCI that were really sMCI, respectively. When reporting accuracy values, the information is completed with a confidence interval (CI) for the statistic, calculated using the B approach ${ }^{206}$.

For the PhTE analysis, we performed a t-test to compare the PhTE values with a distribution of mean equal to 0.5 to find the links with statistically significant directionality. 


\section{Results}

We only obtained statistically significant clusters $(\mathrm{p}<0.005)$ in theta and beta bands. In theta band, we found a decrease in the PLV ratio for the pMCI as compared to sMCI (Figure 18). The cluster included especially interhemispheric connections in fronto-temporal, fronto-occipital and hippocampus with frontal and occipital cortices. Additionally, for the beta band, the fronto-temporal and frontooccipital cortices exhibited a decrease in the PLV ratio for the pMCI compared to sMCI (Figure 19).
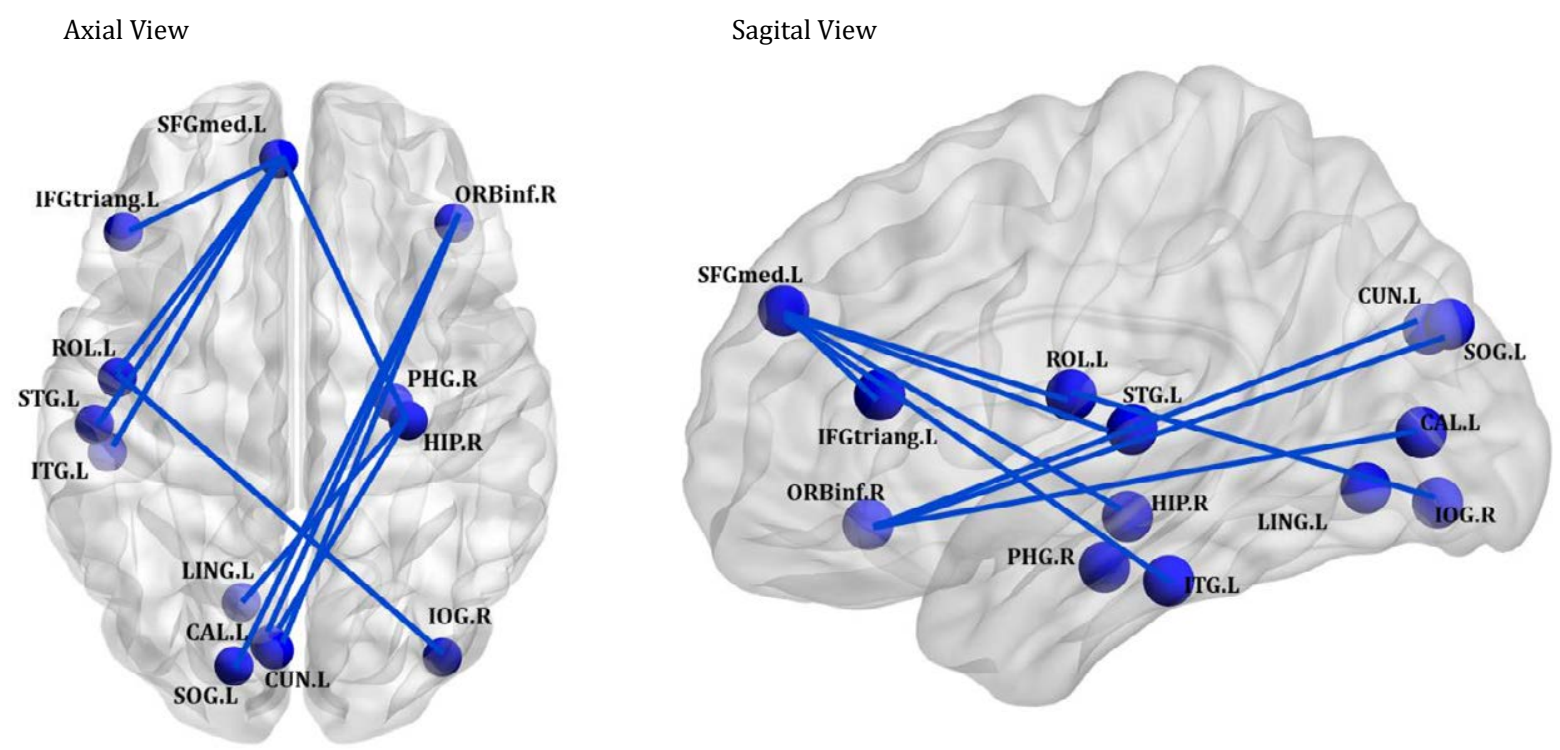

Figure 18. PLV ratio post/pre-condition in theta band $(\mathrm{p}<0.005)$. Blue links indicate $\mathrm{pMCI}<\mathrm{sMCI}$

Axial View

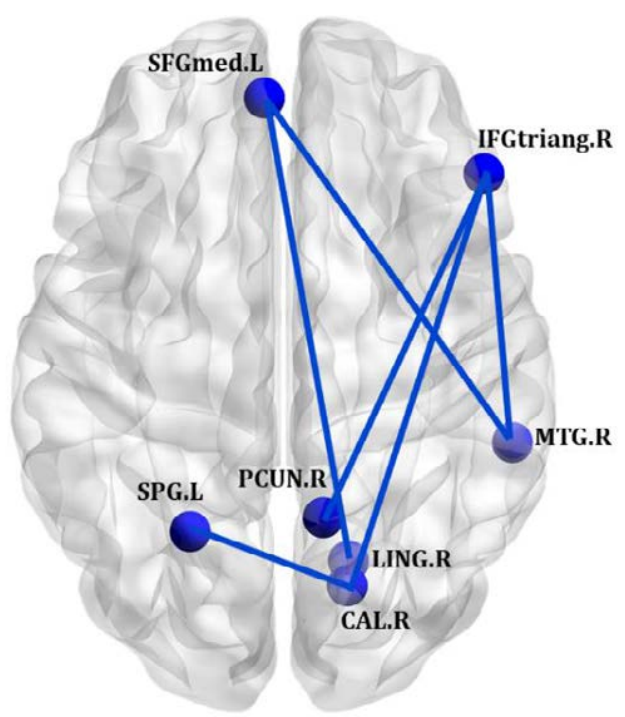

Sagital View

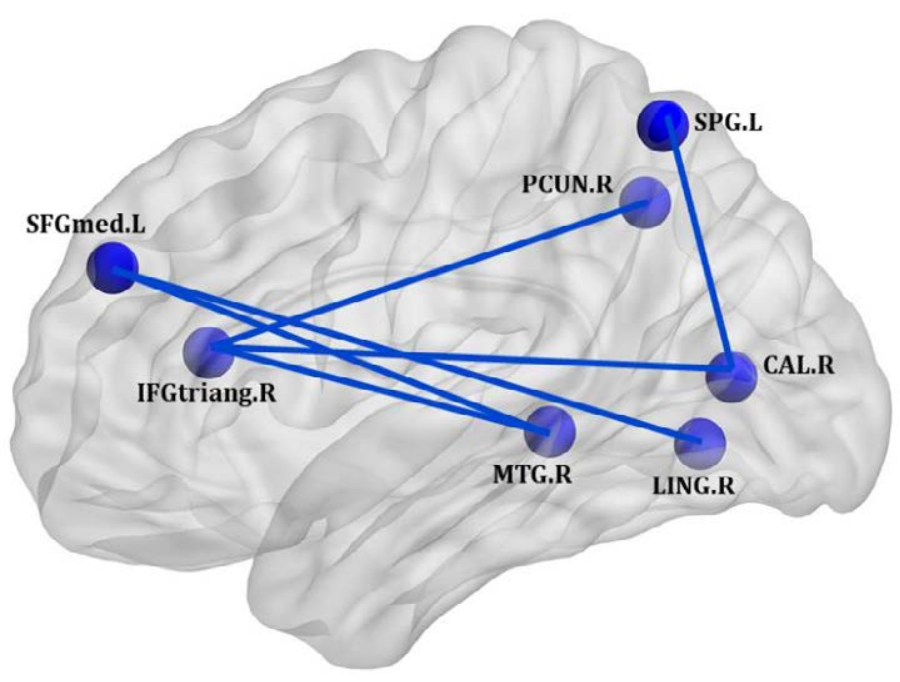

Figure 19. PLV ratio post/pre-condition in beta band $(\mathrm{p}<0.005)$. Blue links indicate $\mathrm{pMCI}<\mathrm{sMCI}$ 


\section{Correlation between FC and hippocampal volumes}

Figure 20A shows the correlation between the left hippocampus volume and the FC average cluster in theta band $(\mathrm{p}<0.017)$. Additionally, in beta band (Figure 20 $\mathrm{B} \& \mathrm{C})$, the right and the left hippocampus correlated positively with the $\mathrm{FC}$ average cluster ( $\mathrm{p}<0.014$ and $\mathrm{p}<0.026$ respectively).
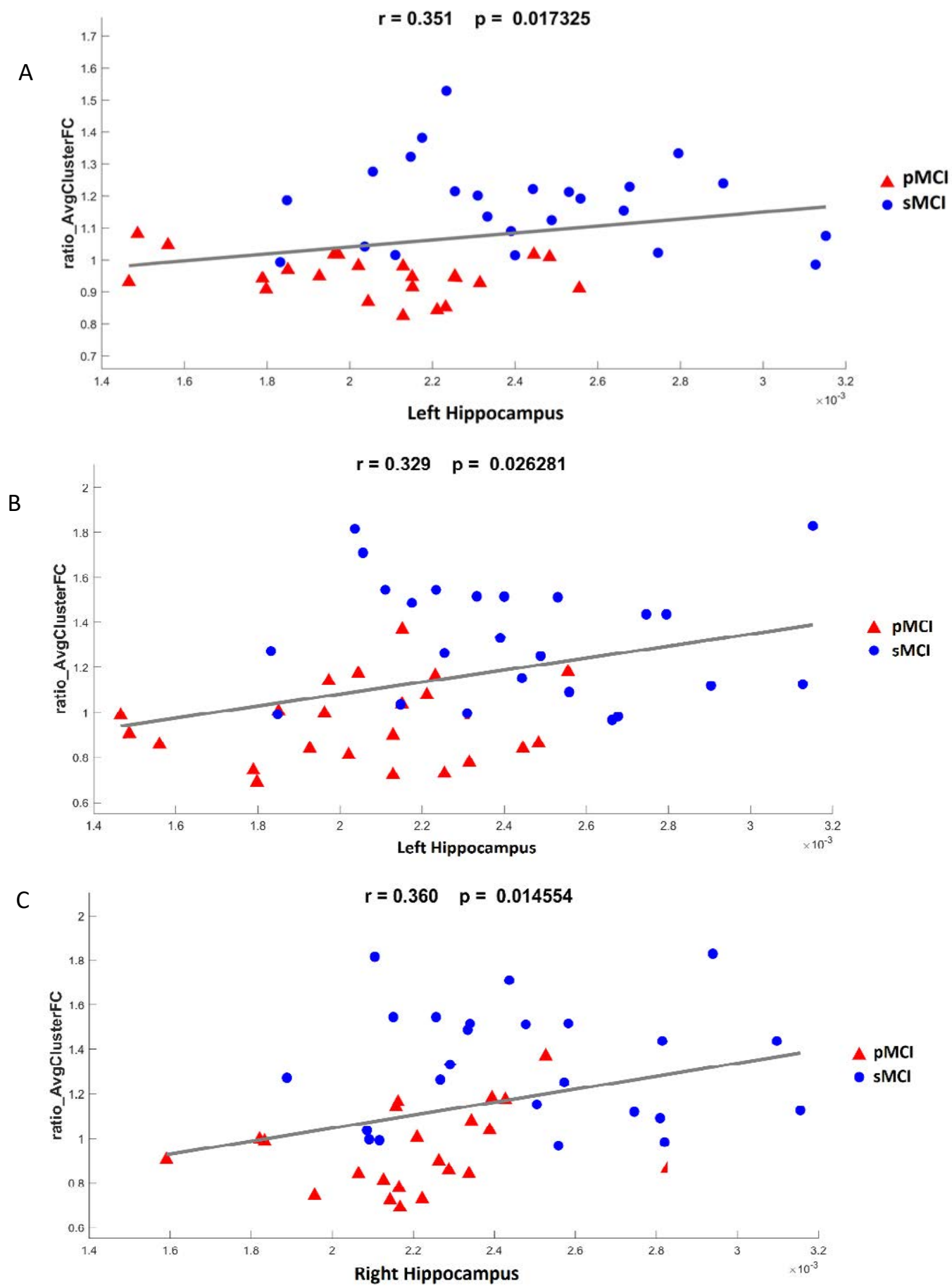

Figure 20. A. Correlations between the average PLV ratio of the significant clusters and the left hippocampal volumes in theta band. B. and C.: The same but for the left and right hippocampal volumes in beta band respectively. 


\section{Correlation between significant PLV ratio with neuropsychological scores}

To assess the possible functional meaning of this pattern of connectivity clusters in relation to cognitive functions, we performed a series of correlation analyses. In the theta band, immediate $(\mathrm{p}<0.014)$ and delay recall $(\mathrm{p}<0.007)$ correlated positively with the average PLV ratio of the significant cluster (see Figure 21).
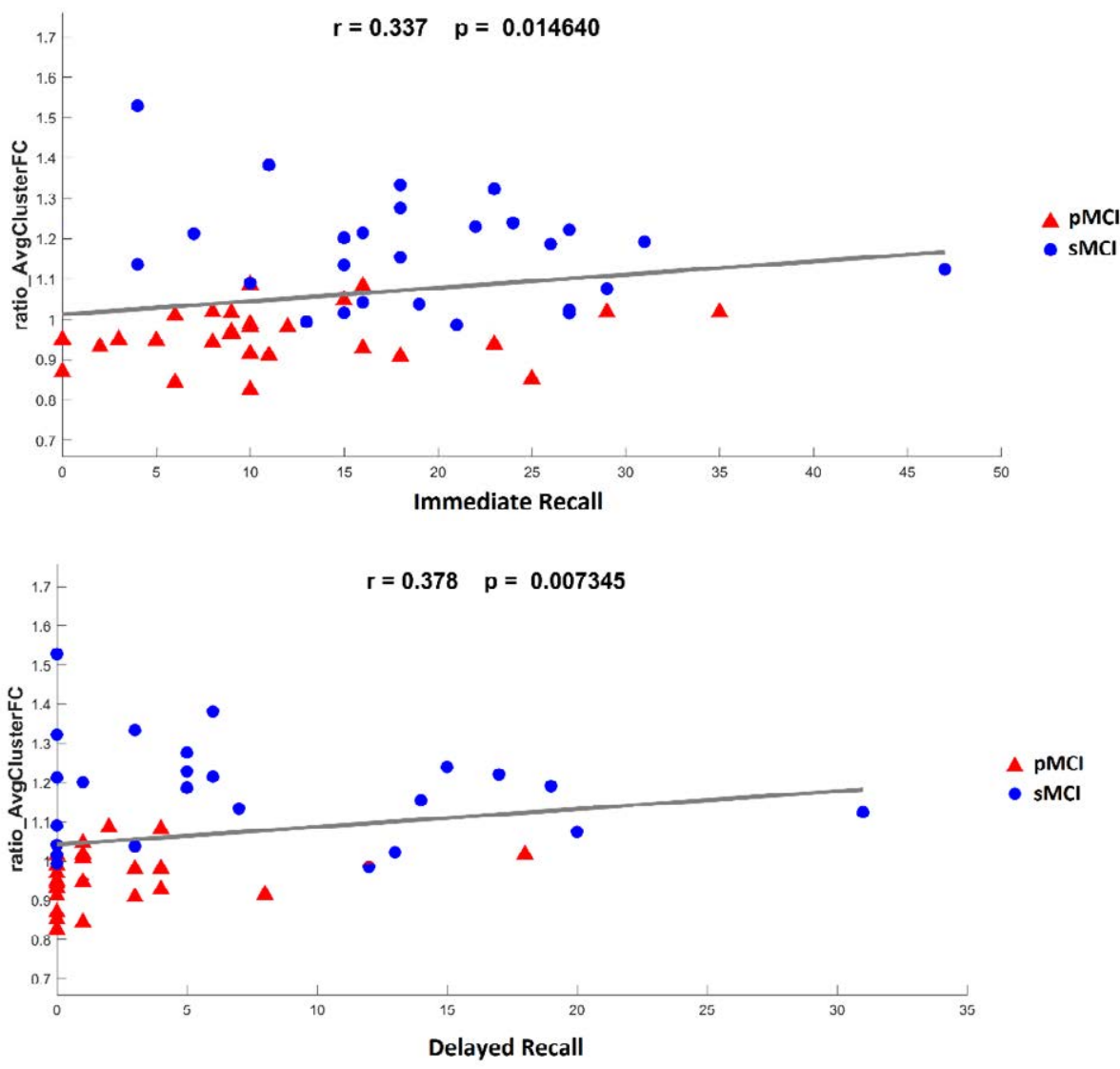

Figure 21. Correlations between PLV ratio and neuropsychological scores related with memory in theta band. 


\section{Groups evolution in pre- and post-conditions.}
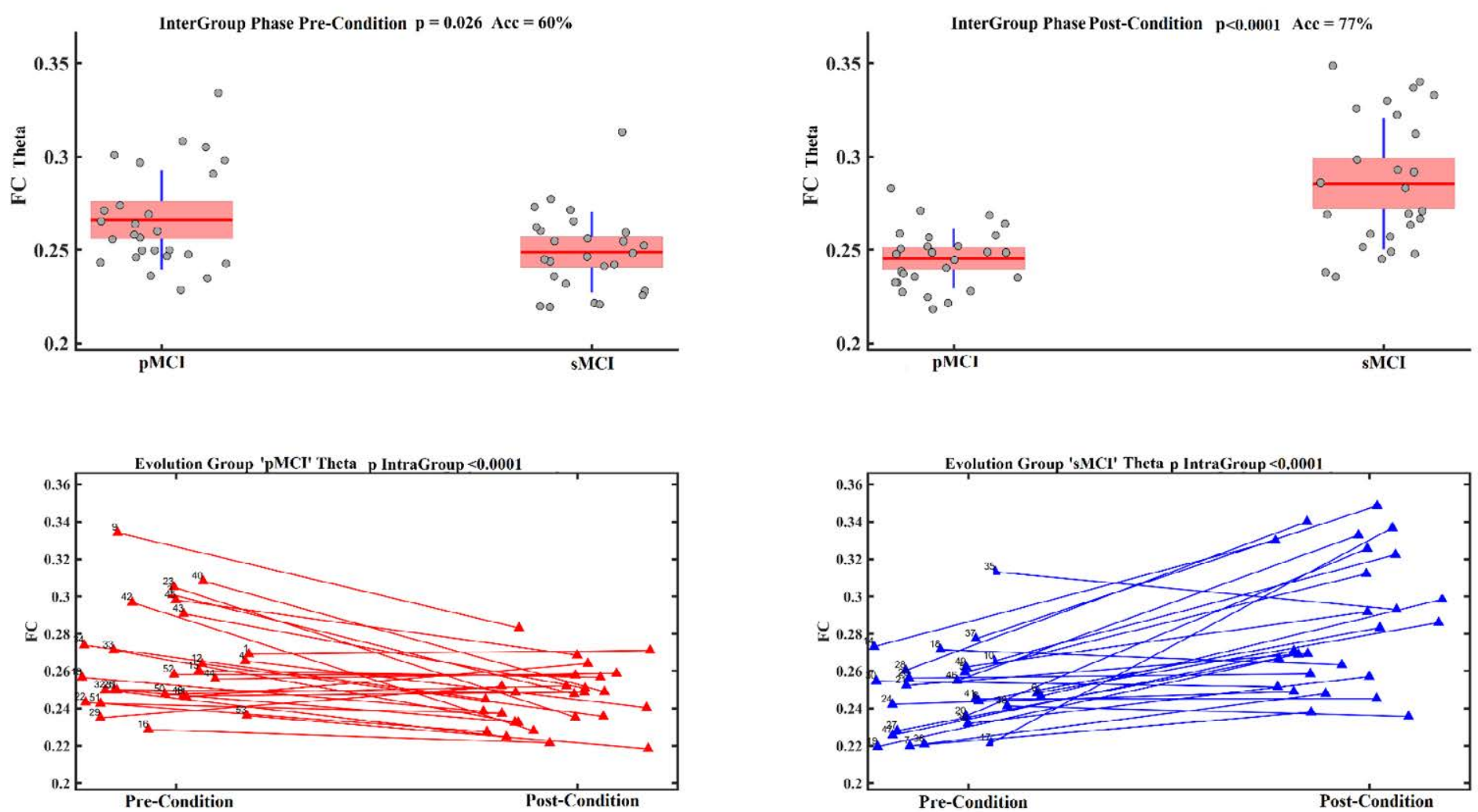

Figure 22. Evolution of each patient within each group in both conditions in theta band.
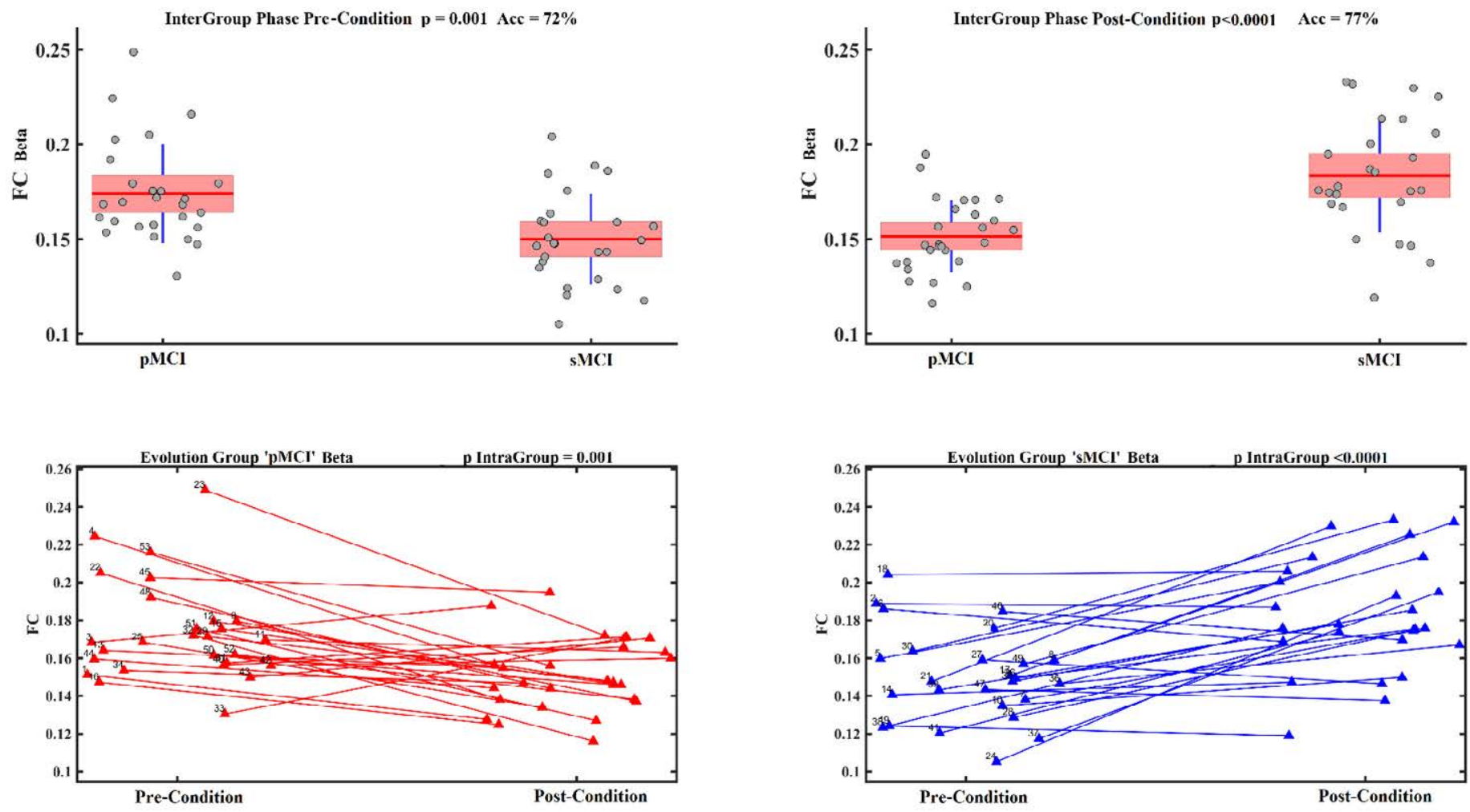

Figure 23. Evolution of each patient within each group in both conditions in beta band. 
Figure 22 shows the evolution of both groups (sMCI and pMCI) for the preto the post condition. Thus, the top-left panel shows a significant increase in synchronization in theta band for pMCI compared to sMCI in the pre-condition. Additionally, the classification analysis, using the average of the statistically significant PLV FC clusters, in the pre-condition, showed that the accuracy to discriminate between groups is about 60\% $(\mathrm{p}=0.026)$. This means that classification based on this measure only is marginally above chance. Figure 22 (top-right) also shows a significant decrease in PLV connectivity, in theta, for pMCI compared to SMCI in the post-condition. The classification analysis yielded here an accuracy of $70 \%(\mathrm{p}<0.0001)$. Finally, Figure 22 (bottom left and right) also presents the evolution of each of the subjects split by groups through pre- and post-condition. Thus, in the pMCI group $(\mathrm{p}<0.0001)$ most subjects showed decreased average PLV through conditions. However, in the sMCI group $(\mathrm{p}<0.0001)$ most of the subjects' FC average tend to increase through pre- and post-conditions.

Similarly, Figure 23 presents the evolution of both groups in the beta band through both conditions. Figure 23 (top-left) shows a significant increase in synchronization in theta band for pMCI compared to sMCI in the pre-condition. Additionally, the classification analysis, using the average of the statistically significant PLV FC clusters, in the pre-condition, showed an accuracy of $72 \%$ $(\mathrm{p}=0.001)$. Figure 23 (top-right) also shows a significant decreased in PLV connectivity, in theta, for pMCI as compared to sMCI in the post-condition. The classification analysis yielded an accuracy of 77\% ( $<<0.0001)$. Finally, Figure 23 (bottom left and right) shows the evolution of each of the subjects split by groups through pre- and post-condition. Hence, in the pMCI group $(p<0.0001)$ most of the subjects' FC average decreased through conditions. In the sMCI group $(\mathrm{p}<0.0001)$ most of the subjects' FC average tend to increase through conditions.

Importantly, when studying the PLV ratio (post/pre-condition) instead of the actual values in each of the condition, the accuracy to discriminate between groups increased to $\mathbf{9 4 . 3 4 \%}$ (sensitivity: 92.6\%, specificity: 96.15\%, PPV: 96.15\% and NPV: 92.6\%) including both clusters from theta and beta band together. 
We wanted to analyze whether these accuracies could be further increased by adding some of the other available measures. Since the correlation with the hippocampal values and the neurophysiological test showed in Figure 21, albeit significant, were rather low, it is reasonable to think that even these variables as well as the remaining neurophysiological tests, which can all be measured in a hypothetical initial study of an MCI patient, would provide additional information for the classification. Thus, we included as potential classification features the neuropsychological scores and the hippocampal volumes presented in Table 3. Indeed, we found for theta and beta band together, an accuracy of $96.23 \%$ (the highest across all the classification performed) by including the following parameters: ratio of the average FC clusters in theta and beta band, Inverse digit spam and TMTA (sensitivity: 92.6\%, specificity: 100\%, PPV: 100\% and NPV: 92.86\%).

Additionally, we studied for the classification analysis the pre- and postcondition independently using the statistically significant clusters found in theta and beta band, without adding any individual link or structural information. In the pre-condition, the average clusters in theta and beta band together with the Direct digit spam and Delayed recall classified with an accuracy of $79.25 \%$ (sensitivity: 77.8\%, specificity: 80.7\%, PPV: 80.7\% and NPV: 77.8\%).

Note, however, that these high accuracies (except the one using only the PLV in the pre- condition), present merely an academic value. In fact, it is not possible to get the PLV ratio before carrying out the second MEG scan of the longitudinal study. Yet, the significant clusters obtained can indeed be used, as shown above, in a hypothetical study in which we need to provide an estimation of the likelihood of an MCI patient to convert to AD in three years. In this case, using the average connectivity in the clusters and only two neurophysiological tests, we achieved an accuracy of $79.25 \%$, which means predicting the conversion correctly 4 out of 5 times $^{2}$.

\footnotetext{
${ }^{2}$ Of note, this accuracy did not increase when we included as features for the classifier the values of the network measures for the most significantly different nodes between groups.
} 


\section{Network Analysis}

Table 4 shows the participation coefficient averaged for statistically significant nodes in the pre-condition in beta band, including mainly brain regions localized in the temporal cortex such as both hippocampi. This network measure was higher for the pMCI than for the sMCI group.

Table 4. PLV pre-condition pMCI > sMCI in Beta Band. p-values were assessed using an ancova with age as a co-variable.

\begin{tabular}{lcc}
\hline \multicolumn{1}{c}{ Nodes } & $\begin{array}{c}\text { Participation } \\
\text { Coefficient } \\
\text { Average }\end{array}$ & p-value \\
\hline Left Rolandic operculum & 0,874364758 & 0,0235 \\
Left Hippocampus & 0,878806763 & 0,0016 \\
Right Hippocampus & 0,884515233 & 0,0263 \\
Left Parahippocampus & 0,87526415 & 0,0191 \\
Left Superior Temporal gyrus & 0,876145885 & 0,0125 \\
Left Inferior Temporal gyrus & 0,879889468 & 0,0029 \\
\hline
\end{tabular}

Additionally, the participation coefficient averaged for statistically significant nodes in the pre-condition in alpha band, included regions from the frontal, limbic and occipital cortex. In this case, the participation coefficient for these nodes was higher for sMCI than pMCI group. We assessed both p-values in Table $4 \& 5$ with an ANCOVA with age as a co-variable. 
Table 5. PLV post-condition pMCI < sMCI in Alpha Band. p-values were assessed using an ancova with age as a co-variable.

\section{Nodes}

Right Superior Frontal gyrus, Orbital

Right Middle Frontal gyrus

Right Middle Frontal gyrus, Orbital

Left Superior Frontal gyrus, Medial

Right Superior Frontal gyrus, Medial

Right Superior Frontal gyrus, Medial Orbital

Right Gyrus Rectus

Left Cingulate gyrus, Anterior part

Right Cingulate gyrus, Anterior part

Left Cingulate gyrus, Posterior part

Right Cingulate gyrus, Posterior part

Left Lingual gyrus

Left Precuneus

Right Precuneus

Right Temporal pole, Middle temporal gyrus

$\begin{array}{cc}\begin{array}{c}\text { Participation Coefficient } \\ \text { Average }\end{array} & \text { p-value } \\ 0,904463039 & 0,009 \\ 0,920828902 & 0,037 \\ 0,909295445 & 0,027 \\ 0,914451218 & 0,017 \\ 0,908558925 & 0,035 \\ 0,902233785 & 0,023 \\ 0,904974297 & 0,018 \\ 0,908988016 & 0,021 \\ 0,91098551 & 0,034 \\ 0,916840738 & 0,039 \\ 0,917111416 & 0,016 \\ 0,917162847 & 0,016 \\ 0,914155657 & 0,017 \\ 0,914615724 & 0,019 \\ 0,918509754 & 0,035\end{array}$

\section{Directed Networks based on PhTE}

When studying directed graphs by means of the PhTE, we obtained the inand out-degree for the four frequency bands studied in this chapter in the precondition for the pMCI and sMCI groups (Figure 24\&25). Qualitatively, the pMCI group exhibited a disconnected and disorganized network compared to the sMCI group. 

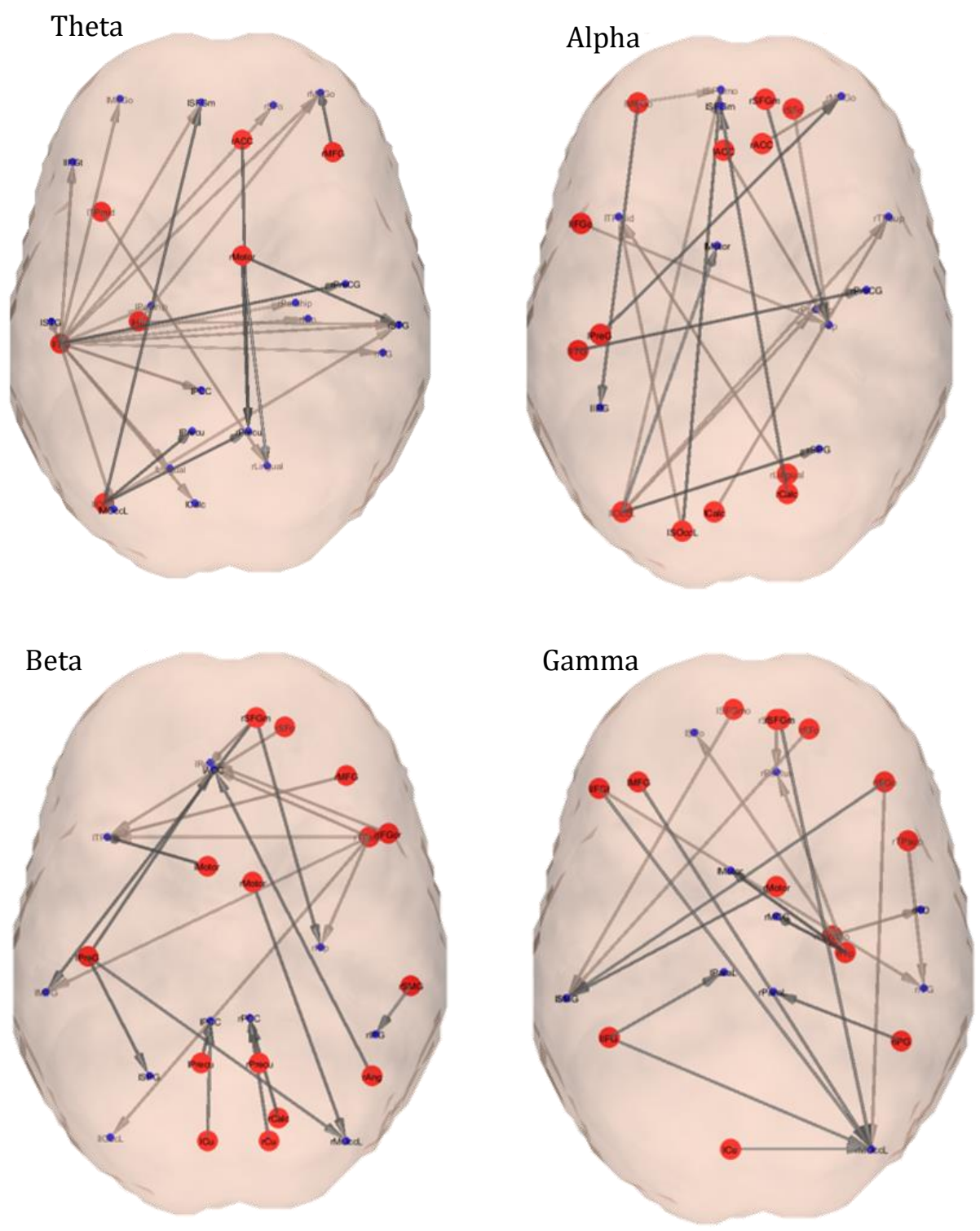

Figure 24. In- and out-degree of PhTE connectivity in the pre-condition for pMCI. In theta band, information seems to flow from temporal regions (including the hippocampus) to frontal regions. In alpha band, it can be observed a posterior to anterior information flow as well as an anterior to temporal medial regions flow pattern. Additionally, in beta band, information flows from frontal and parietal regions to the cingulate cortex. Finally, in gamma band, there is an anterior to posterior information flow pattern. 

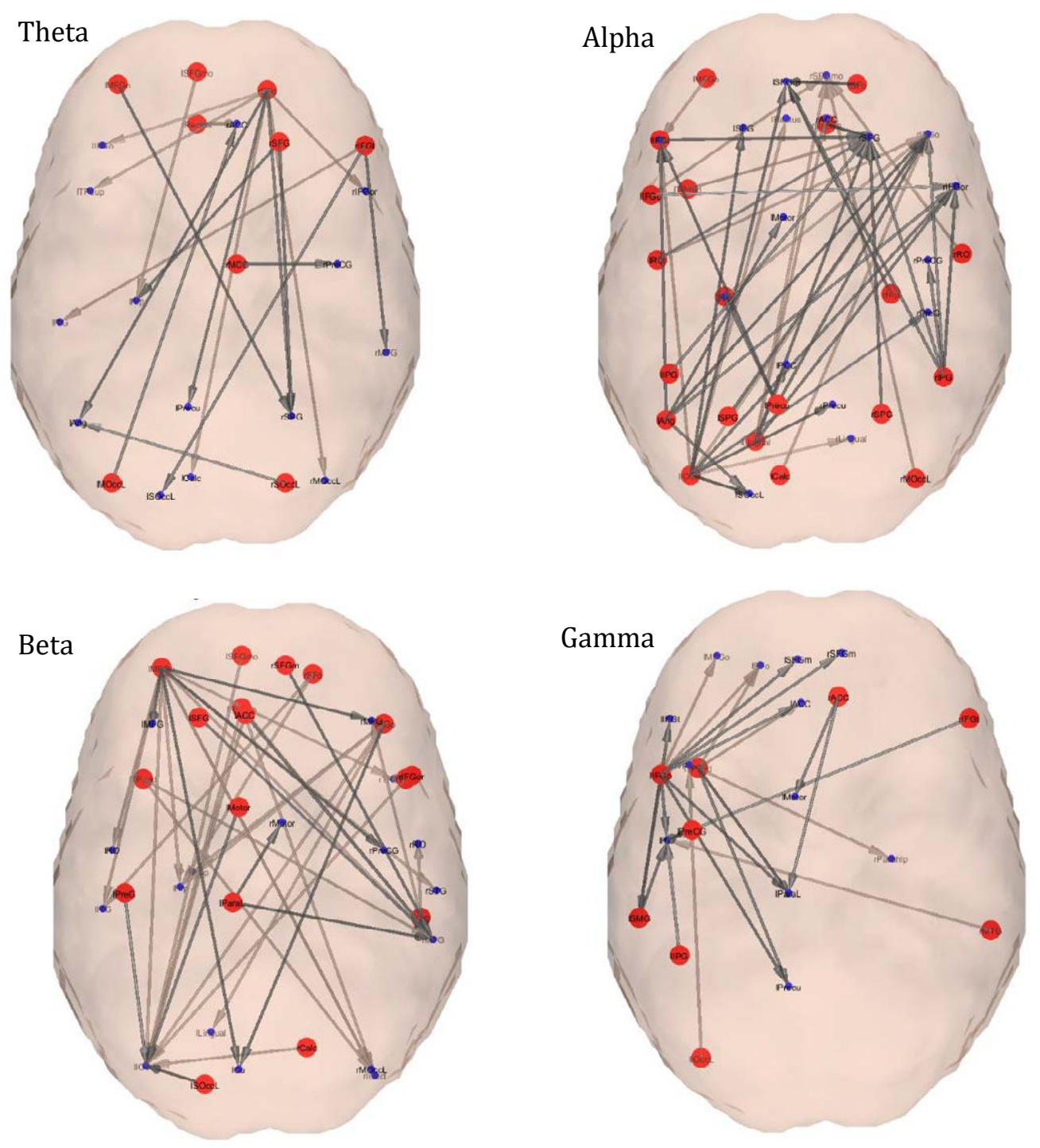

Figure 25. In- and out-degree of PhTE connectivity in the pre-condition for sMCI. In theta band, information seems to flow from frontal regions to temporal regions and frontal to occipital cortex. In alpha band, it can be observed a posterior to anterior information flow pattern. Additionally, in beta band, information flows within frontal areas as well as frontal to temporal regions including the hippocampus. Finally, in gamma band, information is flowing within the frontal cortex from posterior frontal regions to anterior ones. 


\section{Multiple Participation Coefficient (MPC)}

OSMT

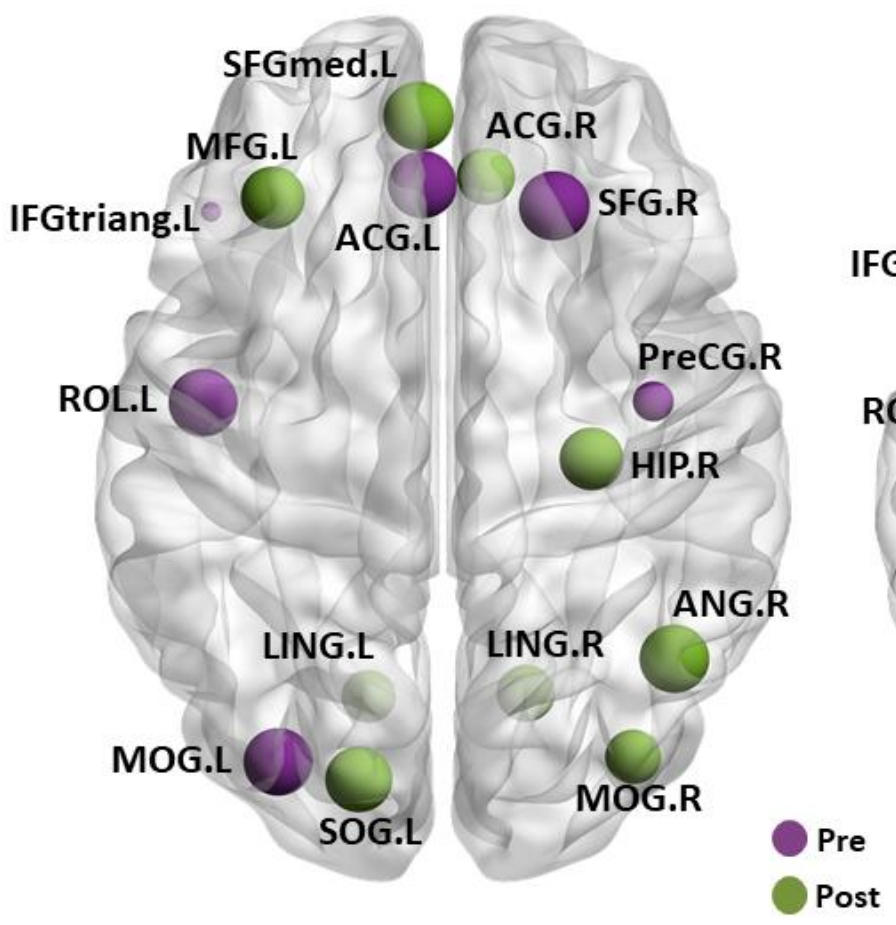

No Filter

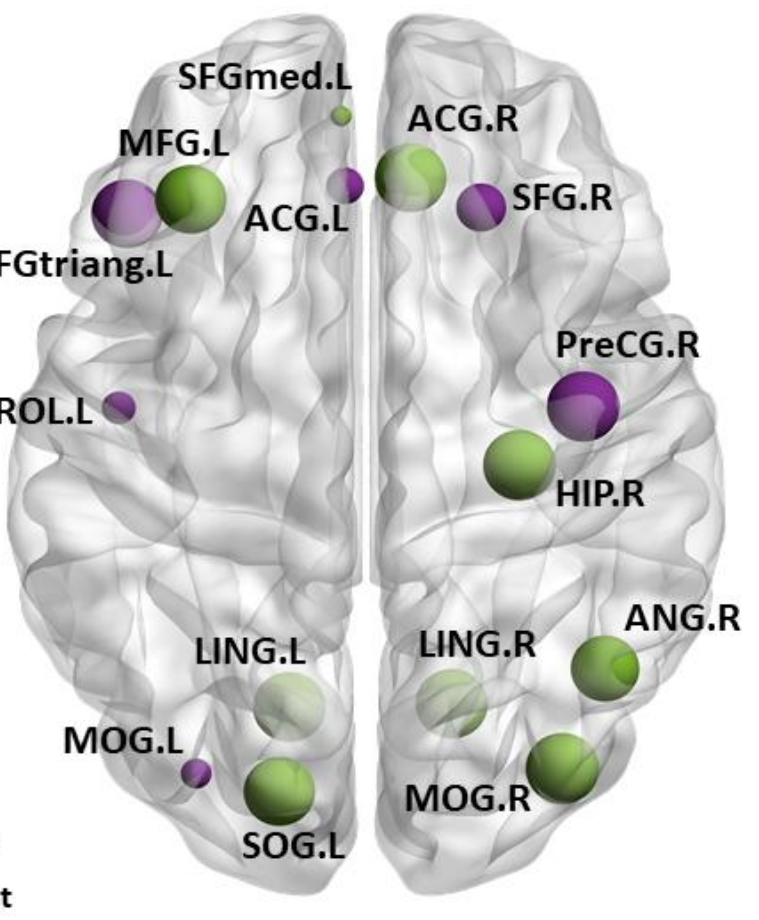

Figure 26. Multiple Participation Coefficient (MPC) for pre- (purple spheres) and post-conditions (green spheres). The size of the sphere was calculated with 1-pvalues, which means a higher and statistically significant MPC. The smaller the sphere the less or non- statistically significant node. p-values were obtained with an ancova with age as a co-variable.

In the pre-condition-> pMCI>sMCI: PreCG.R, IFGtriang.L and MOG.L. pMCI<SMCI: SFG.R, ROL.L and ACG.L

In the post-condition-> pMCI>sMCI: SFGmed.L, ACG.R, HIP.R, LING.L, LING.R, SOG.L, MOG.R and ANG.R. pMCI<sMCI: MGF.L

OMST. PLV matrix was filtered or thresholded using the OMST method to obtain the adjacency matrix.

This figure shows the nodes that actively participate in the four layers of the multilayer network.

No Filter. PLV matrix was not filtered or thresholded using any method. Instead, we used the whole weighted PLV matrix to obtain the network measures. This figure shows the nodes that actively participate in the four layers of the multilayer network. 


\section{Discussion}

Over the past years, the detection of those subjects with an increased risk of conversion to $\mathrm{AD}$ has become an important issue in clinical neuroscience. Thus, most studies have tried to demonstrate that a particular marker (i.e., demographic, cognitive, biological, etc.) was sensitive enough to predict progression to $\mathrm{AD}^{207,208}$. New perspectives aim to include combinations of markers to increase the predictive capability of the designed models 209,210 . Surprisingly, there are very few MEG studies on the progression of MCI to AD and, to our knowledge, there is no one that includes repeated measures of MEG FC progression from MCI to AD.

In this study, we observed alterations of FC patterns during the progression of $\mathrm{MCI}$ to $\mathrm{AD}$ using the PLV. Moreover, we correlated these changes with neuropsychological scores and hippocampal volumes to understand the relationship between the neural substrates and cognitive function in AD. The observed changes in FC represent evidence of altered resting state neural networks changing over time in these patients. With the resulting clusters obtained with the PLV ratio (post-/pre-condition), we defined a set of regions significant different between groups to include these clusters in a predictive model based on FC, neuropsychological scores and hippocampal volumes.

This chapter describes specific patterns of FC in resting state of those MCI patients who develop to AD. The pMCI patients showed a decrease in FC in theta and beta band as compared to sMCI. Mainly, the regions involved the frontooccipital cortex as well as fronto-temporal connections including the hippocampus.

The decrease in FC in AD seen in several studies ${ }^{34,50,56,211,212}$ can be taken as a stronger sign of network breakdown. This pattern may represent a loss of network robustness and a deviation from the optimal configuration for brain dynamic processing due to the progression of the disease 213,214 .

Although most studies have found an increase in the slow activity in AD patients, others have indicated a decrease in theta FC. These studies involved attentional and memory task-related research, demonstrating a significant reduction in theta FC in the pMCI groups as compared to sMCI212,215-217. Theta oscillations have also been implicated in working-memory activity, especially in 
medial prefrontal regions 218,219 . Medial prefrontal cortex is also a prominent target of $\mathrm{AD}$, however, mostly in later stages 220,221 .

It is noteworthy that this desynchronization in theta band is related to memory task-related activity as mentioned above. In fact, several studies have found strong correlations between neuropsychological variables (especially those involved in memory functioning) and $\mathrm{FC}^{50,221,222}$. Decreased FC in the theta band, in particular between frontal regions, is becoming increasingly associated with working memory processes $223-225$.

Furthermore, this disruption in FC can also be explained by connectional diaschisis ${ }^{164,226}$. This phenomenon describes the structural/functional association between regions with high amyloid accumulation and the hippocampus that might affect the ability of this area to share information with other brain regions, resulting in connectivity reduction and consequently in a reduced neuronal input from those regions.

In our case, we found positive correlations between the immediate and delayed recall with the average ratio of the FC cluster in theta band. This relationship suggests that the variation of the PLV FC through time mirrors the increase in memory impairment. This network disruption may reflect a pathological activity that exerts a negative impact on memory function, and may increase the risk of conversion to $\mathrm{AD}^{46}$.

Moreover, the occipital lobe is usually been considered impaired until the last stages of the $\mathrm{AD}^{227-229}$. Thus, our results suggest that the occipital lobe appears to be part of a disrupted network in which pMCI patients present a decreased synchronization compared to sMCI subjects.

Additionally, we found a decreased beta band synchronization. Beta band may have a special significance in $\mathrm{AD}$, especially in the early stages of the disease $\mathrm{e}^{230}$.

It is classically related to excitatory activity and cognitive processes that deteriorate during $\mathrm{AD}^{142}$. Our results are similar to those of Koenig et al ${ }^{142}$, who found that beta band synchronization was lower in AD patients than in patients 
with MCI. Also, Koelewijn et $\mathrm{al}^{231}$ found a decreased in functional connectivity in beta band in resting state MEG in AD patients.

Desynchronization in certain brain regions such as the hippocampus is related with brain atrophy that is characteristic of AD. Normally, theta rhythms characterize hippocampal/parahippocampal-cortical interactions for focused attention, working memory, and encoding processes ${ }^{88}$. In the case of AD patients, the hippocampus is usually atrophied and its functionality is impaired.

Thus, we observed a positive correlation between the average ratio of $\mathrm{FC}$ in theta and beta band between the right and left hippocampus volumes. This variation of FC appears as a desynchronization in both bands in $\mathrm{pMCI}$, suggesting that this may be related with the decreased in hippocampal volume. This supports the notion that the disruption of anatomical networks influences brain organization at the functional level, resulting in the manifestations of $\mathrm{MCI}^{58}$. In addition, it is well known that brain atrophy in $\mathrm{AD}$ is characteristic especially in the hippocampus. Several studies have reported a relationship between hippocampal volumes and functional connectivity in $\mathrm{AD}^{46,50,143}$.

\section{Evolution of brain synchronization through pre- and post-condition in pMCI and SMCI}

The changes in FC showed here represent evidence of network disruption associated to the progression of $\mathrm{AD}$, as shown in Figure 22\&23. In addition, it can be conceived that during the course of the disease, FC fluctuates, with a possible initial increase and a later decrease ${ }^{143}$. Notably, pMCI presents a higher connectivity compared to the sMCI in the pre-condition, possibly indicating MCI characteristic hyper-synchronization due to a compensatory mechanism to supply the cognitive impairment as well as the synaptic loss $137,232,233$. Then, in the post condition, pMCI patients showed a significant decrease in connectivity compared to the sMCI. As mentioned above, this specific desynchronization is characteristic of the AD pathology. In addition, the sMCI behave like the pMCI group but delayed in time, that is, in the pre-condition they showed less connectivity than the pMCI because their state is probably not as advanced as the pMCI and in the post- 
condition it can be seen how connectivity increases in sMCI probably showing the characteristic connectivity patterns of MCI patients.

\section{Predictive Models to distinguish pMCI from sMCI}

For an integrative approach of the impact of FC, neuropsychological scores and hippocampal volumes, a classification analysis was performed, with these variables acting as predictors to create a model that allows the classification of pMCI and sMCI. Several studies have focused on the conversion from MCI to AD from different perspectives, and the findings suggest the involvement of different frequency bands as predictors of conversion ${ }^{234-236}$. Nevertheless, in the literature, these studies focused in one or two variables as predictor and they do not present MEG neural substrates across time. The idea of a longitudinal study to describe the specific patterns of the disease during its progression helps us to define clusters that included in a prediction model allow us the classification between sMCI and pMCI.

Despite promising developments in imaging and CSF biomarkers, there are limitations to their widespread use because not all the time these techniques are available in all the studies. An advantage of this approach is that we could use a predictive model based on easy and non-invasive access data such as the MEG and neuropsychological scores.

As commented above, it is important to consider that the model designed here with the average FC ratio does not have clinical value, as it not possible to have the post-MEG measurement at the beginning of the patient's diagnosis. This model has, therefore, merely an academic value to define and study the progression of the disease. The predictive model includes both theta and beta band average ratio of the FC clusters and two neuropsychological scores: TMTA and inverse digit spam often related to working memory, attentional and executive functions. As mentioned before, the alteration in theta band are related with working memory impairment in AD. Additionally, AD patients present a decline in attentional and executive functions through the progression of the disease $32,137,237$. Due to the impairment of these cognitive functions in $\mathrm{AD}$, it makes sense that these variables support the classification of these patients. 
In addition, to provide a model as close as possible to the clinical approach, we trained a predictive model based on the pre-condition FC and the most informative neurophysiological tests (as measured by the accuracy of the model itself). Thus, we found an accuracy of $79.25 \%$ to discriminate between pMCI and sMCI with the average ratio of the clusters in theta and beta found in the connectivity analysis, the direct digit spam and delayed recall. These findings suggest that the network disruption and the neuropsychological scores related with memory are good predictors between groups. However, in a previous study performed with this data, Lopez et $\mathrm{al}^{46}$ found a model based on a FC link and the immediate recall with higher accuracy. For future studies, the inclusion of neuropsychological assessment and hippocampal volumes in the post-condition can increase the accuracy of the predictive model.

\section{Network alterations observed with graph analysis}

With the aim to characterize and describe the network organization of both groups we performed an additional network analysis based on the participation coefficient for the PLV and in- and out-degree for PhTE in resting state MEG data in the pre-condition.

We identified nodes that participate in many sub-networks of the brain. These nodes are candidate for brain hubs. Hubs exist in many real-world networks and they often play critical roles in facilitating communication and maintaining network integrity 238,239 .

We found these candidate hubs using the participation coefficient with PLV matrices. Hubs with low participation coefficients are called "provincial" hubs because their links are not distributed widely among communities, whereas hubs with higher participation coefficients are called "connector" hubs ${ }^{193}$.

In Table 4, the temporal lobe, specifically the hippocampus, presents a higher participation coefficient in beta band in the pre-condition for the pMCI than sMCI and can be seen as a "connector" hub. As mentioned earlier, these regions are involved in memory functioning and in AD are related with memory impairment. In the connectivity results, we mentioned that there was a specific desynchronization in theta band in pMCI, in certain areas such as the hippocampus. This disruption and the high participation coefficient found here 
may indicate that due to the impairment the hippocampus breaks its local connections, has to find a way to compensate the deficit and connect with other brain areas to support its functionality. This can be thought as an aberrant functioning 34,191 .

In Table 5, it can be appreciated that all the nodes have a low participation coefficient in pMCI compared to sMCI group in alpha band. Intriguingly, most of these nodes belong to the well-known DMN, which is usually disrupted in AD patients $^{240}$. The low participation here may indicate that these nodes are not participating in other sub-networks probably due to the common functional desynchronization in AD. On the contrary, the sMCI presents high participation coefficient in these brain regions which possibly means that this network is better communicated with other sub-networks in the brain.

Although the PhTE analysis is an exploratory and qualitative one, from Figure 24\&25, we can see already the disorganization and disruption of the PhTE networks of the pMCI patients compared to the sMCI in all the frequency bands. A recent study ${ }^{241}$ with PhTE has found that the disruption of information flow may underlie clinical symptoms in AD. Mainly, they found it to be disturbed in the beta band. They concluded that AD pathology might affect the flow of information between brain regions.

Specifically, in Figure 24, in theta band we found that information seems to flow from temporal regions (including the hippocampus) to frontal regions. This would give us an idea of the directionality of the network in this specific frequency band with respect to our findings in the FC analysis. In addition, in alpha band can be seen a posterior to anterior pattern of information flow, this has been reported in a recent study about the directionality of the network in AD patients ${ }^{241}$. Moreover, Juan-Cruz et $\mathrm{al}^{242}$ found a disrupted EC pattern for theta, beta and gamma bands. In theta band, specific patterns from left-temporal to anterior region support our findings. Similarly, disconnection in theta and beta band was reported using $\mathrm{DTF}^{243}$. In the case of beta band, they found a decrease in the information flow from posterior to anterior regions. Results in gamma band indicate the presence of connectivity decrements in the direction of posterior region, which agrees with previous MEG studies ${ }^{244}$. 
Finally, in order to extend the characterization of the network, we studied the MPC in two ways: analyzing the whole weighted PLV matrix and thresholding the PLV matrix with the OMST. As mentioned before, the MPC is the multi-layer version of the participation coefficient. This index quantifies the participation of each node in the four MEG frequency layers. Thus, nodes with high MPC are considered central hubs as they would allow for a better information exchange among different layers ${ }^{196}$. This approach may contain important information that cannot be revealed by frequency-specific brain networks analysis.

First, we found that both methods were not consistent statistically. In the pre- and post-condition, the significant results varied according to the method used. Hence, in the pre-condition, high MPC can be seen in frontal regions for the pMCI compared to the sMCI as well as in the anterior cingulate gyrus for the sMCI compared to the pMCI. In addition, in the post condition we found that the 'No filter' method (i.e., the whole matrix) present more significant nodes in frontal regions, anterior cingulate, hippocampus and occipital lobes for the pMCI compared to the sMCI.

The high participation of frontal regions in the pre-condition with other layers in the brain may be due to a compensatory mechanism because of the cognitive deficits at disease onset 237,245 . Likewise, in the post-condition, the fact that several nodes are in communication with other layers may be due to the network failure ${ }^{34,246}$. Thus, these regions are trying to supply their functionalities through other paths and layers. As mentioned above, this dysfunction is very characteristic of $\mathrm{AD}^{247}$. Additionally, some of these regions such as the cingulate cortex and hippocampus are highly involved in AD and the DMN. Usually during the course of the disease, these areas are damaged, and the communication to other areas impaired 247,248 .

In sum, in this work we have studied the progression of AD through different perspectives. These results suggest that MEG FC is able to detect the functional alterations caused by synaptic disruption. In addition, EC supported the network disruption hypothesis in $\mathrm{AD}$ from the qualitative analysis presented here. Thus, this study shows that MEG is a promising biomarker of synaptic disruption in the prediction of $\mathrm{AD}$, and the importance of combining it with other measures 
such as neuropsychological scores $46,210,235$ to develop a predictive model of AD. Future studies should focus on predicting future cognitive decline in the preclinical stages of the disease ${ }^{12,208}$ to initiate preventive treatments at the earliest possible time.

Brain connectivity allows us a better understanding of brain functioning and how the disease causes network disruption and consequently affects the cognitive status of the patients ${ }^{249}$. Therefore, the present work provides a new perspective to study the evolution of $A D$ and supports the MEG as an important biomarker to evaluate $\mathrm{AD}$ progression. 


\section{Chapter 6}

\section{Conclusions}

In this thesis, we studied the progression from MCI to AD through $\mathrm{FC}$ and MEG to reinforce MEG and FC as important tools to detect early synaptic dysfunction associated with AD brain pathology.

In the next paragraphs, we will summarize the main findings of each chapter, and how each study helps us to understand AD from a FC perspective.

\section{Main findings}

In chapter 3, we studied source space PLV FC patterns of healthy elderly subjects under scopolamine and placebo administration. The aim of this work was to test if scopolamine administration will produce similar changes in brain connectivity as those observed in $\mathrm{AD}$, thereby verifying a possible model of $\mathrm{AD}$ impairment. Thus, we found a general decrease in synchronization in most of the regions in most of the frequency bands analyzed. In particular, in the delta band the connectivity between left parahippocampal and right occipital interior cortex increases under the scopolamine. On the contrary, in alpha, beta and gamma bands the PLV decreases between different brain regions under scopolamine.

Additionally, we described the architecture of the functional brain network of the subjects by means of two indices: the clustering coefficient and the average shortest path length. As a result, clustering significantly decreased while shortest path length significantly increased in alpha band both after scopolamine administration.

Whereby, our findings indicate that PLV and graph analysis are suitable tools to measure brain connectivity changes induced by scopolamine, which causes alterations in brain connectivity apparently similar to those reported in AD. 
In chapter 4, we used MEG to obtain PLV FC patterns associated with abnormal CSF levels of p-tau and $A \beta-42$ in patients with MCI. We also aimed to determine an association between these patterns and structural connectivity alterations and the convergent impact of neuropsychological scores and CSF variables on network disorganization.

Patients with abnormal CSF p-tau and A $\beta-42$ levels exhibited both reduced and increased functional synchronization affecting several brain regions such as the anterior/posterior cingulate cortex, orbitofrontal cortex, and medial temporal areas in different frequency bands. Additionally, a reduction in posterior cingulate functional connectivity mediated by p-tau was associated with impaired axonal integrity of the hippocampal cingulum.

Several connectivity alterations were predicted by CSF biomarkers and cognitive scores. Thus, CSF levels of the biomarkers predicted most of the aberrant functional connections seen in these MCI patients. In particular, a decrease in synchronization of the medial temporal cortex was associated with $A \beta-42$. This region is thought to have a low $A \beta$ burden, particularly in early stages of the disease. In addition, the free recall score showed a predictive value for intrahemispheric anterior cingulate-medial temporal connectivity, whereas the MMSE score predicted functional connectivity between the inferior temporal and precentral cortex.

In sum, this study indicated that CSF abnormal levels of p-tau and $A \beta-42$ in early $\mathrm{AD}$ are related with network disruption involving desynchronization and hypersynchronization of key regions of the default mode network and temporal areas.

In chapter 5, we studied PLV FC and PhTE EC patterns associated to neuropsychological scores and structural alterations to study the progression from MCI to AD, specifically, in MCI patients who underwent a 3 years MEG follow-up study. The aim of this work was to characterize connectivity changes of pMCI and sMCI and include these patterns together with neuropsychological and structural variables in a predictive model.

Thus, theta and beta band in PMCI compared to SMCI exhibited PLV ratio desynchronization. In addition, network alterations correlated with immediate, 
delayed recall and the hippocampal volumes, these associations suggested that the variation of the PLV FC through time is reflected in memory impairment.

When studying both conditions independently, pMCI presented a higher connectivity compared to the sMCI in the pre-condition. Then, in the post condition, pMCI patients showed a significant decrease in connectivity compared to the SMCI.

Additionally, a predictive model with an accuracy of $96.23 \%$ including both theta and beta band average ratio of the FC clusters and two neuropsychological scores: TMTA and inverse digit spam was proposed. Furthermore, a predictive model was proposed based on the pre-condition FC to discriminate between pMCI and sMCI with the average ratio of the clusters in theta and beta, the direct digit spam and delayed recall reaching an accuracy of $79.25 \%$.

The qualitative graph analysis led us to find in the functional networks studied with PLV, a high participation coefficient in temporal regions such as the hippocampus in pMCI compared to sMCI in the pre-condition in beta band. Interestingly, regions involved in the DMN exhibited low participation coefficient, in pMCI than sMCI, in the post-condition in alpha band. Finally, pMCI showed a EC disorganized and disrupted network in all the frequency bands analyzed compared to $\mathrm{sMCI}$.

To complement these findings, pMCI in the frontal regions exhibited high MPC compared to the sMCI. On the contrary, in the anterior cingulate gyrus for the sMCI compared to the pMCI. In the post condition, 'No filter' method included more significant nodes in frontal regions, anterior cingulate, hippocampus and occipital lobes for the pMCI compared to the sMCI. The involvement of these regions with other layers in the MEG multiplex network may indicate a mechanism to compensate the network disruption and the cognitive deficits present in AD.

Overall, this thesis supports that resting state MEG FC is a key technique to study brain pathologies such as $\mathrm{AD}$ and to measure changes in functional networks that may reflect abnormalities due to the development of a neuropathological processes. Additionally, the study of the brain in a resting state condition provided a reliable measure, which allowed us to characterize the brain activity over time, 
probably made more accurate comparisons between patients, and potentially employable in a clinical approach.

\section{Limitations and methodological considerations}

One important limitation of the studies presented in this thesis is the sample size. Drug administration and CSF extraction are techniques that do not allow a widespread use and thus it was not possible to obtain greater samples. Unfortunately, from small samples, important conclusions cannot be made whence an adequate statistical analysis should be taken in consideration.

Another limitation of this thesis is that all the results obtained are based on a MEG processing pipeline that includes the choice of a variety of available methods such as the pre-processing, filtering, source reconstruction, the computation of FC and EC and graph analysis. Until today, there is no agreement as to the ideal processing steps to perform FC analysis due to each protocol and the choice of the methods will depend on the hypothesis of the study.

Finally, different authors have questioned the use of PLV due to its sensitivity to zero-lag (and thereby to source leakage effects) in FC studies ${ }^{250-254}$. This measure estimates the extent of FC using the instantaneous phases of the signals under the hypothesis that connected brain regions will generate signals whose instantaneous phases evolve together. In this case, the phases of the signals are said to be "locked" and the phase difference must be constant (PLV=1). However, real-world signals are inherently noisy, and it is not always possible to be sure that the evaluated signal only comes from one source 255 .

Essentially, PLV does not discriminate between zero and non-zero-lag interactions. Therefore, this measure cannot distinguish directed real connectivity (non-zero-lag) from undirected connectivity or source leakage, normally occurring at zero-lag. Thus, when selecting PLV as a connectivity measure we have to take into account two limitations or special characteristics of this method:

1) When reconstructing MEG time series in source-space, the number of sources is much larger than the number of MEG sensors. Then, the lack of spatial resolution makes that the activity of one sensor appears in 
several sources at the same time, when they are in the vicinity, a phenomenon known as source leakage. In this case, the ROIs would have a non-zero PLV if they are close to each other because of the low spatial resolution of the technique compared to the size of the sources.

2) Since PLV includes the zero-lag synchronization and this effect has always been considered non-real because it is believed that the interaction between two highly synchronized distant regions may be influenced by a third source in between causing non-true interactions.

In this context, several connectivity measures have been proposed to discard zero-lag synchronization such as PLI ${ }^{250}$, PhTE ${ }^{185}$ on the phases, and orthogonalized Amplitude Envelope Correlation ${ }^{153,231}$ on the amplitude, among others. The main limitations of these methods are that they remove information that may be relevant, i.e., true zero-lag FC, and that they lack reliability 251,256 . Indeed, several studies have demonstrated that zero-lag connectivity may be real and not caused by spurious connectivity. Specifically, in cortico-thalamo-cortical interactions, in which two cortical brain areas synchronized through an intermediate brain region, in this case the thalamus. These interactions are known as dynamic relaying, which contributes to large-scale cortical communication. These studies present experimental and theoretical evidence that the conduction delays only depend on the difference between the distance, in time, the intermediate element (known as relay element) and the two interconnected brain regions ${ }^{257-259}$.

In this way, when choosing a FC method, one has to face a decision based on selecting a metric that discards information or one that includes all the information. It is intrinsically difficult to discriminate between two things that in the mirror look the same. Thus, advantages of PLV includes high reliability251,256 and reproducibility. High reliability ensures less variability within groups; therefore, for diagnostic purposes it should be potentially better. 


\section{Future directions}

We intend, in future works, to exploit the longitudinal study presented in chapter 5 of this thesis. First, we plan to extend the study of EC with PhTE with the two MEG recordings and make comparisons between group and conditions with an adequate statistical analysis to test for changes in FC as well as the possible association with the neuropsychological and hippocampal volumes. Additionally, we should address the predictive value of these alterations.

Furthermore, future studies should focus on network properties such as the participation coefficient or other metrics that help in the network characterization of the FC/EC patterns found.

The study of multiplex MEG networks could add more information in the study of connectivity and graph theory, studying the interactions of all the frequency bands analyzed ${ }^{260}$. This is a relatively new field that has much to be explored and that can give interesting evidence regarding the interactions between brain regions and each layer in this case corresponding to the frequency bands. Specially, to identify potential hubs in the study of AD.

Additionally, it would be interesting to study the association between CSF, neuropsychological and structural variables with the longitudinal study that includes two MEG measurements and thus, to link up the pathological accumulation of $A \beta$ and tau proteins with cognitive and memory impairment and hippocampal atrophy with connectivity pattern alterations over time. 


\section{Appendix A}

\begin{tabular}{|c|c|c|c|c|c|}
\hline Region & Abbreviations & Cortical and sub-cortical Regions & Region & Abbreviations & Cortical and sub-cortical Regions \\
\hline 1 & REC.L & Left Gyrus Rectus & 46 & SFGdor.R & Right Superior frontal gyrus, dorsolateral \\
\hline 2 & OLF.L & Left Olfactory Cortex & 47 & MFG.R & Right Middle frontal gyrus \\
\hline 3 & ORBsup.L & Left Superior frontal gyrus, orbital part & 48 & IFGoperc.R & Right Inferior frontal gyrus, opercular part \\
\hline 4 & ORBsupmed.L & $\begin{array}{l}\text { Left Superior frontal gyrus, medial } \\
\text { orbital }\end{array}$ & 49 & IFGtriang.R & Right Inferior frontal gyrus, triangular part \\
\hline 5 & ORBmid.L & Left Middle frontal gyrus orbital part & 50 & SFGmed.R & Right Superior frontal gyrus, medial \\
\hline 6 & ORBinf.L & Left Inferior frontal gyrus, orbital part & 51 & SMA.R & Right Supplementary motor area \\
\hline 7 & SFGdor.L & Left Superior frontal gyrus, dorsolateral & 52 & PCL.R & Right Paracentral lobule \\
\hline 8 & MFG.L & Left Middle frontal gyrus & 53 & PreCG.R & Right Precentral gyrus \\
\hline 9 & IFGoperc.L & Left Inferior frontal gyrus, opercular part & 54 & ROL.R & Right Rolandic operculum \\
\hline 10 & IFGtriang.L & $\begin{array}{l}\text { Left Inferior frontal gyrus, triangular } \\
\text { part }\end{array}$ & 55 & PoCG.R & Right Postcentral gyrus \\
\hline 11 & SFGmed.L & Left Superior frontal gyrus, medial & 56 & SPG.R & Right Superior parietal gyrus \\
\hline 12 & SMA.L & Left Supplementary motor area & 57 & IPL.R & $\begin{array}{l}\text { Right Inferior parietal, but supramarginal and } \\
\text { angular gyri }\end{array}$ \\
\hline 13 & PCL.L & Left Paracentral lobule & 58 & SMG.R & Right Supramarginal gyrus \\
\hline 14 & PreCG.L & Left Precentral gyrus & 59 & ANG.R & Right Angular gyrus \\
\hline 15 & ROL.L & Left Rolandic operculum & 60 & PCUN.R & Right Precuneus \\
\hline 16 & PoCG.L & Left Postcentral gyrus & 61 & SOG.R & Right Superior occipital gyrus \\
\hline 17 & SPG.L & Left Superior parietal gyrus & 62 & MOG.R & Right Middle occipital gyrus \\
\hline 18 & IPL.L & $\begin{array}{l}\text { Left Inferior parietal, but } \\
\text { supramarginal and angular gyri }\end{array}$ & 63 & IOG.R & Right Inferior occipital gyrus \\
\hline 19 & SMG.L & Left Supramarginal gyrus & 64 & CAL.R & Right Calcarine fissure and surrounding cortex \\
\hline 20 & ANG.L & Left Angular gyrus & 65 & CUN.R & Right Cuneus \\
\hline 21 & PCUN.L & Left Precuneus & 66 & LING.R & Right Lingual gyrus \\
\hline 22 & SOG.L & Left Superior occipital gyrus & 67 & FFG.R & Right Fusiform gyrus \\
\hline 23 & MOG.L & Left Middle occipital gyrus & 68 & HES.R & Right Heschl gyrus \\
\hline 24 & IOG.L & Left Inferior occipital gyrus & 69 & STG.R & Right Superior temporal gyrus \\
\hline 25 & CAL.L & $\begin{array}{l}\text { Left Calcarine fissure and surrounding } \\
\text { cortex }\end{array}$ & 70 & MTG.R & Right Middle temporal gyrus \\
\hline 26 & CUN.L & Left Cuneus & 71 & ITG.R & Right Inferior temporal gyrus \\
\hline 27 & LING.L & Left Lingual gyrus & 72 & TPOsup.R & Right Temporal pole: superior temporal gyrus \\
\hline 28 & FFG.L & Left Fusiform gyrus & 73 & TPOmid.R & Right Temporal pole: middle temporal gyrus \\
\hline 29 & HES.L & Left Heschl gyrus & 74 & PHG.R & Right Parahippocampal gyrus \\
\hline 30 & STG.L & Left Superior temporal gyrus & 75 & ACG.R & Right Anterior cingulate and paracingulate gyri \\
\hline 31 & MTG.L & Left Middle temporal gyrus & 76 & DCG.R & Right Median cingulate and paracingulate gyri \\
\hline 32 & ITG.L & Left Inferior temporal gyrus & 77 & PCG.R & Right Posterior cingulate gyrus \\
\hline 33 & TPOsup.L & $\begin{array}{l}\text { Left Temporal pole: superior temporal } \\
\text { gyrus }\end{array}$ & 78 & INS.R & Right Insula \\
\hline 34 & TPOmid.L & $\begin{array}{l}\text { Left Temporal pole: middle temporal } \\
\text { gyrus }\end{array}$ & 79 & HIP.L & Left Hippocampus \\
\hline 35 & PHG.L & Left Parahippocampal gyrus & 80 & HIP.R & Right Hippocampus \\
\hline 36 & ACG.L & $\begin{array}{l}\text { Left Anterior cingulate and paracingulate } \\
\text { gyri }\end{array}$ & 81 & AMYG.L & Left Amygdala \\
\hline 37 & DCG.L & $\begin{array}{l}\text { Left Median cingulate and paracingulate } \\
\text { gyri }\end{array}$ & 82 & AMYG.R & Right Amygdala \\
\hline 38 & PCG.L & Left Posterior cingulate gyrus & 83 & CAU.L & Left Caudate \\
\hline 39 & INS.L & Left Insula & 84 & CAU.R & Right Caudate \\
\hline 40 & REC.R & Right Gyrus Rectus & 85 & PUT.L & Left Putamen \\
\hline 41 & OLF.R & Right Olfactory Cortex & 86 & PUT.R & Right Putamen \\
\hline 42 & ORBsup.R & Right Superior frontal gyrus, orbital part & 87 & PAL.L & Left Pallidum \\
\hline 43 & ORBsupmed.R & $\begin{array}{l}\text { Right Superior frontal gyrus, medial } \\
\text { orbital }\end{array}$ & 88 & PAL.R & Right Pallidum \\
\hline 44 & ORBmid.R & Right Middle frontal gyrus orbital part & 89 & THA.L & Left Thalamus \\
\hline 45 & ORBinf.R & Right Inferior frontal gyrus, orbital part & 90 & THA.R & Right Thalamus \\
\hline
\end{tabular}

Table 6. Abbreviations for the 90 regions of the AAL atlas used in the study presented in chapter 5 . For all the analysis the subcortical regions were removed. 


\section{Chapter 5}

\section{Network Analysis - PhTE}

When computing the PhTE, the interaction lag is an important parameter to take into account. The network analyses involve multiple nodes connected via neurons of different degrees of myelination and different lengths, resulting in different interaction lags across node pairs. In addition, the values for these interaction lags are unknown quantities ${ }^{185}$. This interaction lag between nodes is modeled in PhTE as an a priori chosen analysis lag.

Lobier et al ${ }^{185}$ proposed the PhTE method and in their work they demonstrated that the choice of the delay does not influence the results. They chose to use local maxima of a time-lagged synchronization index (Phase TD-MI) to determine an optimal analysis lag.

Additionally, Hillebrand et $\mathrm{al}^{187}$ and Engels et $\mathrm{al}^{241}$ computed the PhTE prediction delay as $(\mathrm{Ns} \times \mathrm{Nch}) / \mathrm{N} \pm$, with $\mathrm{Ns}$ and $\mathrm{Nch}$ the number of samples and channels (ROIs), respectively, and $\mathrm{N} \pm$ the number of times the phase changes sign across time and channels. Although they do not argue the rationale behind it.

For this analysis, we chose the period corresponding to the mean frequency within each band, as we found it is the value giving rise to a maximum of PhTE and to plot it we chose $10,20,30 \%$ above and under the behavior of the function depending on the values of the lags.

Figure 27 shows the PhTE computed with different values for the interaction lag, supporting the idea that in the period of the mean frequency, the PhTE reaches its maximum value. 

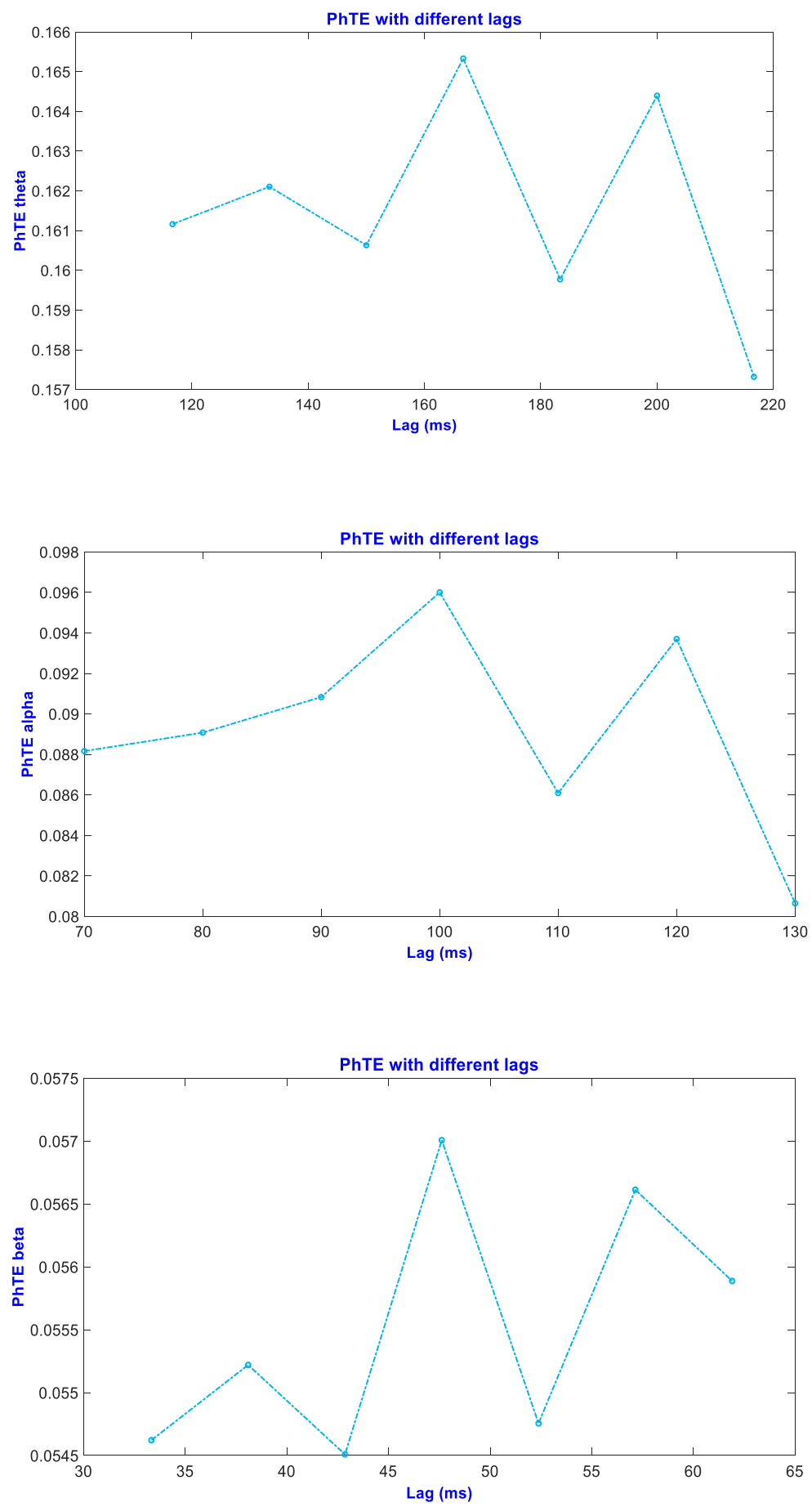

Figure 27.Phte for a wide range of analysis lags for different frequency bands 


\section{References}

1. Alzheimer's Association. 2017 Alzheimer's Disease: Facts and Figures.

2. Policy Brief: The Global Impact of Dementia 2013-2050; Policy Brief: The Global Impact of Dementia 2013-2050.

3. World Alzheimer Report 2015 The Global Impact of Dementia AN ANALYSIS OF PREVALENCE, INCIDENCE, COST AND TRENDS.

4. Aulestia Urrutia, K. El Alzheimer En España, Situación actual de la enfermedad y su investigación. (2013).

5. Report, T. Estudio sobre las enfermedades neurodegenerativas en España y su impacto económico y social. (2016). doi:10.13140/RG.2.1.3269.8009

6. Alzheimer, A. Uber eine eigenartige Erkrankung der Hirnrinde. Allg Zeitschr Psychiatr 64, 146-148 (1907).

7. Ryan, N. S., Rossor, M. N. \& Fox, N. C. Alzheimer's disease in the 100 years since Alzheimer's death. doi:10.1093/brain/awv316

8. Scahill, R. I., Schott, J. M., Stevens, J. M., Rossor, M. N. \& Fox, N. C. Mapping the evolution of regional atrophy in Alzheimer's disease : Unbiased analysis of fluid-registered serial MRI. Proc Natl Acad Sci US A 99, 1-5 (2002).

9. Lau, L.-F., Brodney, M. A. \& Berg, S. (Stefan). Alzheimer's disease. (Springer, 2008).

10. Brill, M. T. Alzheimer's disease. (Benchmark Books, 2005).

11. Welsh, E. M. Frontiers in Alzheimer's disease research. (Nova Science Publishers, 2006).

12. López-Sanz, D. et al. Functional connectivity disruption in subjective cognitive decline and mild cognitive impairment: A common pattern of alterations. Front. Aging Neurosci. 9, 1-12 (2017).

13. Vos, S. J. B. et al. Preclinical Alzheimer's disease and it's outcome: a longitudinal cohort study. Lancet Neurol 12, 957-965 (2014).

14. Sperling, R. A. et al. Toward defining the preclinical stages of Alzheimer's disease: Recommendations from the National Institute on Aging-Alzheimer's Association workgroups on diagnostic guidelines for Alzheimer's disease. Alzheimer's Dement. 7, 280292 (2011).

15. Lazarczyk, M. J., Hof, P. R., Bouras, C. \& Giannakopoulos, P. Preclinical Alzheimer disease: identification of cases at risk among cognitively intact older individuals. BMC Med. 10, 127 (2012).

16. Albert, M. S., Moss, M. B., Tanzi, R. \& Jones, K. Preclinical prediction of AD using neuropsychological tests. J. Int. Neuropsychol. Soc. 7, 631-639 (2001).

17. Chong, M. S., Sahadevan, S., Tan, J. \& Seng, T. Preclinical Alzheimer 's disease : diagnosis and prediction. 4, (2005).

18. Samtani, M. N. et al. Disease progression model in subjects with mild cognitive impairment from the Alzheimer's disease neuroimaging initiative: CSF biomarkers predict population subtypes. Br. J. Clin. Pharmacol. 75, 146-61 (2013).

19. Jack, C. R. \& Holtzman, D. M. Biomarker modeling of alzheimer's disease. Neuron 80, 13471358 (2013).

20. Bird, T. D. Alzheimer Disease Overview. GeneReviews(®) (University of Washington, Seattle, 1993).

21. Michaelson, D. M. APOE $\varepsilon 4$ : The most prevalent yet understudied risk factor for Alzheimer's disease. Alzheimer's Dement. 10, 861-868 (2014). 
22. Liu, C.-C., Kanekiyo, T., Xu, H., Bu, G. \& Bu, G. Apolipoprotein E and Alzheimer disease: risk, mechanisms and therapy. Nat. Rev. Neurol. 9, 106-118 (2013).

23. Jessen, F. et al. A conceptual framework for research on subjective cognitive decline in preclinical Alzheimer's disease. Alzheimers. Dement. 10, 844-52 (2014).

24. Rabin, L. A. et al. Subjective Cognitive Decline in Older Adults: An Overview of Self-Report Measures Used Across 19 International Research Studies. J. Alzheimers. Dis. 48 Suppl 1, S6386 (2015).

25. Jansen, W. J. et al. Prevalence of Cerebral Amyloid Pathology in Persons Without Dementia: A Meta-analysis. Jama 313, 1924-1938 (2015).

26. Villemagne, V. L. et al. Amyloid $\beta$ deposition, neurodegeneration, and cognitive decline in sporadic Alzheimer's disease: a prospective cohort study. Lancet Neurol. 12, 357-367 (2013).

27. Jicha, G. A. et al. Neuropathologic Outcome of Mild Cognitive Impairment Following Progression to Clinical Dementia. Arch. Neurol. 63, 674 (2006).

28. Serrano, C. M., Dillon, C., Leis, A., Taragano, F. E. \& Allegri, R. F. Mild cognitive Impairment: Risk of Dementia according to Subtypes. Actas españolas Psiquiatr. 41, 330-9 (2013).

29. Petersen, R. C. Mild cognitive impairment as a clinical entity and treatment target. Arch. Neurol. 62, 1160-1163; discussion 1167 (2004).

30. Palop, J. \& Mucke, L. Amyloid-beta Induced NeuronalDisease : From Synapses toward Neural Networks. Nat. Neurosci. 13, 812-818 (2010).

31. Clifford, R. J. J. et al. Update on hypothetical model of Alzheimer's disease biomarkers. Lancet Neurol. 12, 207-216 (2013).

32. de Leon, M. J. et al. Contribution of structural neuroimaging to the early diagnosis of Alzheimer's disease. Int Psychogeriatr 9 Suppl 1, 152-183 (1997).

33. Haan, W. De, Straaten, E. C. W. Van, Gouw, A. A. \& Stam, C. J. Altering neuronal excitability to preserve network connectivity in a computational model of Alzheimer' s disease. PLoS Comput. Biol. 1-23 (2017). doi:10.1371/journal.pcbi.1005707

34. Jones, D. T. et al. Cascading network failure across the Alzheimer's disease spectrum. Brain 139, 547-562 (2016).

35. Verdoorn, T. a et al. Evaluation and tracking of Alzheimer's disease severity using restingstate magnetoencephalography. J. Alzheimers. Dis. 26 Suppl 3, 239-55 (2011).

36. Engels, M. M. A. et al. Slowing of Hippocampal Activity Correlates with Cognitive Decline in Early Onset Alzheimer's Disease. An MEG Study with Virtual Electrodes. Front. Hum. Neurosci. 10, 238 (2016).

37. López, M. E. et al. MEG spectral analysis in subtypes of mild cognitive impairment. Age (Omaha). 36, 1095-1112 (2014).

38. Garcés, P. et al. Brain-wide slowing of spontaneous alpha rhythms in mild cognitive impairment. Front. Aging Neurosci. 5, 1-7 (2013).

39. Fernández, A. et al. MEG delta mapping along the healthy aging-Alzheimer's disease continuum: diagnostic implications. J. Alzheimers. Dis. 35, 495-507 (2013).

40. Berendse, H. ., Verbunt, J. P. ., Scheltens, P., van Dijk, B. . \& Jonkman, E. . Magnetoencephalographic analysis of cortical activity in Alzheimer's disease: a pilot study. Clin. Neurophysiol. 111, 604-612 (2000).

41. Osipova, D. et al. Source estimation of spontaneous MEG oscillations in mild cognitive impairment. Neurosci. Lett. 405, 57-61 (2006).

42. Locatelli, T., Cursi, M., Liberati, D., Franceschi, M. \& Comi, G. EEG coherence in Alzheimer's disease. Electroencephalogr. Clin. Neurophysiol. 106, 229-37 (1998).

43. Berendse, H. W., Verbunt, J. P., Scheltens, P., van Dijk, B. W. \& Jonkman, E. J. 
Magnetoencephalographic analysis of cortical activity in Alzheimer's disease: a pilot study. Clin. Neurophysiol. 111, 604-12 (2000).

44. Stam, C. J. et al. Magnetoencephalographic evaluation of resting-state functional connectivity in Alzheimer's disease. Neuroimage 32, 1335-44 (2006).

45. Alonso, J. F. et al. MEG Connectivity Analysis in Patients with Alzheimer's Disease Using Cross Mutual Information and Spectral Coherence. Ann. Biomed. Eng. 39, 524-536 (2011).

46. Lopez, M. E. et al. Alpha-Band Hypersynchronization in Progressive Mild Cognitive Impairment: A Magnetoencephalography Study. J. Neurosci. 34, 14551-14559 (2014).

47. Francis, P. T., Palmer, a. M., Snape, M. \& Wilcock, G. K. The cholinergic hypothesis of Alzheimer's disease: a review of progress. J. Neurol. Neurosurg. Psychiatry 66, 137-147 (1999).

48. Bajo, R. et al. Scopolamine effects on functional brain connectivity: a pharmacological model of Alzheimer's disease. Sci Rep 5, 9748 (2015).

49. Buccafusco, J. J. The Revival of Scopolamine Reversal for the Assessment of CognitionEnhancing Drugs. Methods of Behavior Analysis in Neuroscience (2009).

50. Canuet, L. et al. Network Disruption and Cerebrospinal Fluid Amyloid-Beta and PhosphoTau Levels in Mild Cognitive Impairment. J. Neurosci. 35, 10325-10330 (2015).

51. Albert, M. S. et al. The diagnosis of mild cognitive impairment due to Alzheimer's disease: Recommendations from the National Institute on Aging-Alzheimer's Association workgroups on diagnostic guidelines for Alzheimer's disease. Alzheimer's Dement. 7, 270279 (2011).

52. Vlassenko, A. G., Benzinger, T. L. S. \& Morris, J. C. PET amyloid-beta imaging in preclinical Alzheimer's disease. Biochim. Biophys. Acta - Mol. Basis Dis. 1822, 370-379 (2012).

53. Andreasen, N., Sjögren, M. \& Blennow, K. CSF markers for Alzheimer's disease: total tau, phospho-tau and Abeta42. World J. Biol. Psychiatry 4, 147-55 (2003).

54. Taeho Kim, $1, *$ George S. Vidal, 1 Maja Djurisic, 1 Christopher M. William, 2 Michael E. Birnbaum, 3 \& K. Christopher Garcia, 3 Bradley T. Hyman, 2 Carla J. Shatz1. Human LilrB2 Is a b-Amyloid Receptor and Its Murine Homolog PirB Regulates Synaptic Plasticity in an Alzheimer's Model. Science (80-. ). 7901, 207-210 (2013).

55. Grandjean, J. et al. Early Alterations in Functional Connectivity and White Matter Structure in a Transgenic Mouse Model of Cerebral Amyloidosis. 34, 13780-13789 (2014).

56. Garcés, P. et al. The Default Mode Network is functionally and structurally disrupted in amnestic mild cognitive impairment - A bimodal MEG-DTI study. NeuroImage Clin. 6, 214221 (2014).

57. Klupp, E. et al. In Alzheimer's disease, hypometabolism in low-amyloid brain regions may be a functional consequence of pathologies in connected brain regions. Brain Connect. 4, 37183 (2014).

58. Pineda-Pardo, J. A. et al. White Matter Damage Disorganizes Brain Functional Networks in Amnestic Mild Cognitive Impairment. Brain Connect. (2014). doi:10.1089/brain.2013.0208

59. Cohen, D. Magnetoencephalography: evidence of magnetic fields produced by alpha-rhythm currents. Science 161, 784-6 (1968).

60. Brenner, D., Williamson, S. J. \& Kaufman, L. Visually evoked magnetic fields of the human brain. Science 190, 480-2 (1975).

61. Teyler, T. J., Cuffin, B. N. \& Cohen, D. The visual evoked magnetoencephalogram. Life Sci. 17, 683-91 (1975).

62. Hari, R., Aittoniemi, K., Järvinen, M. L., Katila, T. \& Varpula, T. Auditory evoked transient and sustained magnetic fields of the human brain. Localization of neural generators. Exp. brain Res. 40, 237-40 (1980).

63. Reite, M., Edrich, J., Zimmerman, J. T. \& Zimmerman, J. E. Human magnetic auditory evoked 
fields. Electroencephalogr. Clin. Neurophysiol. 45, 114-7 (1978).

64. Hari, R., Hämäläinen, M., Kaukoranta, E., Reinikainen, K. \& Teszner, D. Neuromagnetic responses from the second somatosensory cortex in man. Acta Neurol. Scand. 68, 207-12 (1983).

65. Salmelin, R., Hari, R., Lounasmaa, O. V. \& Sams, M. Dynamics of brain activation during picture naming. Nature 368, 463-465 (1994).

66. Hari, R. \& Salmelin, R. Human cortical oscillations: a neuromagnetic view through the skull. Trends Neurosci. 20, 44-49 (1997).

67. Tiihonen, J., Kajola, M. \& Hari, R. Magnetic mu rhythm in man. Neuroscience 32, 793-800 (1989).

68. Gray, C. M., König, P., Engel, A. K. \& Singer, W. Oscillatory responses in cat visual cortex exhibit inter-columnar synchronization which reflects global stimulus properties. Nature 338, 334-7 (1989).

69. Salenius, S. \& Hari, R. Synchronous cortical oscillatory activity during motor action. Curr. Opin. Neurobiol. 13, 678-84 (2003).

70. Fuentemilla, L., Penny, W. D., Cashdollar, N., Bunzeck, N. \& Düzel, E. Theta-coupled periodic replay in working memory. Curr. Biol. 20, 606-12 (2010).

71. DeFelipe, J. \& Ramón y Cajal, S. Cajal's butterflies of the soul : science and art. (Oxford University Press, 2010).

72. Buzsáki, G., Anastassiou, C. A. \& Koch, C. The origin of extracellular fields and currents--EEG, ECoG, LFP and spikes. Nat. Rev. Neurosci. 13, 407-20 (2012).

73. Lodish, H. F. Molecular cell biology. (W.H. Freeman, 2000).

74. Zeng, H. \& Sanes, J. R. Neuronal cell-type classification: challenges, opportunities and the path forward. Nat. Rev. Neurosci. 18, 530-546 (2017).

75. Spruston, N. Pyramidal neurons: dendritic structure and synaptic integration. Nat. Rev. Neurosci. 9, 206-221 (2008).

76. Stufflebeam, R. Neurons, Synapses, Action Potentials, and Neurotransmission - The Mind Project. Available at:

http://www.mind.ilstu.edu/curriculum/neurons_intro/neurons_intro.php. (Accessed: 15th October 2017)

77. Institute for Learning \& Brain Sciences. University of Washington. What is Magnetoencephalography (MEG)? | Institute for Learning and Brain Sciences (I-LABS). Available at: http://ilabs.washington.edu/what-magnetoencephalography-meg. (Accessed: 15th October 2017)

78. Hansen, P. C., Kringelbach, M. L. \& Salmelin, R. MEG: An Introduction to Methods. (Oxford University Press, 2010).

79. Hämäläinen, M., Hari, R., Ilmoniemi, R. J., Knuutila, J. \& Lounasmaa, O. V.

Magnetoencephalography-theory, instrumentation, and applications to noninvasive studies of the working human brain. Rev. Mod. Phys. 65, 413-497 (1993).

80. Hari, R. \& Salmelin, R. Magnetoencephalography: From SQUIDs to neuroscience. Neuroimage 20th Anniversary Special Edition. Neuroimage 61, 386-396 (2012).

81. Stephen Whitmarsh. The basics of MEG measurement and instrumentation [FieldTrip Toolbox video]. (2013). Available at: http://www.fieldtriptoolbox.org/video\#the_basics_of_meg_measurement_and_instrumenta tion. (Accessed: 4th October 2017)

82. Drung, D. . et al. Highly Sensitive and Easy-to-Use SQUID Sensors. IEEE Trans. Appl. Supercond. 17, 699-704 (2007).

83. Duffau, H. Brain Mapping. (2011). doi:10.1007/978-3-7091-0723-2 
84. Tudor, M., Tudor, L. \& Tudor, K. I. [Hans Berger (1873-1941)--the history of electroencephalography]. Acta Med. Croatica 59, 307-13 (2005).

85. Fernández, T. et al. EEG activation patterns during the performance of tasks involving different components of mental calculation. Electroencephalogr. Clin. Neurophysiol. 94, 17582 (1995).

86. Harmony, T. The functional significance of delta oscillations in cognitive processing. Front. Integr. Neurosci. 7, (2013).

87. Harmony, T. et al. EEG delta activity: An indicator of attention to internal processing during performance of mental tasks. Int. J. Psychophysiol. 24, 161-171 (1996).

88. Klimesch, W. EEG alpha and theta oscillations reflect cognitive and memory performance: A review and analysis. Brain Research Reviews 29, 169-195 (1999).

89. Sederberg, P. B., Kahana, M. J., Howard, M. W., Donner, E. J. \& Madsen, J. R. Theta and gamma oscillations during encoding predict subsequent recall. J. Neurosci. 23, 10809-14 (2003).

90. Kahana, M. J. The Cognitive Correlates of Human Brain Oscillations. J. Neurosci. 26, 16691672 (2006).

91. Khader, P. H., Jost, K., Ranganath, C. \& Rösler, F. Theta and alpha oscillations during workingmemory maintenance predict successful long-term memory encoding. Neurosci. Lett. 468, 339-343 (2010).

92. Klimesch, W. $\alpha$-band oscillations, attention, and controlled access to stored information. Trends Cogn. Sci. 16, 606-17 (2012).

93. Buschman, T. J. \& Miller, E. K. Top-Down Versus Bottom-Up Control of Attention in the Prefrontal and Posterior Parietal Cortices. Science (80-. ). 315, 1860-1862 (2007).

94. Engel, A. K. \& Fries, P. Beta-band oscillations-signalling the status quo? Curr. Opin. Neurobiol. 20, 156-165 (2010).

95. Jensen, O., Kaiser, J. \& Lachaux, J.-P. Human gamma-frequency oscillations associated with attention and memory. Trends Neurosci. 30, 317-324 (2007).

96. Elekta-Neuromag. MaxFilter 2.2 User's Guide. October (2010).

97. Elekta-Neuromag. Elekta Neuromag $®$ Systems. Service Manual. (2014).

98. Taulu, S. \& Kajola, M. Presentation of electromagnetic multichannel data: The signal space separation method. J. Appl. Phys. 97, 124905 (2005).

99. Taulu, S., Simola, J. \& Kajola, M. Applications of the signal space separation method. IEEE Trans. Signal Process. 53, 3359-3372 (2005).

100. Taulu, S. \& Simola, J. Spatiotemporal signal space separation method for rejecting nearby interference in MEG measurements. Phys. Med. Biol. 51, 1759-1768 (2006).

101. Tesche, C. D. et al. Signal-space projections of MEG data characterize both distributed and well-localized neuronal sources. Electroencephalogr. Clin. Neurophysiol. 95, 189-200 (1995).

102. Uusitalo, M. A. \& Ilmoniemi, R. J. Signal-space projection method for separating MEG or EEG into components. Med. Biol. Eng. Comput. 35, 135-40 (1997).

103. Langlois, D., Chartier, S. \& Gosselin, D. An Introduction to Independent Component Analysis: InfoMax and FastICA algorithms. Tutor. Quant. Methods Psychol. 6, 31-38 (2010).

104. Vigário, R., Jousmäki, V., Hämäläinen, M., Hari, R. \& Oja, E. Independent Component Analysis for Identification of Artifacts in Magnetoencephalographic Recordings. Adv. Neural Inf. Process. Syst. 229-235 (1998). doi:10.1109/10.841330

105. Hyvärinen, A. \& Oja, E. Independent component analysis: Algorithms and applications. Neural Networks 13, 411-430 (2000).

106. Mosher, J. C., Leahy, R. M. \& Lewis, P. S. EEG and MEG: forward solutions for inverse methods. IEEE Trans. Biomed. Eng. 46, 245-259 (1999). 
107. Baillet, S., Mosher, J. C. \& Leahy, R. M. Electromagnetic brain mapping. IEEE Signal Process. Mag. 18, 14-30 (2001).

108. Henson, R. N., Mattout, J., Phillips, C. \& Friston, K. J. Selecting forward models for MEG source-reconstruction using model-evidence. Neuroimage 46, 168-76 (2009).

109. Thevenet, M., Bertrand, O., Perrin, F., Dumont, T. \& Pernier, J. The finite element method for a realistic head model of electrical brain activities: preliminary results. Clin. Phys. Physiol. Meas. 12, 89-94 (1991).

110. Van Uitert, R. \& Johnson, C. Can a spherical model substitute for a realistic model in forward and inverse MEG simulations. Proc. BIOMAG 25, 52-68 (2002).

111. Sarvas, J. Basic mathematical and electromagnetic concepts of the biomagnetic inverse problem. Phys. Med. Biol. 32, 11-22 (1987).

112. Huang, M. X., Mosher, J. C. \& Leahy, R. M. A sensor-weighted overlapping-sphere head model and exhaustive head model comparison for MEG. Phys Med Biol 44, 423-440 (1999).

113. Fuchs, M., Drenckhahn, R., Wischmann, H. \& Wagner, M. An improved boundary element method for realistic volume-conductor modeling. IEEE Trans. Biomed. Eng. 45, 980-997 (1998).

114. Bertero, M., Mol, C. De \& Pike, E. R. Linear inverse problems with discrete data. I. General formulation and singular system analysis. Inverse Probl. 1, 301-330 (1985).

115. Golub, G. H. (Gene H. \& Van Loan, C. F. Matrix computations. (Johns Hopkins University Press, 1996).

116. Hauk, O., Wakeman, D. G. \& Henson, R. Comparison of noise-normalized minimum norm estimates for MEG analysis using multiple resolution metrics. Neuroimage 54, 1966-74 (2011).

117. Hämäläinen, M. S. \& Ilmoniemi, R. J. Interpreting magnetic fields of the brain: minimum norm estimates. Med. Biol. Eng. Comput. 32, 35-42 (1994).

118. Lin, F.-H. et al. Assessing and improving the spatial accuracy in MEG source localization by depth-weighted minimum-norm estimates. Neuroimage 31, 160-171 (2006).

119. Hillebrand, A. \& Barnes, G. R. Beamformer Analysis of MEG Data. Int. Rev. Neurobiol. 68, 149-171 (2005).

120. Maestú, F., Pereda, E. \& del Pozo, F. CONECTIVIDAD FUNCIONAL Y ANATÓMICA EN EL CEREBRO HUMANO. (Elsevier, 2015).

121. Pereda, E., Quiroga, R. Q. \& Bhattacharya, J. Nonlinear multivariate analysis of neurophysiological signals. Prog. Neurobiol. 77, 1-37 (2005).

122. Niso, G. et al. HERMES: Towards an Integrated Toolbox to Characterize Functional and Effective Brain Connectivity. Neuroinformatics 11, 405-434 (2013).

123. Bastos, A. M. \& Schoffelen, J.-M. A Tutorial Review of Functional Connectivity Analysis Methods and Their Interpretational Pitfalls. Front. Syst. Neurosci. 9, (2016).

124. Rodriguez, E. et al. Perception's shadow: long-distance synchronization of human brain activity. Nature 397, 430-433 (1999).

125. Varela, F., Lachaux, J.-P., Rodriguez, E. \& Martinerie, J. The brainweb: phase synchronization and large-scale integration. Nat. Rev. Neurosci. 2, 229-239 (2001).

126. Lachaux, J. P., Rodriguez, E., Martinerie, J. \& Varela, F. J. Measuring phase synchrony in brain signals. Hum. Brain Mapp. 8, 194-208 (1999).

127. Osipova, D. et al. Effects of scopolamine on MEG spectral power and coherence in elderly subjects. Clin. Neurophysiol. 114, 1902-7 (2003).

128. Tzourio-Mazoyer, N. et al. Automated Anatomical Labeling of Activations in SPM Using a Macroscopic Anatomical Parcellation of the MNI MRI Single-Subject Brain. Neuroimage 15, 273-289 (2002). 
129. Tadel, F., Baillet, S., Mosher, J. C., Pantazis, D. \& Leahy, R. M. Brainstorm: a user-friendly application for MEG/EEG analysis. Comput. Intell. Neurosci. 2011, 879716 (2011).

130. Holmes, C. J. et al. Enhancement of MR images using registration for signal averaging. J. Comput. Assist. Tomogr. 22, 324-33

131. Mosher, J. C., Baillet, S. \& Leahy, R. M. EQUIVALENCE OF LINEAR APPROACHES IN BIOELECTROMAGNETIC INVERSE SOLUTIONS. IEEE Work. Stat. Signal Process. 294-297 (2003).

132. van Straaten, E. C. W. \& Stam, C. J. Structure out of chaos: Functional brain network analysis with EEG, MEG, and functional MRI. Eur. Neuropsychopharmacol. 23, 7-18 (2013).

133. Rubinov, M. \& Sporns, O. Complex network measures of brain connectivity: Uses and interpretations. Neuroimage 52, 1059-1069 (2010).

134. Sporns, O. Network attributes for segregation and integration in the human brain. Curr. Opin. Neurobiol. 23, 162-171 (2013).

135. Sporns, 0. Contributions and challenges for network models in cognitive neuroscience. Nat. Neurosci. 17, 652-660 (2014).

136. Stam, C. J. et al. Graph theoretical analysis of magnetoencephalographic functional connectivity in Alzheimer's disease. Brain 132, 213-24 (2009).

137. Bajo, R. et al. Functional connectivity in mild cognitive impairment during a memory task: implications for the disconnection hypothesis. J. Alzheimers. Dis. 22, 183-93 (2010).

138. Cj, S., Y, V. D. M., Yal, P. \& Eeg, S. P. EEG synchronization in mild cognitive impairment and Alzheimer' s disease. Acta Neurol. Scand. 108, 90-96 (2003).

139. Pijnenburg, Y. a L. et al. EEG synchronization likelihood in mild cognitive impairment and Alzheimer's disease during a working memory task. Clin. Neurophysiol. 115, 1332-9 (2004)

140. Jeong, J. EEG dynamics in patients with Alzheimer's disease. Clin. Neurophysiol. 115, 1490505 (2004).

141. Babiloni, C. et al. Mapping distributed sources of cortical rhythms in mild Alzheimer's disease. A multicentric EEG study. Neuroimage 22, 57-67 (2004).

142. Koenig, T. et al. Decreased EEG synchronization in Alzheimer's disease and mild cognitive impairment. Neurobiol. Aging 26, 165-71 (2005).

143. Sperling, R. A. et al. Functional Alterations in Memory Networks in Early Alzheimer's Disease. NeuroMolecular Med. 12, 27-43 (2010).

144. Iturria-Medina, Y. et al. Characterizing brain anatomical connections using diffusion weighted MRI and graph theory. Neuroimage 36, 645-660 (2007).

145. Reis, P. M. R., Eckhardt, H., Denise, P., Bodem, F. \& Lochmann, M. Localization of scopolamine induced electrocortical brain activity changes, in healthy humans at rest. J. Clin. Pharmacol. 53, 619-625 (2013).

146. Iturria-Medina, Y., Sotero, R. C., Canales-Rodríguez, E. J., Alemán-Gómez, Y. \& Melie-García, L. Studying the human brain anatomical network via diffusion-weighted MRI and Graph Theory. Neuroimage 40, 1064-1076 (2008).

147. Wink, A. M., Bernard, F., Salvador, R., Bullmore, E. \& Suckling, J. Age and cholinergic effects on hemodynamics and functional coherence of human hippocampus. Neurobiol. Aging 27, 1395-404 (2006).

148. Brier, M. R. et al. Loss of intranetwork and internetwork resting state functional connections with Alzheimer's disease progression. J. Neurosci. 32, 8890-9 (2012).

149. Zaidel, L. et al. Donepezil effects on hippocampal and prefrontal functional connectivity in Alzheimer's disease: preliminary report. J. Alzheimers. Dis. 31 Suppl 3, S221-6 (2012).

150. Berendse, H. W., Verbunt, J. P., Scheltens, P., van Dijk, B. W. \& Jonkman, E. J.

Magnetoencephalographic analysis of cortical activity in Alzheimer's disease: a pilot study. 
Clin. Neurophysiol. 111, 604-12 (2000).

151. Albert, M. S. et al. The diagnosis of mild cognitive impairment due to Alzheimer's disease: Recommendations from the National Institute on Aging-Alzheimer's Association workgroups on diagnostic guidelines for Alzheimer's disease. Alzheimer's Dement. 7, 270279 (2011).

152. Mattsson, N. et al. CSF biomarker variability in the Alzheimer's Association quality control program. Alzheimer's Dement. 9, 251-261 (2013).

153. Hipp, J. F., Hawellek, D. J., Corbetta, M., Siegel, M. \& Engel, A. K. Large-scale cortical correlation structure of spontaneous oscillatory activity. Nat. Neurosci. 15, 884-890 (2012).

154. Hua, K. et al. Tract probability maps in stereotaxic spaces: Analyses of white matter anatomy and tract-specific quantification. Neuroimage 39, 336-347 (2008).

155. van Rossum, I. A. et al. Injury markers but not amyloid markers are associated with rapid progression from mild cognitive impairment to dementia in Alzheimer's disease. J. Alzheimers. Dis. 29, 319-27 (2012).

156. Khan, U. A. et al. Molecular drivers and cortical spread of lateral entorhinal cortex dysfunction in preclinical Alzheimer's disease. Nat. Neurosci. 17, 304-311 (2013).

157. Serrano-Pozo, A., Frosch, M. P., Masliah, E. \& Hyman, B. T. Neuropathological Alterations in Alzheimer Disease. Cold Spring Harb. Perspect. Med. 1, a006189-a006189 (2011).

158. Spires-Jones, T. L. \& Hyman, B. The Intersection of Amyloid Beta and Tau at Synapses in Alzheimer's Disease. Neuron 82, 756-771 (2014).

159. Amlien, I. K. et al. Mild Cognitive Impairment: Cerebrospinal Fluid Tau Biomarker Pathologic Levels and Longitudinal Changes in White Matter Integrity. Radiology 266, 295-303 (2013).

160. Sheline, Y. I. et al. Amyloid Plaques Disrupt Resting State Default Mode Network Connectivity in Cognitively Normal Elderly. Biol. Psychiatry 67, 584-587 (2010).

161. Li, R. et al. Alterations of directional connectivity among resting-state networks in Alzheimer disease. AJNR. Am. J. Neuroradiol. 34, 340-5 (2013).

162. Drzezga, A. et al. Neuronal dysfunction and disconnection of cortical hubs in non-demented subjects with elevated amyloid burden. Brain 134, 1635-1646 (2011).

163. Carbonell, F. et al. Hierarchical Multivariate Covariance Analysis of Metabolic Connectivity. J. Cereb. Blood Flow Metab. 34, 1936-1943 (2014).

164. Campo, P. et al. Remote Effects of Hippocampal Sclerosis on Effective Connectivity during Working Memory Encoding: A Case of Connectional Diaschisis? Cereb. Cortex 22, 1225$1236(2012)$.

165. Wang, L. et al. Cerebrospinal Fluid A $\beta 42$, Phosphorylated Tau 181 , and Resting-State Functional Connectivity. JAMA Neurol. 70, 1242-8 (2013).

166. Grady, C. L. et al. Evidence from functional neuroimaging of a compensatory prefrontal network in Alzheimer's disease. J. Neurosci. 23, 986-93 (2003).

167. McKhann, G. M. et al. The diagnosis of dementia due to Alzheimer's disease: recommendations from the National Institute on Aging-Alzheimer's Association workgroups on diagnostic guidelines for Alzheimer's disease. Alzheimers. Dement. 7, 263-9 (2011).

168. Jack, C. R. et al. Introduction to the recommendations from the National Institute on AgingAlzheimer's Association workgroups on diagnostic guidelines for Alzheimer's disease. Alzheimers. Dement. 7, 257-62 (2011).

169. Jack, C. R. et al. Rates of $\beta$-amyloid accumulation are independent of hippocampal neurodegeneration. Neurology 82, 1605-12 (2014).

170. Shah, Y., Tangalos, E. G. \& Petersen, R. C. Mild cognitive impairment. When is it a precursor to Alzheimer's disease? Geriatrics 55, 62, 65-8 (2000). 
171. Farias, S., Mungas, D. \& Jagust, W. Degree of discrepancy between self and other-reported everyday functioning by cognitive status: dementia, mild cognitive impairment, and healthy elders. J. Alzheimers. Dis. 9, 827-834 (2005).

172. Albert M, Blacker D, Moss MB, Tanzi R, M. J. Longitudinal change in cognitive performance among individuals with mild cognitive impairment. Neuropsychology 21, 158-69. (2007).

173. Gomar, J. J. et al. Utility of combninations of biomarkers, cognitive markers, and risk factors to predict convresion from Mild cognitive impairment to Alzheimer disease in patients in the Alzheimer's disease neuroimaging initiative. Arch Gen Psychiatry 68, 961-969 (2011).

174. Eskildsen, S. F. et al. Prediction of Alzheimer's disease in subjects with mild cognitive impairment from the ADNI cohort using patterns of cortical thinning. Neuroimage $\mathbf{6 5}, 511-$ 21 (2013).

175. Liu, Y. et al. Impaired Long Distance Functional Connectivity and Weighted Network Architecture in Alzheimer's Disease. Cereb. Cortex (2013). doi:10.1093/cercor/bhs410

176. Hatashita, S. \& Yamasaki, H. Diagnosed Mild Cognitive Impairment Due to Alzheimer's Disease with PET Biomarkers of Beta Amyloid and Neuronal Dysfunction. PLoS One 8, e66877 (2013).

177. Grimmer, T. et al. The usefulness of amyloid imaging in predicting the clinical outcome after two years in subjects with mild cognitive impairment. Curr. Alzheimer Res. 10, 82-5 (2013).

178. Riemenschneider, M. et al. Cerebrospinal Fluid Tau and Beta-Amyloid 42 Proteins Identify Alzheimer Disease in Subjects With Mild Cognitive Impairment. Arch Neurol 59, 1729-1734 (2002).

179. Hampel, H. et al. Value of CSF beta-amyloid1-42 and tau as predictors of Alzheimer's disease in patients with mild cognitive impairment. Mol. Psychiatry 9, 705-10 (2004).

180. Oldfield, R. C. The assessment and analysis of handedness: the Edinburgh inventory. Neuropsychologia 9, 97-113 (1971).

181. López, M. E. et al. Searching for Primary Predictors of Conversion from Mild Cognitive Impairment to Alzheimer's Disease: A Multivariate Follow-Up Study. J. Alzheimer's Dis. 52, 133-143 (2016).

182. Bai, Y. et al. A prospective, randomized, single-blinded trial on the effect of early rehabilitation on daily activities and motor function of patients with hemorrhagic stroke. J. Clin. Neurosci. 19, 1376-1379 (2012).

183. Oostenveld, R., Fries, P., Maris, E. \& Schoffelen, J.-M. FieldTrip: Open Source Software for Advanced Analysis of MEG, EEG, and Invasive Electrophysiological Data. Comput. Intell. Neurosci. 2011, 1-9 (2011).

184. Van Veen, B. D., Van Drongelen, W., Yuchtman, M. \& Suzuki, A. Localization of brain electrical activity via linearly constrained minimum variance spatial filtering. IEEE Trans. Biomed. Eng. 44, 867-880 (1997).

185. Lobier, M., Siebenhühner, F., Palva, S. \& Palva, J. M. Phase transfer entropy: A novel phasebased measure for directed connectivity in networks coupled by oscillatory interactions. Neuroimage 85, 853-872 (2014).

186. Schreiber, T. Measuring information transfer. Phys. Rev. Lett. 85, 461-464 (2000).

187. Hillebrand, A. et al. Direction of information flow in large-scale resting-state networks is frequency-dependent. Proc. Natl. Acad. Sci. 113, 3867-3872 (2016).

188. Stam, C. J. et al. Graph theoretical analysis of magnetoencephalographic functional connectivity in Alzheimer's disease. Brain 132, 213-224 (2009).

189. Sanz-Arigita, E. J. et al. Loss of 'small-world' networks in Alzheimer's disease: graph analysis of FMRI resting-state functional connectivity. PLoS One 5, e13788 (2010).

190. Brier, M. R. et al. Functional connectivity and graph theory in preclinical Alzheimer's disease. Neurobiol. Aging 35, 757-768 (2014). 
191. He, Y., Chen, Z., Gong, G. \& Evans, A. Neuronal Networks in Alzheimer's Disease. 333-350 (2009).

192. Guimerà, R. \& Nunes Amaral, L. A. Functional cartography of complex metabolic networks. Nature 433, 895-900 (2005).

193. Power, J. D., Schlaggar, B. L., Lessov-Schlaggar, C. N. \& Petersen, S. E. Evidence for hubs in human functional brain networks. Neuron 79, 798-813 (2013).

194. Battiston, F., Nicosia, V. \& Latora, V. Structural measures for multiplex networks. Phys. Rev. E 89, 32804 (2014).

195. De Vico Fallani, F., Latora, V. \& Chavez, M. A Topological Criterion for Filtering Information in Complex Brain Networks. PLOS Comput. Biol. 13, e1005305 (2017).

196. Guillon, J. et al. Loss of brain inter-frequency hubs in Alzheimer's disease. (2016).

197. Esfahlani, F. Z. \& Sayama, H. A Percolation-based Thresholding Method with Applications in Functional Connectivity Analysis. (2017).

198. van Wijk, B. C. M., Stam, C. J. \& Daffertshofer, A. Comparing Brain Networks of Different Size and Connectivity Density Using Graph Theory. PLoS One 5, e13701 (2010).

199. Fornito, A., Zalesky, A. \& Breakspear, M. Graph analysis of the human connectome: Promise, progress, and pitfalls. Neuroimage 80, 426-444 (2013).

200. Dimitriadis, S. I., Salis, C., Tarnanas, I. \& Linden, D. E. Topological Filtering of Dynamic Functional Brain Networks Unfolds Informative Chronnectomics: A Novel Data-Driven Thresholding Scheme Based on Orthogonal Minimal Spanning Trees (OMSTs). Front. Neuroinform. 11, (2017).

201. Stam, C. J. et al. The trees and the forest: Characterization of complex brain networks with minimum spanning trees. Int. J. Psychophysiol. 92, 129-138 (2014).

202. Maris, E. \& Oostenveld, R. Nonparametric statistical testing of EEG- and MEG-data. J. Neurosci. Methods 164, 177-90 (2007).

203. Stam, C. J. Modern network science of neurological disorders. Nat. Rev. Neurosci. 15, 683-95 (2014).

204. Kearns, M. \& Ron, D. Algorithmic Stability and Sanity-Check Bounds for Leave-One-Out Cross-Validation. (1997).

205. Cawley, G. C. \& Talbot, N. L. C. EEcient leave-one-out cross-validation of kernel Fisher discriminant classiÿers. Pattern Recognit. 36, 2585-2592 (2003).

206. Clopper, C. J. \& Pearson, E. S. The Use of Confidence or Fiducial Limits Illustrated in the Case of the Binomial. Biometrika 26, 404 (1934).

207. Prichep, L. Quantitative EEG and Electromagnetic brain imaging in aging and in the evolution of dementia.pdf. (2007).

208. Prichep, L. S. et al. Prediction of longitudinal cognitive decline in normal elderly with subjective complaints using electrophysiological imaging. Neurobiol. Aging 27, 471-481 (2006).

209. Modrego, P. J. Predictors of conversion to dementia of probable Alzheimer type in patients with mild cognitive impairment. Curr. Alzheimer Res. 3, 161-70 (2006).

210. Antila, K. et al. The PredictAD project : development of novel biomarkers and analysis software for early diagnosis of the Alzheimer $\hat{a} €^{\mathrm{TM}} \mathrm{s}$ disease The PredictAD project : development of novel biomarkers and analysis software for early diagnosis of the Alzheimer 's disease. (2013).

211. Wang, J. et al. Disrupted functional brain connectome in individuals at risk for Alzheimer's disease. Biol. Psychiatry 73, 472-81 (2013).

212. Wang, R. et al. Decreased coherence and functional connectivity of electroencephalograph in Alzheimer â€TM s disease Decreased coherence and functional connectivity of 
electroencephalograph in Alzheimer's disease. 33136, (2015).

213. Zhou, B. et al. Aberrant Functional Connectivity Architecture in Alzheimer's Disease and Mild Cognitive Impairment: A Whole-Brain, Data-Driven Analysis. Biomed Res. Int. 2015, 19 (2015).

214. Engels, M. M. et al. Declining functional connectivity and changing hub locations in Alzheimer's disease: an EEG study. BMC Neurol. 15, 145 (2015).

215. Güntekin, B., Saatçi, E. \& Yener, G. Decrease of evoked delta, theta and alpha coherences in Alzheimer patients during a visual oddball paradigm. Brain Res. 1235, 109-116 (2008).

216. Deiber, M.-P. et al. Abnormal-induced theta activity supports early directed-attention network deficits in progressive MCI. Neurobiol. Aging 30,1444-52 (2009).

217. Missonnier, P. et al. Early disturbances of gamma band dynamics in mild cognitive impairment. J. Neural Transm. 117, 489-498 (2010).

218. Raghavachari, S. et al. Gating of human theta oscillations by a working memory task. J. Neurosci. 21, 3175-83 (2001).

219. Gevins, A., Smith, M. E., McEvoy, L. \& Yu, D. High-resolution EEG mapping of cortical activation related to working memory: effects of task difficulty, type of processing, and practice. Cereb. Cortex 7, 374-85 (1997).

220. Thompson, P. M. et al. Dynamics of gray matter loss in Alzheimer's disease. J. Neurosci. 23, 994-1005 (2003).

221. Montez, T. et al. Altered temporal correlations in parietal alpha and prefrontal theta oscillations in early-stage Alzheimer disease. Proc. Natl. Acad. Sci. 106, 1614-1619 (2009).

222. Park, Y. M. et al. Decreased EEG synchronization and its correlation with symptom severity in Alzheimer's disease. Neurosci. Res. 62, 112-117 (2008).

223. Sarnthein, J., Petsche, H., Rappelsberger, P., Shaw, G. L. \& von Stein, A. Synchronization between prefrontal and posterior association cortex during human working memory. Proc. Natl. Acad. Sci. U. S. A. 95, 7092-6 (1998).

224. Anokhin, A. P., Lutzenberger, W. \& Birbaumer, N. Spatiotemporal organization of brain dynamics and intelligence: an EEG study in adolescents. Int. J. Psychophysiol. 33, 259-73 (1999).

225. Stam, C. J. Brain dynamics in theta and alpha frequency bands and working memory performance in humans. Neurosci. Lett. 286, 115-8 (2000).

226. Carrera, E. \& Tononi, G. Diaschisis: past, present, future. Brain 137, 2408-2422 (2014).

227. McKee, A. C. et al. Visual association pathology in preclinical Alzheimer disease. J. Neuropathol. Exp. Neurol. 65, 621-30 (2006).

228. Aine, C. J. et al. Aging: compensation or maturation? Neuroimage 32, 1891-904 (2006).

229. Amanzio, M. et al. Unawareness of deficits in Alzheimer's disease: role of the cingulate cortex. Brain 134, 1061-76 (2011).

230. Stam, C. J., van der Made, Y., Pijnenburg, Y. A. L. \& Scheltens, P. EEG synchronization in mild cognitive impairment and Alzheimer's disease. Acta Neurol. Scand. 108, 90-6 (2003).

231. Koelewijn, L. et al. Alzheimer's disease disrupts alpha and beta-band resting-state oscillatory network connectivity. (2017). doi:10.1016/j.clinph.2017.04.018

232. Liang, P., Wang, Z., Yang, Y., Jia, X. \& Li, K. Functional disconnection and compensation in mild cognitive impairment: evidence from DLPFC connectivity using resting-state fMRI. PLoS One 6, e22153 (2011).

233. Abuhassan, K., Coyle, D., Belatreche, A. \& Maguire, L. Compensating for synaptic loss in Alzheimer's disease. J. Comput. Neurosci. 36, 19-37 (2014).

234. Gallego-Jutglà, E., Solé-Casals, J., Vialatte, F.-B., Dauwels, J. \& Cichocki, A. A Theta-Band EEG Based Index for Early Diagnosis of Alzheimer's Disease. J. Alzheimers. Dis. 43, 1175-1184 
(2014).

235. Poil, S. S. et al. Integrative EEG biomarkers predict progression to Alzheimer's disease at the MCI stage. Front. Aging Neurosci. 5, 1-12 (2013).

236. Al-Jumeily, D., Iram, S., Vialatte, F.-B., Fergus, P. \& Hussain, A. A Novel Method of Early Diagnosis of Alzheimer's Disease Based on EEG Signals. Sci. World J. 2015, 1-11 (2015).

237. Buckner, R. L. \& Louis, S. Memory and Executive Function Review in Aging and AD : Multiple Factors that Cause Decline and Reserve Factors that Compensate. Neuron 44, 195-208 (2004).

238. Albert, R., Jeong, H. \& Barabási, A.-L. Error and attack tolerance of complex networks. Nature 406, 378-382 (2000).

239. Jeong, H., Mason, S. P., Barabási, A.-L. \& Oltvai, Z. N. Lethality and centrality in protein networks. Nature 411, 41-42 (2001).

240. Hsiao, F.-J., Wang, Y.-J., Yan, S.-H., Chen, W.-T. \& Lin, Y.-Y. Altered oscillation and synchronization of default-mode network activity in mild Alzheimer's disease compared to mild cognitive impairment: an electrophysiological study. PLoS One 8, e68792 (2013).

241. Engels, M. M. A. et al. Directional information flow in patients with Alzheimer's disease. A source-space resting-state MEG study. NeuroImage Clin. 15, 673-681 (2017).

242. Juan-Cruz, C., Gómez, C., Poza, J., Fernández, A. \& Hornero, R. Assessment of Effective Connectivity in Alzheimer's Disease Using Granger Causality. in 763-767 (Springer, Cham, 2017). doi:10.1007/978-3-319-46669-9_125

243. Vecchio, F. \& Babiloni, C. Direction of Information Flow in Alzheimer's Disease and MCI Patients. Int. J. Alzheimers. Dis. 2011, 214580 (2011).

244. Stam, C. J. et al. Generalized synchronization of MEG recordings in Alzheimer's Disease: evidence for involvement of the gamma band. J. Clin. Neurophysiol. 19, 562-74 (2002).

245. Qi, Z. et al. Impairment and compensation coexist in amnestic MCI default mode network. Neuroimage 50, 48-55 (2010).

246. Buldyrev, S. V., Parshani, R., Paul, G., Stanley, H. E. \& Havlin, S. Catastrophic cascade of failures in interdependent networks. Nature 464, 1025-1028 (2010).

247. Yu, M. et al. Selective impairment of hippocampus and posterior hub areas in Alzheimer?s disease: an MEG-based multiplex network study. Brain 140, 1466-1485 (2017).

248. Mevel, K., Chételat, G., Eustache, F. \& Desgranges, B. The default mode network in healthy aging and Alzheimer's disease. Int. J. Alzheimers. Dis. 2011, 535816 (2011).

249. de Haan, W. et al. Disrupted modular brain dynamics reflect cognitive dysfunction in Alzheimer's disease. Neuroimage 59, 3085-93 (2012).

250. Stam, C. J., Nolte, G. \& Daffertshofer, A. Phase lag index: Assessment of functional connectivity from multi channel EEG and MEG with diminished bias from common sources. Hum. Brain Mapp. 28, 1178-1193 (2007).

251. Colclough, G. L. et al. How reliable are MEG resting-state connectivity metrics? Neuroimage 138, 284-293 (2016).

252. Colclough, G. L., Brookes, M. J., Smith, S. M. \& Woolrich, M. W. A symmetric multivariate leakage correction for MEG connectomes. Neuroimage 117, 439-448 (2015).

253. Brookes, M. J., Woolrich, M. W. \& Barnes, G. R. Measuring functional connectivity in MEG: A multivariate approach insensitive to linear source leakage. Neuroimage $63,910-920$ (2012).

254. Wens, V. et al. A geometric correction scheme for spatial leakage effects in MEG/EEG seedbased functional connectivity mapping. Hum. Brain Mapp. 36, 4604-4621 (2015).

255. Bruña, R., Maestú, F. \& Pereda, E. Phase Locking Value revisited: teaching new tricks to an old dog. (2017). 
256. Garcés, P., Martín-Buro, M. C. \& Maestú, F. Quantifying the Test-Retest Reliability of Magnetoencephalography Resting-State Functional Connectivity. Brain Connect. 6, 448-460 (2016).

257. Finger, H. et al. Modeling of Large-Scale Functional Brain Networks Based on Structural Connectivity from DTI: Comparison with EEG Derived Phase Coupling Networks and Evaluation of Alternative Methods along the Modeling Path. PLoS Comput. Biol. 12, 1-28 (2016).

258. Vicente, R., Gollo, L. L., Mirasso, C. R., Fischer, I. \& Pipa, G. Dynamical relaying can yield zero time lag neuronal synchrony despite long conduction delays. Proc. Natl. Acad. Sci. 105, 17157-17162 (2008).

259. Gollo, L. L., Mirasso, C. \& Villa, A. E. P. Dynamic control for synchronization of separated cortical areas through thalamic relay. Neuroimage 52, 947-955 (2010).

260. Mandke, K. et al. Comparing multilayer brain networks between groups: Introducing graph metrics and recommendations. Neuroimage 166, 371-384 (2018). 